\title{
Idempotentes Primitivos em Álgebras de Grupo Finitas e Códigos Minimais
}

\author{
Rafael Budaibes \\ TESE APRESENTADA \\ $\mathrm{AO}$ \\ Instituto DE Matemática e Estatística \\ DA \\ Universidade DE SÃo Paulo \\ PARA \\ OBTENÇÃO DO TÍTULO \\ $\mathrm{DE}$ \\ Doutor EM CIÊNCIAS \\ Programa: Matemática \\ Orientador: Prof. Dr. Francisco César Polcino Milies
}





\section{Idempotentes Primitivos em Álgebras de Grupo Finitas e Códigos Minimais}

Esta versão da tese contém as correções e alterações sugeridas pela Comissão Julgadora durante a defesa da versão original do trabalho, realizada em 18/12/2019. Uma cópia da versão original está disponível no Instituto de Matemática e Estatística da Universidade de São Paulo.

Comissão Julgadora:

- Prof. Dr. Francisco César Polcino Milies (orientador) - IME-USP

- Prof. Dr. Raul Antonio Ferraz - IME-USP

- Prof. Dr. Edsom Ryoji Okamoto Iwaki - UFABC

- Prof. Dr. Samir Assuena - CU-FEI

- Prof. Dr. André Luiz Martins Pereira - UFRRJ 



\section{Agradecimentos}

Agradeço primeiramente a toda a minha família pelo apoio e compreensão durante todos estes anos. Agradeço aos meus pais, Nilma e Elias (em sua memória), ao meu irmão Pedro e à Maria Aparecida, que cuidou de todos nós por muitos anos. Agradeço a todos os meus antepassados, pois sem eles, este momento não existiria.

Agradeço ao meu orientador prof. Dr. Francisco César Polcino Milies pela oportunidade, pelas aulas ainda na graduação, onde aprendi sobre grupos, pelas aulas no mestrado, pela orientação no doutorado, e por superar meu medo de quedas no tatame, tendo-as repetido diversas e diversas vezes. Aos professores e colegas que exerceram papel fundamental com seus gestos, palavras, discussões e apoio. Obrigado ao prof. Raul Ferraz, por suas valiosas opiniões e por compartilhar de toda sua genialidade.

Obrigado à Edite, José, e Renato pela boa companhia durante todo o curso, sem vocês não seria o que sou. Obrigado Samir, pelas excelentes ideias e pelas discussões. Obrigado aos colegas Andrés, Tiago, Rogério e Robson. Agradecimentos especiais também à profa. Alegria Gladys Chalom pelo suporte e por toda orientação desde a graduação. Obrigado ao prof. Edson Iwaki, o qual tive o prazer de conhecer no mestrado como professor e como orientador.

Obrigado Marceli, pela compreensão, pelo suporte e pelo carinho durante esse longo e difícil período. Obrigado ao Rodrigo Ninrod por todas as conversas e por ser sempre tão suportivo. Obrigado a todos os meus amigos que compreenderam mais um período de completa ausência. Por fim, um agradecimento especial à ESEG Escola Superior de Engenharia e Gestão pela compreensão e suporte durante este período. 


\section{Resumo}

Este trabalho apresenta alguns métodos algorítmicos para determinação de famílias completas de idempotentes primitivos em componentes simples de $\mathbb{F} G$ com a hipótese de uma álgebra de grupo finita. Tais métodos viabilizam a construção de códigos minimais à esquerda em $\mathbb{F} G$. Eles são fortemente baseados em GAP e nós apresentaremos os algoritmos criados em detalhes.

Palavras-chave: Anéis de Grupo, Códigos corretores, Códigos minimais, Idempotentes Primitivos, Algoritmos, GAP. 


\section{Abstract}

In this work, we present some algoritmic methods for the determination of a complete set of primitive idempotent in simple components of a finite group algebra. Such methods provide techniques for the construction of minimal left codes in $\mathbb{F} G$. The methods are strongly based on program GAP and we present in details the routines we created.

Keywords: Group Rings, Error Correcting Codes, Minimal Codes, Primitive Idempotents, Algorithms, GAP. 


\section{Sumário}

1 Preliminares 1

1.1 Álgebra de Grupo . . . . . . . . . . . . . . . . . . . . . . . . . 1

1.2 Códigos Corretores de Erros . . . . . . . . . . . . . . . . . . 7

1.3 Pares de Shoda . . . . . . . . . . . . . . . . . . . . . . . 9 9

1.3.1 Idempotentes Primitivos em $\mathbb{F} G$ dados por Pares de Shoda . 12

1.4 Introdução ao GAP . . . . . . . . . . . . . . . . . . . . . . . 15

1.4.1 Linguagem Básica . . . . . . . . . . . . . . . . . . . . . . . 15

2 Algoritmos de busca por idempotentes não centrais primitivos 21

2.1 A detecção de um erro . . . . . . . . . . . . . . . . . . . . . 21

2.2 Algoritmos de Busca por Idempotentes . . . . . . . . . . . . . . . . 25

2.2.1 Elementos dados por endereços . . . . . . . . . . . 25

2.2.2 Analisadores de Dimensão . . . . . . . . . . . . . . . . . 29

2.2.3 Funções de Crivo . . . . . . . . . . . . . . . . . . . . . . 36

2.2.4 A Construção de um HatMap . . . . . . . . . . . . . . . . . 38

2.2.5 O Algoritmo Principal . . . . . . . . . . . . . . . 45

2.3 Uma alternativa computacional . . . . . . . . . . . . . . . 52

2.3.1 Endereços Complementares . . . . . . . . . . . . . 52 
viii SUMÁRIO

2.3.2 Método da Redução de Dimensão . . . . . . . . . . . . . . 53

2.4 Um Conjunto Completo de Idempotentes Primitivos em FG . . . . 66

3 Idempotentes em Álgebras de Grupo Não Semissimples $\quad 71$

3.0.1 A Função de Competição . . . . . . . . . . . . . . . . . . . . 71

3.0.2 Detecção de Componentes Simples . . . . . . . . . . . . 77

4 Uma Aplicação para Idempotentes de QG 87

4.1 Reconhecimento de Idempotentes Centrais Primitivos em QG . . . 87

4.2 HatMaps em QG . . . . . . . . . . . . . . . . . . . . . . 89

$\begin{array}{lll}5 & \text { Exemplos } & 91\end{array}$

6 Demais Algoritmos em Gap $\quad 99$

6.1 Lista De Funções . . . . . . . . . . . . . . . . . . . . . . . . . . . . 99

6.2 Algumas Funções Importantes . . . . . . . . . . . . . . . . . . . 103

$\begin{array}{lr}\text { Referências Bibliográficas } & 117\end{array}$ 


\section{Introdução}

Recentemente muito têm-se avançado no estudo da estrutura de anéis de grupo por meio de abordagens computacionais [16], [14]. A teoria de códigos corretores de erros tem muito em comum com a teoria de anéis de grupo. Visando atender a esta demanda, softwares tradicionais em álgebra computacional como GAP [5] e Sage [19], ganham cada vez mais suporte para o estudo específico da estrutura de anéis de grupo (Wedderga, [4]) bem como cada vez mais recursos para o estudo de códigos corretores de erros (Guava [1]).

A teoria dos códigos corretores de erros, ganhou vida no fim da década de 40 do século XX, com Richard W. Hamming. Um código é um conjunto C de palavras que são sequências $\left(a_{1}, a_{1}, . ., a_{n}\right)$ de comprimento $n$, de dígitos tomados em um conjunto finito A com q elementos chamado alfabeto. O objetivo de Hamming era criar um código que fosse capaz de detectar e corrigir um erro. Nessa época Hamming trabalhava no Bell Telephone Laboratories, ali, os computadores eram muito lentos e capazes apenas de detectar erros, mas não de corrigi-los. Quando isso acontecia o computador interrompia o programa em andamento e então muitos trabalhos eram perdidos. Em 1950 foi publicado o estudo de Hamming no Bell System Thechnical Journal, onde ele descreve um código capaz de detectar até dois erros podendo entretanto realizar a correção caso o erro fosse único. Querendo construir um código ainda mais eficiente Hamming perguntou se seria possível construir um código capaz de detectar e corrigir um erro em uma palavra com quatro dígitos de informação e três de verificação. A resposta veio no artigo A Mathematical Theory of Communication [20], de um colega de trabalho chamado Claude Shannon em 1948. Nesse artigo Shannon atribuiu o código encontrado ao próprio Hamming. O artigo de Shannon é considerado hoje como o responsável pela origem de duas teorias: 
A Teoria de Códigos Corretores de Erros e a Teoria da Informação. Pouco tempo depois, Marcel Golay, que trabalhava no Signal Corps Engineering Laboratories em Fort Monmouth, em Nova Jersey, inspirado no trabalho de Shannon publicou o artigo Notes on Digital Coding [6], de uma única página, onde descreve alguns códigos que estão entre os melhores utilizados até hoje. Desde então, a Teoria de Códigos Corretores de Erros é um amplo campo de pesquisa que vem despertando o interesse de matemáticos, engenheiros elétricos e cientistas da computação. A fim de se obter uma teoria matemática dos códigos é necessário introduzir alguma estrutura algébrica, para isso consideraremos alfabeto um corpo finito $\mathbb{F}$ com $q$ elementos e o código $\mathrm{C}$ será um subespaço vetorial do espaço $\mathbb{F}^{n}$ de dimensão $n$ sobre $\mathbb{F}$. Dessa forma $\mathrm{C}$ é dito código linear q-ário de comprimento $n$. Uma importante classe de códigos lineares são os códigos cíclicos que possuem a seguinte propriedade: Se $\left(c_{1}, \ldots, c_{n}\right)$ está em $\mathrm{C}$ então $\left(c_{n}, c_{0}, c_{1}, . ., c_{n-1}\right)$ também está em C. Os exemplos mais importantes dessa classe são os códigos de Hamming e de Golay.

É fácil verificar que o espaço vetorial $\mathbb{F}^{n}$ é isomorfo (como espaço vetorial) à álgebra de grupo $\mathbb{F} C_{n}$ onde $C_{n}$ é grupo cíclico de ordem n. Em particular os códigos cíclicos de $\mathbb{F}^{n}$ são identificados como ideais da álgebra $\mathbb{F} C_{n}$. Assim, podemos afirmar que um código cíclico pode ser realizado como um ideal de uma álgebra de grupo de um grupo cíclico. De modo geral, dizemos que um ideal de uma álgebra de grupo finita FG é um código de grupo. No caso em que a característica de F não divide a ordem de $\mathrm{G}$ a álgebra de grupo correspondente é semissimples e, portanto, o estudo dos ideais desta álgebra é equivalente ao estudo dos elementos idempotentes. O estudo de idempotentes em álgebras de grupo, determinados a partir do grupo, foi iniciada, para grupos Abelianos sobre o corpo dos números racionais em [10]. Do ponto de vista da Teoria de Códigos, o caso verdadeiramente relevante é o caso em que o corpo dos coeficientes é finito.

Trabalhos recentes vêm sido desenvolvido em torno do estudo de códigos minimais em álgebras do tipo $\mathbb{F} G$. Muito têm-se avançado no estudo de idempotentes primitivos de álgebras de grupo.

Sabin e Lomonaco em [18] forneceram um algoritmo capaz de determinar códigos minimais em $\mathbb{F} G$ quando $\mathbb{F}$ possui característica 2 e $G$ é um grupo metacíclico. Já, em [2], Bakshi e Maheshwary forneceram um algoritmo em GAP para determi- 
nação de um conjunto completo e livre de redundâncias de Pares Extremamente Fortes de Shoda.

Em [16], Olteanu apresentou uma construção alternativa ao Teorema de BrauerWitt em termos de caracteres fortemente monomiais, o que viabilizou um algoritmo para a computação das componentes de Wedderburn de álgebras de grupo semissimples finitas e em [12], Olteanu e Van Gelder apresentaram um algoritmo capaz de encontrar idempotentes primitivos em componentes simples em termos de Pares Shoda para grupos nilpotentes. Outros trabalhos destacam-se na teoria como por exemplo os de Broche e del Rio [3], [9] Jespers, e [17] Olteanu.

O trabalho, visa contribuir com o estudo em torno dos idempotentes primitivos não centrais em álgebras grupo por vias computacionais com o objetivo de preencher algumas lacunas presentes nos programas e pacotes tradicionais como GAP [5] e [4]. Nosso foco concentra-se em álgebras de grupo semissimples e finitas $\mathbb{F} G$, porém algumas aplicações são feitas para o caso não semissimples e outras podem se estender ao caso $\mathbb{Q} G$.

Os conceitos balizaram a construção dos algoritmos descritos seguem respectivamente as seguintes prioridades:

i) Descrever uma ou mais famílias completas de idempotentes primitivos da maneira mais elementar possível, sempre que possível em termos dos subgrupos de $G$.

ii) Encontrar uma família completa de idempotentes primitivos (sem necessariamente descrevê-los em termos de subgrupos de $G$ )

iii) Encontrar os idempotentes da maneira mais eficiente possível.

Ou seja, nosso produto final objetiva a descrição de famílias completas de idempotentes primitivos (não centrais) em termos dos subgrupos de $G$. Em alguns casos, algumas alternativas computacionais são fornecidas de forma que a combinação dos algoritmos apresentados resulta em uma estratégia extremamente abrangente e versátil. 
Para o caso em que $\mathbb{F} G$ não é semissimples, são apresentados algoritmos de busca por idempotentes centrais primitivos. Neste caso, uma vez obtidos os idempotentes centrais primitivos de $\mathbb{F} G$, a aplicação dos mesmos algoritmos para o caso semissimples pode nos levar à descrição de alguns ideais de $\mathbb{F} G$.

Diversos exemplos são exibidos. Neles apresentaremos uma descrição detalhada de algumas famílias de idempotentes primitivos não centrais de componentes simples de $\mathbb{F} G$.

Por fim, no apêndice, explicitamos os códigos fonte das demais funções que sejam úteis, importantes ou que foram citadas durante os capítulos anteriores. 


\section{Capítulo 1}

\section{Preliminares}

Neste capítulo, faremos uma descrição da teoria básica de Anéis de Grupo, dos conceitos elementares de Códigos Corretores no contexto de Anéis de Grupos e de Pares de Shoda.

\section{1 Álgebra de Grupo}

Nesta seção enunciaremos as principais definições e resultados básicos da teoria de Álgebras de Grupo. As definições, proposições e teoremas dessa seção, fazem referência a [13]

Definição 1.1 (Anel de Grupo) Seja $R$ anel e $G$ um grupo. Denote por $R G$ o conjunto de todas as somas finitas

$$
\sum_{g \in G} a_{g} g
$$

onde $a_{g} \in R, g \in G$. Dados $\alpha, \beta \in R G$ tais que $\alpha=\left(\sum_{g \in G} a_{g} g\right)$ e $\beta=\left(\sum_{g \in G} b_{g} g\right)$, defina a operação aditiva $\alpha+\beta$ como

$$
\left(\sum_{g \in G} a_{g} g\right)+\left(\sum_{g \in G} b_{g} g\right)=\sum_{g \in G}\left(a_{g}+b_{g}\right) g
$$

Defina também a operação multiplicativa entre os elementos $\alpha$ e $\beta$ de $R G, \alpha \beta$ como 


$$
\alpha \beta=\sum_{g, h} a_{g} b_{h} g h
$$

e a multiplicação entre um elemento $r$ de $R$ e um elemento $\alpha=\left(\sum_{g \in G} a_{g} g\right)$ de $R G$, por

$$
r \alpha=r\left(\sum_{g \in G} a_{g} g\right)=\sum_{g \in G} r a_{g} g
$$

Dizemos que o anel $R G$ diz-se anel de grupo de $G$ sobre $R$. Se $R$ for comutativo, $R G$ é uma álgebra de grupo de $G$ sobre $R$.

Definição 1.2 Um anel $R$, com unidade é dito semissimples se todo ideal à esquerda de $R$ é um somando direto. Assim se $I$ é um ideal à esquerda de $R$, então existe $J$ tal que

$$
R \cong I \oplus J
$$

onde $J$ também é um ideal à esquerda de $R$.

Teorema 1.3 (Maschke) Seja G um grupo. Então, o anel de grupo RG é semissimples se, e somente se, as seguintes condições são válidas:

i) $R$ é um anel semissimples

ii) $G$ é finito

iii) $|G|$ é invertível em $R$

Teorema 1.4 (Brauer) Seja L um ideal minimal à esquerda de um anel $R$. Então, ou $L^{2}=0$ ou $L=R e$, onde e é um idempotente de $R$

Teorema 1.5 Seja $R$ um anel com unidade. Então $R$ é semissimples se, e somente se, todos os seus ideais minimais à esquerda são gerados por um idempotente.

Definição 1.6 Seja $R$ anel com unidade, e seja $\left\{e_{1}, . ., e_{n}\right\}$ uma família de elementos de $R$ tais que:
i) $e_{i} \neq 0$ para todo $1 \leq i \leq n$
ii) $e_{i} \cdot e_{j}=\delta(i, j) e_{i}$ 
iii) $e_{1}+\ldots+e_{n}=1$

iv) Cada $e_{i}$ não pode ser escrito como soma de dois outros idempotentes não nulos ortogonais.

Então, dizemos que $\left\{e_{1}, \ldots, e_{n}\right\}$ é uma família completa de idempotentes primitivos de $R$. Ainda, um idempotente qualquer de $R$ que satisfaz iv) é chamado de primitivo.

Proposição 1.7 Seja $R$ anel semissimples com unidade. Se $R=\oplus_{i=1}^{n} L_{i}$ onde $L_{i}$ são ideais minimais à esquerda, então existe $\left\{e_{1}, \ldots, e_{t}\right\}$ uma família completa de idempotentes primitivos de $R$ tal que $t=n$. Reciprocamente se $\left\{e_{1}, \ldots, e_{n}\right\}$ é uma família completa de idempotentes primitivos de $R$ então os ideais $L_{i}=R e_{i}$,são todos minimais e $R=\oplus_{i=1}^{n} L_{i}$

Definição 1.8 (Idempotentes Centrais Primitivos) Seja $R$ anel Artiniano com unidade, e seja $\left\{e_{1}, . ., e_{n}\right\}$ uma família de elementos centrais de $R$ tais que:

i) $e_{i} \neq 0$ para todo $1 \leq i \leq n$

ii) $e_{i} \cdot e_{j}=\delta(i, j) e_{i}$

iii) $e_{1}+\ldots+e_{n}=1$

iv) Cada $e_{i}$ não pode ser escrito como soma de dois outros idempotentes centrais não nulos ortogonais.

Então, dizemos que $\left\{e_{1}, \ldots, e_{n}\right\}$ é uma família completa de idempotentes centrais primitivos de $R$. Ainda, um idempotente central qualquer de $R$ que satisfaz iv) é chamado de idempotente central primitivo.

Proposição 1.9 Seja $R$ anel Artiniano semissimples com unidade. Se $R=\oplus_{i=1}^{n} M_{i}$ onde $M_{i}$ são ideais bilaterais de $R$ então existe $\left\{e_{1}, \ldots, e_{t}\right\}$ uma família completa de idempotentes centrais primitivos de $R$ tal que $t=n$ e $M_{i}=R e_{i} 1 \leq i \leq n$. Reciprocamente se $\left\{e_{1}, \ldots, e_{n}\right\}$ é uma família completa de idempotentes centrais primitivos de $R$ então os ideais $M_{i}=R e_{i}$, são todos ideais bilaterais minimais e $R=\oplus_{i=1}^{n} M_{i}$.

Teorema 1.10 (Wedderburn-Artin) Um anel $R$ com unidade é semissimples, se e somente se, $R$ é isomorfo a uma soma direta de anéis de matrizes sobre anéis com divisão. Ou seja, se

$$
R \cong M_{n_{1}}\left(D_{1}\right) \oplus \ldots \oplus M_{n_{s}}\left(D_{s}\right)
$$


Definição 1.11 O homomorfismo $\varepsilon$ dado por

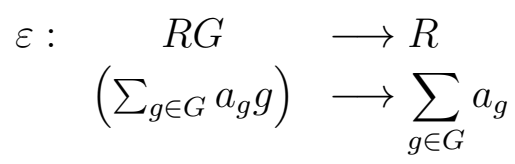

é chamado de homomor fismo de aumento de $R G$. Denote por $\Delta(G)$ o núcleo de $\varepsilon$, ou seja $\Delta(G)=\operatorname{Ker}(\varepsilon)$. O ideal $\Delta(G)$ é chamado de ideal de aumento de $R G$.

Definição 1.12 Seja $R G$ um anel de grupo e $\alpha \in R G$ tal que $\alpha=\sum_{g \in G} r_{g} g$. O conjunto $\operatorname{supp}(\alpha)=\left\{g \in G: r_{g} \neq 0\right\}$ é chamado de suporte do elemento $\alpha$.

Seja $X$ um conjunto qualquer, denotaremos por $|X|$ o número de elementos de $X$, dessa forma. $|\operatorname{supp}(g)|$ denota o número de elementos do suporte de $\alpha$

Proposição 1.13 O conjunto $\{g-1: g \in G, g \neq 1\}$ é uma $R$ - base de $\Delta(G)$.

Definição 1.14 Seja $H$ subgrupo normal de $G$. Dado o homomorfismo $\omega$, tal que

$$
\begin{aligned}
& \omega: \quad R G \quad \longrightarrow R(G / H) \\
& \left(\sum_{g \in G} a_{g} g\right) \longrightarrow \sum_{g \in G} a_{g} \bar{g}
\end{aligned}
$$

denote por $\Delta(G, H)$ o núcleo de $\omega$, ou seja $\Delta(G, H)=\operatorname{Ker}(\omega)$.

Proposição 1.15 Seja $H$ subgrupo normal de $G$ e $\mathcal{T}$ um conjunto transversal de $H$ em $G$. O conjunto $\beta_{H}=\{q(h-1): q \in \mathcal{T}, 1 \neq h \in H\}$ é uma $R-$ base de $\Delta(G, H)$.

Seja $G=C_{n}=<a \mid a^{n}=1>$ um grupo cíclico de ordem $n$ e $K$ um corpo tal que $\operatorname{car}(K) \nmid|G|$.

Considere o epimorfismo

$$
\begin{aligned}
\phi: K[X] & \longrightarrow K G \\
f & \longrightarrow f(a)
\end{aligned}
$$

Pelo Teorema do Isomorfismo, temos que 


$$
K G \cong \frac{K[X]}{K e r(\phi)}
$$

Como o $\operatorname{ker}(\phi)=<X^{n}-1>$ segue que

$$
K G \cong \frac{K[X]}{<X^{n}-1>}
$$

Se $X^{n}-1$ se decompõe em fatores irredutíveis em $K[X]$ como $X^{n}-1=f_{1} \ldots f_{t}$ então, pelo Teorema Chinês do Resto

$$
K G \cong \frac{K[X]}{<f_{1}>} \oplus \ldots \oplus \frac{K[X]}{<f_{t}>}
$$

Denotando por $\zeta_{i}$ como raiz de $f_{i}$, temos que $\frac{K[X]}{<f_{i}>} \cong K\left(\zeta_{i}\right)$ e portanto

$$
K G \cong K\left(\zeta_{1}\right) \oplus \ldots \oplus K\left(\zeta_{t}\right)
$$

De forma mais geral, podemos descrever uma álgebra de grupo Abeliana $K G$ com $G$ finito. Seja então $\Phi_{d}=\Pi_{j}\left(X-\zeta_{j}\right)$ o polinômio ciclotômico de ordem $d$ escrito em termos de todas as raízes $d$ - ésimas primitivas da unidade. Como $X^{n}-1=\Pi_{d \mid n} \Phi_{d}$, podemos reescrever a decomposição de $K G$ como

$$
K G \cong \bigoplus_{d \mid n} \bigoplus_{i=1}^{a_{d}} \frac{K[X]}{<f_{d_{i}}>} \cong \bigoplus_{d \mid n} \oplus K\left(\zeta_{d_{i}}\right)
$$

Logo, como para cada $d$, os corpos da forma $K\left(\zeta_{d_{i}}\right)$ são isomorfos.

Outra forma útil de estudar a estrutura de um anel de grupo consiste em localizar elementos, subgrupos ou componentes simples em termos de sua decomposição em componentes de Wedderburn.

Por exemplo, se $R G$ possui um elemento nilpotente, isto é, tal que $\alpha^{n}=0$ para algum $n \neq 0$, então $\alpha$ não pode estar em uma componente que seja um anel de divisão, ou seja, $\alpha \in M_{k}(D) \Rightarrow k>1$.

Temos também um resultado que nos dá informações à respeito das componentes comutativas de $R G$, para isso, vamos definir comutador e idempotente principal.

Definição 1.16 (Comutador para grupos) Seja $G$ um grupo e $x, y \in G$, denotamos por $[x, y]$ o elemento $[x, y]=x y x^{-1} y^{-1}$. Se $H$ é um subgrupo de $G$, denotamos por $[H, H]$ o conjunto $\{[x, y]: x, y \in H\}$. Denotamos por comutador de $G$ o sub- 
grupo normal $G^{\prime}=[G, G]$.

Definição 1.17 (Comutador para Anéis) Seja $R$ um anel e $x, y \in R$, denotamos por $[x, y]$ o elemento $[x, y]=x y-y x$.

Proposição 1.18 Seja $G$ grupo e $H \triangleleft G$. Então $G / H$ é Abeliano se, e somente se, $G^{\prime} \subset H$.

Definição 1.19 (Centralizador) Definimos o conjunto $\mathscr{C}_{X}(\alpha)$ como os elementos de $X$ que comutam com $\alpha$.

Definição 1.20 Seja $R G$ um anel de grupo e $X \subset R G$ um subconjunto finito de $G$. Definimos ^a função:

$$
\hat{X}= \begin{cases}\frac{1}{|X|} \sum_{x \in X} x & \text { se }|X| \text { éinvertível em } R \\ 0 & \text { caso contrário }\end{cases}
$$

Da forma como definida, se $H$ é subgrupo $\mathrm{G}$ com $R G$ semissimples, então $\hat{H}=\frac{1}{|H|} \sum_{h \in H} h$ coincide com a definição clássica. Entretanto, para o caso não semissimples, o elemento $\hat{H}$ existe, independente de $|H|$.

Definição 1.21 (Idempotente Principal) Seja $R$ um anel e $G$ um grupo finito tal que $|G|$ é invertível em $R$. O idempotente $e_{G}=\hat{G}$ é chamado idempotente principal de $R G$.

Proposição 1.22 Seja $R$ anel e $H$ subgrupo normal de um grupo $G$. Se $|H|$ é invertivel em $R$, temos que

$$
R G \cong R\left(\frac{G}{H}\right) \oplus \Delta(G, H)
$$

Corolário 1.23 Seja RG uma álgebra de grupo semissimples e $G^{\prime}$ o comutador de G. Como, por Maschke $|G|$ é invertível em $R$, e temos que

$$
R G \cong R\left(\frac{G}{G^{\prime}}\right) \oplus \Delta\left(G, G^{\prime}\right)
$$

onde $R\left(\frac{G}{G^{\prime}}\right)$ é a soma de todas as componentes simples comutativas de $\Delta\left(G, G^{\prime}\right)$ a soma de todas as outras.

Definição 1.24 Seja $G$ um grupo e $R$ um anel comutativo. Seja $\left\{C_{i}\right\}_{i \in I}$ o conjunto das classes de conjugação de $G$ que contém apenas um número finito de elementos. 
Para cada $i \in I$, defina $\gamma_{i}=\hat{C}_{i}=\sum_{x \in C_{i}} x$. Chamamos $\gamma_{i}$ de somas de classes de $G$ em $R$.

Teorema 1.25 [13] Seja $G$ um grupo e $R$ um anel comutativo. Então $\left\{\gamma_{i}\right\}_{i \in I}$ forma uma $R$-base de $\mathcal{Z}(R G)$.

Definição 1.26 Um corpo $\mathbb{K}$ é dito corpo de decomposição para um grupo finito $G$, se a álgebra $\mathbb{K} G$ é isomorfa a uma soma direta de componentes matriciais sobre $\mathbb{K}$.

\subsection{Códigos Corretores de Erros}

Definição 1.27 (Código) Seja $\mathcal{A}=\left\{a_{1}, \ldots, a_{q}\right\}$ um conjunto finito com $q$ elementos. Dizemos que $\mathcal{A}$ é dito um alfabeto cujos elementos são chamados de letras. Dizemos que um elemento $\left(c_{1}, . ., c_{n}\right) \in \mathcal{A}^{n}$ é dito uma palavra de comprimento $n$. Então, um subconjunto de $\mathcal{A}^{n}$ não trivial é dito um código de comprimento $n$ sobre o alfabeto $\mathcal{A}$.

Definição 1.28 Seja $\mathbb{F}$ um corpo finito. Um subespaço próprio de $\mathbb{F}^{n}$ é chamado de código linear sobre $\mathbb{F}$.

Definiremos agora um de nossos objetos de estudo. Os códigos em álgebras de grupo. Uma vez que uma álgebra de grupo finita $\mathbb{F} G$ é isomorfa a $\mathbb{F}^{n}$ como $\mathbb{F}$-espaços vetoriais, podemos definir um código em $\mathbb{F} G$ por meio de seus ideais.

Definição 1.29 Sejam $\mathbb{F}$ corpo e $G$ um grupo finito. Chamamos de código de grupo os ideais de $\mathbb{F} G$. Já, os ideais minimais de $\mathbb{F} G$ serão chamados de códigos minimais.

Definição 1.30 Sejam $\alpha$ e $\beta$ elementos de um código de $\mathbb{F} G$. Se $\alpha=\sum_{g \in G} \alpha_{g} g$ e $\beta=\sum_{g \in G} \beta_{g} g$, então chamamos de distância de Hamming $d(\alpha, \beta)$ o número

$$
d(\alpha, \beta)=|\operatorname{supp}(\alpha-\beta)|
$$

Definição 1.31 Dado um elemento $\alpha$ de um código de $\mathbb{F} G$, chamamos de peso de $\alpha$ o número

$$
\omega(\alpha)=d(\alpha, 0)
$$

Chamamos de peso de um código $I$ de $\mathbb{F} G$ o número

$$
\omega(I)=\min \{\omega(\alpha), \alpha \in I \backslash\{0\}\}
$$


Note que o peso de $\alpha$ é a distância de Hamming de $\alpha$ a 0 .

Definição 1.32 (Parâmetros) Dado um código $I$ de $\mathbb{F} G$, se $n=|G|, k=\operatorname{dim}_{\mathbb{F}} I$ e $d=\omega(I)$ chamamos a terna $[n, k, d]$ de parâmetros do código $I$.

Obter idempotentes em álgebras de grupo é de extrema importância para o estudo da teoria de códigos corretores de erros, uma vez que seus ideais gerados por estes idempotentes são vistos como códigos lineares sobre $\mathbb{F}$.

Um dos métodos mais tradicionais para encontrar idempotentes centrais primitivos envolve tabela de caracteres, porém métodos alternativos as evitando foram amplamente estudados recentemente. Dentre eles, o método dos Pares de Shoda se destaca por sua viabilidade computacional.

Será essencial para nosso propósito o estudo dos idempotentes centrais primitivos de $\mathbb{F} G$.

Nesse capítulo, discutiremos algumas das principais técnicas utilizadas com tal finalidade e seus desdobramentos computacionais. Notemos que uma grande ênfase será dada ao método dos Pares de Shoda devido à basicamente dois fatores: A descrição dos idempotentes primitivos pelo método dos Pares de Shoda utiliza os subgrupos de $G$ na identificação dos idempotentes centrais primitivos, além disso, nos fornece informações sobre a dimensão $n$ da componente simples e de sua respectiva dimensão sobre $\mathbb{F}$.

Como veremos mais adiante, tais informações serão fundamentais para a descrição de nosso método principal. Entretanto, mesmo que os idempotentes centrais primitivos não possam ser completamente descritos pelo método dos Pares de Shoda, faremos uso de métodos clássicos como descrição por tabela de caracteres, ou mesmo no caso não semissimples, poderemos utilizar o método da função de competição que será descrito mais adiante.

Outra vantagem sobre o método dos Pares de Shoda é que, além de descrever algumas informações sobre as componetes simples, nos permite identificar os idempotentes de forma extremamente concisa o que permite além de uma descrição limpa, identificá-los sem recorrer ao suporte do grupo, ou seja, sem escrevê-lo explicitamente. Descreveremos um conjunto completo de idempotentes primitivos (não centrais) de uma compoente de Wedderburn por uma única sequência numérica ou por um conjunto de sequências numéricas. 


\subsection{Pares de Shoda}

Seguiremos adiante com os resultados apresentadas por [12] , [17] e [9], mantendo a maioria de suas notações.

Aqui, seja $\mathbb{F}$ um corpo e $\overline{\mathbb{F}}$ seu fecho algébrico. São assumidos como caracteres em $\overline{\mathbb{F}}$, fecho algébrico de $\mathbb{F}$. Dado um grupo finito $G$ considere seu caractere $\chi$ sobre $\overline{\mathbb{F}}, e_{\mathbb{F}}(\chi)=\frac{\chi(1)}{|G|} \sum_{g \in G} \chi\left(g^{-1}\right) g$ é o idempotente primitivo central de $\overline{\mathbb{F}} G$ associado a $\chi$ e $e_{\mathbb{F}}(\chi)$ é o único idempotente primitivo central $e$ de $\mathbb{F} G$ tal que $\chi(e) \neq 0$.

O corpo dos caracteres de $\chi$ sobre $\mathbb{F}$ é definido como $\mathbb{F}(\chi)=\mathbb{F}(\chi(g): g \in G)$, isto é, uma extensão de $\mathbb{F}$ gerada pela imagem de $\chi$ sobre $\mathbb{F}$. O grupo de automorfismos $A u t(\overline{\mathbb{F}})$ age em $\overline{\mathbb{F}} G$ pela ação nos coeficientes, ou seja, $\sigma\left(\sum_{g \in G} a_{g} g\right)=$ $\Sigma_{g \in G} \sigma\left(a_{g}\right) g$, para $\sigma \in A u t(\overline{\mathbb{F}})$ e $a_{g} \in \overline{\mathbb{F}}$. Sabemos que $e_{\mathbb{F}}(\chi)=\sum_{\sigma \in G a l(\mathbb{F}(\chi) / \mathbb{F}} \sigma(e(\chi))$.

A abordagem que será apresentada consiste em uma alternativa à utilização de caracteres para determinar os idempotentes centrais primitivos de $\mathbb{F} G$ e $\mathbb{Q} G$. Como em [9] considere $G$ um grupo, com $K$ e $H$ subgrupos tais que $K \unlhd H \leq G$ . Para $\mathbb{Q} G$ o elemento $\varepsilon(H, K) \in \mathbb{Q} H \subseteq \mathbb{Q} \mathbb{G}$ é definido como:

$$
\varepsilon(H, K)= \begin{cases}\hat{K} & \text { se } H=K \\ \prod_{M / K \in \mathcal{M}(H / K)}(\hat{H}-\hat{M}) & H \neq K\end{cases}
$$

onde $\mathcal{M}(H, K)$ denota o conjunto dos subgrupos de $H / K$ normais, minimais e não triviais. Além disso, denote por $e(G, H, K)$ a soma dos diferentes $G$-conjugados de $\varepsilon(H, K)$, ou seja: se $T$ denota um transversal à direita de $\mathscr{C}(\varepsilon(H, K))$ em $G$, então

$$
e(G, H, K)=\sum_{t \in T} \varepsilon(H, K)^{t}
$$

É evidente que $e(G, H, K)$, assim como definido, é um elemento central de $\mathbb{Q} G$. Se os $G$ - conjugados de $\varepsilon(H, K)$ são ortogonais, então $e(G, H, K)$ é um idempotente central de $\mathbb{Q} G$. Tais elementos são fundamentais na construção de idempotentes centrais primitivos de $\mathbb{Q} G$.

Apesar de termos o foco voltado para idempotentes primitivos em $\mathbb{F} G$, ao final deste trabalho abordaremos alguns exemplos em $\mathbb{Q} G$, por este motivo, nos interessa ainda que de forma menos expressiva, os idempotentes centrais primitivos de $\mathbb{Q} G$. Há ainda, uma forte conexão entre os idempotentes primitivos de $\mathbb{Q} G$ e $\mathbb{F} G$ que será abordada mais para frente.

Seguindo a mesma linha de [12], vamos introduzir as notações para abordar o 
caso que mais nos interessa. Seja $\mathbb{F}=\mathbb{F}_{p^{k}}$ o corpo com $p^{k}$ elementos. Denotaremos por $p^{k}=s$. Seja $\overline{\mathbb{F}}$ o fecho algébrico de $\mathbb{F}$. Para cada inteiro $k$ coprimo com $s, \xi_{k}$ denota a raíz $k$-ésima primitiva da unidade em $\overline{\mathbb{F}}$ e $o_{k}(s)$ denota a ordem multiplicativa de $s$ módulo k. Assim: $\mathbb{F}\left(\xi_{k}\right) \cong \mathbb{F}_{s^{o}{ }_{k}(s)}$ denota o corpo de ordem $s^{o_{k}(s)}$.

Seja $\mathcal{Q}$ o subgrupo de $\mathbb{Z}_{n}^{*}$, gerado pela classe de $s$ e considere $\mathcal{Q}$ agindo em $G$ por t.g $=g^{t}$. As $s$-classe ciclotômica de $G$ são as órbitas de $G$ sobre a ação de $\mathcal{Q}$. Seja $A$ um grupo cíclico e $A^{*}$ o grupo de seus caracteres irredutíveis em $\overline{\mathbb{F}}$, seja $\mathcal{C}(A)$ o conjunto das $s$-classes ciclotômicoas de $A^{*}$, que consistem nos caracteres lineares fiéis de $A$.

Seja $K \unlhd H \leq G$ tal que $H / K$ seja cíclico de ordem $k$ e $C \in \mathcal{C}(H / K)$. Se $\chi \in C$ e $t r=t r_{\mathbb{F}\left(\xi_{k}\right) / \mathbb{F}}$ denota o traço sobre um corpo da extensão Galoisiana $\mathbb{F}\left(\xi_{k}\right) / \mathbb{F}$ então definimos:

$$
\varepsilon_{C}(H, K)=|H|^{-1} \sum_{h \in H} \operatorname{tr}(\chi(h K)) h^{-1}=[H: K] \hat{K} \sum_{X \in H / K} \operatorname{tr}(\chi(X)) h_{X}^{-1}
$$

onde $h_{X}$ denota um representante de $X \in H / K$. Observando que $\varepsilon_{C}(H, K)$ não depende da escolha de $\chi \in C$. Denote também por $e_{C}(G, H, K)$ a soma dos diferentes $G$-conjugados de $\varepsilon_{C}(H, K)$.

Um par de Shoda de $G$ é um par $(H, K)$ de subgrupos de $G$ com a propriedade que $K \unlhd H, H / K$ Abeliano, tal que um caractere linear irredutível de $H$ com núcleo $K$ induz um caractere irredutível de $G$. Como descrito em [16], há um antigo teorema de Shoda cujo resultado fornece um critério para irredutibilidade de caracteres monomiais, sabemos que $(H, K)$ é um Par de Shoda se, e somente se $K \unlhd H, H / K$ é cíclico e, se $g \in G$ e $[H, g] \cap H \subseteq K$, então $g \in H$ ou seja:

Definição 1.33 Seja $G$ um grupo finito. Um par $(H, K)$ de subgrupos de $G$ é dito Par de Shoda se satisfaz as seguintes condições:

$(S P 1) K \unlhd H$

(SP2) $H / K$ é cíclico

(SP3) Se $g \in G$ e $[H, g] \cap H \subseteq K$ então $g \in H$

Se $(H, K)$ é um par de Shoda de $G$, pode-se demonstrar que existe um único racional $\alpha$ tal que $\alpha e(G, H, K)$ é um idempotente primitivo central de $\mathbb{Q} G$ [15].

Definição 1.34 Um par de Shoda Forte de $G$ é um par $(H, K)$ de subgrupos de $G$ satisfazendo: 
(SSP1) $K \leq H \unlhd N=N_{G}(K)$

(SSP2) $H / K$ é cíclico e subgrupo Abeliano maximal de $N_{G}(K) / K$

(SSP3) Para todo $g \in G \backslash N_{G}(K), \varepsilon(H, K) \varepsilon(H, K)^{g}=0$

Proposição 1.35 [[8] 3.5.3]: As seguintes condições são equivalentes para um par $(H, K)$ de subgrupos de um grupo finito $G$ com $K \leq H$.

1. $(H, K)$ é um par de Shoda de $G, H \unlhd N_{G}(K)$ e os diferentes $G$-conjugados de $\varepsilon(H, K)$ são ortognais.

2. $(H, K)$ é um par forte de Shoda, ou seja, valem SSP1, SSP2 e SSP3.

3. Valem as propriedades:

- $\left.S S 1^{\prime}\right) H \unlhd \mathscr{C}_{G}(\varepsilon(H, K))$

- SS2') $H / K$ é cíclico e subgrupo maximal Abeliano de $\mathscr{C}_{G}(\varepsilon(H, K)) / K$

- SS3') para todo $g \in G \backslash \mathscr{C}_{G}(\varepsilon(H, K)), \varepsilon(H, K) \varepsilon(H, K)^{g}=0$

Proposição 1.36 Sejam $\left(H, K_{1}\right)$ e $\left(H, K_{2}\right)$ dois Pares de Shoda distintos de $G$, então

$$
\varepsilon\left(H, K_{1}\right) \varepsilon\left(H, K_{2}\right)=0
$$

\section{$[$ [8] 3.4.1]}

Seja ainda $K \unlhd H \leq G$ tal que $H / K$ seja cíclico (como em SP1)

Se $H$ é um subgrupo de $G, \psi$ um caractere linear de $H$ e $g \in G$, então $\psi^{g}$ denota o caractere de $H^{g}$ dado por $\psi^{g}\left(h^{g}\right)=\psi(h)$. Isso define uma ação de $G$ no conjunto dos caracteres lineares dos subgrupos de $G$. Note que se $K=\operatorname{Ker} \psi$, então $\operatorname{Ker} \psi^{g}=K^{g}$ e portanto $\psi \rightarrow \psi^{g}$ define uma bijeção entre o conjunto dos caracteres lineares de $H$ com núcleo $K$ e o conjunto dos caracteres lineares de $H^{g}$ com núcleo $K^{g}$. Essa bijeção leva classes $s$-ciclotômicas em classes $s$-ciclotômicas, induzindo a uma bijeção $\mathcal{C}(H / K) \rightarrow \mathcal{C}\left(H^{g} / K^{g}\right)$. Essa ação induz, por sua vez, a uma ação de $N=N_{G}(H) \cap N_{G}(K)$ em $\mathcal{C}(H / K)$, dessa maneira, o estabilizador de uma classe ciclotômica em $\mathcal{C}(H / K)$ independe da classe ciclotômica. Denotemos por $E_{G}(H / K)$ esse estabilizador de (quaisquer) classes ciclotômicas em $\mathcal{C}(H / K)$ sob esta ação. Ainda, segue que $\mathscr{C}_{G}\left(\varepsilon_{C}(H, K)\right)=E_{G}(H / K)$ para o caso em que $(H, K)$ é um par forte de Shoda.

Sejam $H$ e $K$ subgrupos de um grupo finito $G$ tal que $K \unlhd H$ e $H / K$ é cíclico. Assim temos que $\varepsilon(H, K) \varepsilon(H, K)^{g}=0$ para todo $g \in G$ e $g \notin \mathscr{C}_{G}(\varepsilon(H, K))$. Então $\mathscr{C}_{G}(\varepsilon(H, K))=N_{G}(K) \cdot[[8]$ Lema 3.5.2] 


\subsubsection{Idempotentes Primitivos em $\mathbb{F} G$ dados por Pares de Shoda}

Podemos enunciar o teorema principal que utilizaremos para identificar os idempotentes centrais primitivos de $\mathbb{F} G$.

Seja agora $p$ tal que $|\mathbb{F}|=p^{k}$ e seja $\mathbb{Z}_{(p)}$ a localização de $\mathbb{Z}$ em $p$. Então podemos enxergar $\mathbb{F}_{p}$ como elementos de $\mathbb{Z}_{(p)}$ denotando por $\bar{x}$ a imagem de $x \in \mathbb{Z}_{(p)}$ em $\mathbb{F}_{p}$. Podemos também extender esta notação à projeção de $\mathbb{Z}_{(p)}$ em $\mathbb{F}_{p} G$. Note que tanto $\varepsilon(G, N)$ pertence à $\mathbb{Z}_{(p)}$ (pois $N \unlhd G$ ) como $e(G, K, H)$ (para $K \unlhd H \leq G$ ). Então $\overline{\varepsilon(H, K)}$ é um idempotente de $\mathbb{F}_{p} G$ e se $(H, K)$ é um par forte de Shoda então $\overline{e(G, H, K)}$ é um idempotente central de $\mathbb{F}_{p} G$.

Proposição 1.37 Seja $G$ finito, Abeliano de ordem $n$ e $\mathbb{F}$, um corpo finito de ordem $q$ tal que $\operatorname{mdc}(q, n)=1$ então $(N, C) \rightarrow \varepsilon_{C}(G, N)$ é uma bijeção do conjunto de pares $(N, C)$ com $N \unlhd G$ tais que $G / N$ é cíclico e $C \in C_{q}(G / N)$ no conjunto dos idempotenets centrais primitivos de $\mathbb{F} G$. Além disso, para cada $N \unlhd G e$ $C \in \mathcal{C}(G / N), \mathbb{F} G \varepsilon_{C}(G, N) \cong \mathbb{F}\left(\xi_{k}\right)$, onde $k=[G: N]$.

Como consequência, um importante resultado afirmando que o idempotente independe da escolha de $C$.

Corolário 1.38 Se $N \unlhd G$ é tal que $G / N$ é cíclico e $C \in \mathcal{C}(G / N)$ então $\varepsilon_{C}(G, N)$ é um idempotente primitivo central de $\mathbb{F} G$ e $\mathbb{F} G \varepsilon_{C}(G, N) \cong \mathbb{F}\left(\xi_{k}\right)$, onde $k=[G$ : $N]$, além disso, se $D$ é um outro elemnento de $\mathcal{C}(G / N)$ então $\varepsilon_{C}(G, N)=\varepsilon_{D}(G, N)$ se e somente se $C=D$.

A próxima proposição é fundamental para a demonstração de diversos resultados.

Proposição 1.39 [ [3] - Lema 6]

1 - Seja $N \unlhd G$ tal que $G / N$ é cíclico, então

$$
\overline{\varepsilon(G, N)}=\sum_{C \in \mathcal{C}(G / N)} \varepsilon_{C}(G, N)
$$

2- Seja $K \unlhd H \leq G$ tal que $H / K$ é cíclico e $R$ um conjunto de representantes da ação de $N_{G}(K)$ em $\mathcal{C}(H / K)$, então

$$
\overline{e(G, H, K)}=\sum_{C \in R} \varepsilon_{C}(G, H, K)
$$


Teorema 1.40 [Teorema [12]] Seja $G$ um grupo finito e $\mathbb{F}$ um corpo finito de ordem s tal que $\mathbb{F} G$ seja semissimples. Seja $(H, K)$ um par forte de Shoda de $G$ e $C \in \mathcal{C}(H / K)$. Então e $e_{C}(G, H, K)$ é um idempotente primitivo central de $\mathbb{F} G$ e

$$
\mathbb{F} G e_{C}(G, H, K) \cong M_{[G: H]}\left(\mathbb{F}_{s^{o /[E: H]}}\right)
$$

onde $E=E_{G}(H / K)$ e o denota a ordem multiplicativa de s módulo $[H: K]$.

Definição 1.41 [Grupo Fortemente Monomial] Seja $\chi$ um caractere irredutível (complexo) de $G$. Então $\chi$ é dito fortemente monomial se há um par forte de Shoda $(H, K)$ de $G$ e um caractere linear $\theta$ de $H$ com núcleo $K$ tal que $\chi=\theta^{G}$, o caractere induzido de $G$. O grupo $G$ então é dito fortemente monomial se todo caractere irredutível de $G$ é fortemente monomial.

Corolário 1.42 Se $G$ é um grupo fortemente monomial e $\mathbb{F}$ é um corpo finito de ordem s tal que $\mathbb{F} G$ é semissimples, então todo idempotente primitivo central de $\mathbb{F} G$ é da forma e $e_{C}(G, H, K)$ para $(H, K)$, par forte de Shoda de $G$ e $C \in \mathcal{C}(H / K)$. Além, disso, para cada par forte de Shoda $(H, K)$ de $G$ e todo $C \in \mathcal{C}(H / K)$

$$
\mathbb{F} G e_{C}(G, H, K) \cong M_{[G: H]}\left(\mathbb{F}_{s^{o /[E: H]}}\right)
$$

onde $E=E_{G}(H / K)$ e o a ordem multiplicativa de s módulo $[H: K]$.

O resultado anterior relaciona os Pares de Shoda fortes com os idempotentes centrais primitivos de $\mathbb{F} G$ e por sua vez, com as componentes simples deste anel. O próximo resultado, estabelece entretanto, uma relação entre os Pares de Shoda fortes e idempotentes primitivos não centrais da uma componentes simples de $\mathbb{F} G$ como descrita no teorema anterior.

Teorema 1.43 [12]. Seja $\mathbb{F}$ um corpo finito e $G$ um grupo finito nilpotente tal que $\mathbb{F} G$ seja semissimples. Seja $(H, K)$ um par forte de Shoda de $G, C \in \mathcal{C}(H / K)$ e seja $e_{C}=e_{C}(G, H, K), \varepsilon_{C}=\varepsilon_{C}(H, K), H / K=<\bar{a}>, E=E_{G}(H / K)$. Seja $E_{2} / K$ e $H_{2} / K=<\overline{a_{2}}>$ (respectivamente $E_{2^{\prime}} / K$ e $H_{2^{\prime}} / K=<\overline{a_{2^{\prime}}}>$ ) as 2-partes (respectivamente $2^{\prime}$-partes) de $E / K$ e $H / K$ respectivamente. Então $<\overline{a_{2^{\prime}}}>$ tem um complemento cíclico $<\overline{b_{2^{\prime}}}>$ em $E_{2^{\prime}} / K$.

Um conjunto completo de idempotentes primitivos de $\mathbb{F} G e_{C}$ consiste nos conjugados de

$$
\beta_{e_{C}}=\hat{b_{2^{\prime}}} \beta_{2} \varepsilon_{C}
$$

pelos elementos de $T_{e_{C}}=T_{2^{\prime}} T_{2} T_{E}$, onde $T_{2^{\prime}}=\left\{1, a_{2^{\prime}}, a_{2^{\prime}}^{2} \ldots, a_{2^{\prime}}^{\left[E_{2^{\prime}}: H_{2^{\prime}}\right]-1}\right\}, T_{E}$ denota 
um transversal à direita de $E$ em $G$ e $\beta_{2}$ e $T_{2}$ são dados conforme os seguintes casos:

1. Se $H_{2} / K$ possui um complemento $M_{2} / K$ em $E_{2} / K$ então $\beta_{2}=\hat{M}_{2}$. Além disso, se $M_{2} / K$ é cíclico, então existe $b_{2} \in E_{2}$ tal que $E_{2} / K$ é dado pela seguinte apresentação:

$$
<\overline{a_{2}}, \overline{b_{2}} \mid{\overline{a_{2}}}^{2^{n}}={\overline{b_{2}}}^{2^{k}}=1, \overline{a_{2}} \bar{b}^{-}={\overline{a_{2}}}^{r}>
$$

e se $M_{2} / K$ não é cíclico, então existem $b_{2}, c_{2} \in E_{2}$ tais que $E_{2} / K$ é dado pela seguinte apresentação.

$$
<\overline{a_{2}}, \overline{b_{2}}, \overline{c_{2}} \mid{\overline{a_{2}}}^{2^{n}}={\overline{b_{2}}}^{2^{k}}={\overline{c_{2}}}^{2}=1,{\overline{a_{2}}}^{\overline{b_{2}}}={\overline{a_{2}}}^{r},{\overline{a_{2}}}^{\overline{c_{2}}}={\overline{a_{2}}}^{-1},\left[\overline{b_{2}}, \overline{c_{2}}\right]=1>,
$$

com $r \equiv 1(\bmod 4)$, ou equivalentemente ${\overline{a_{2}}}^{2^{n-2}}$ é central em $E_{2} / K$. Então:

a) $T_{2}=\left\{1, a_{2}, a_{2}^{2}, \ldots, a_{2}^{2^{k}-1}\right\}$, se ${\overline{a_{2}}}^{2^{n-2}}$ é central em $E_{2} / K$ (a menos que $\left.n \leq 1\right)$ e $M_{2} / K$ é cíclico, $e$

b) $T_{2}=\left\{1, a_{2}, a_{2}^{2}, \ldots, a_{2}^{d / 2-1}, a_{2}^{2^{n-2}}, a_{2}^{2^{n-2}}, a_{2}^{2^{n-2}+1}, . ., a_{2}^{2^{n-2}+d / 2+1}\right\}$, onde $d=\left[E_{2}\right.$ : $\left.\mathrm{H}_{2}\right]$, caso contrário.

2. $\mathrm{Se} \mathrm{H}_{2} / K$ não possui complemento em $E_{2} / K$, então, existem $b_{2}, c_{2} \in E_{2}$ tais que $E_{2} / K$ é dado por

$$
\begin{aligned}
& <\overline{a_{2}}, \overline{b_{2}}, \overline{c_{2}} \mid{\overline{a_{2}}}^{2^{n}}={\overline{b_{2}}}^{2^{k}}=1,{\overline{c_{2}}}^{2}={\overline{a_{2}}}^{2^{n-1}},{\overline{a_{2}}}^{\overline{b_{2}}}={\overline{a_{2}}}^{r}{\overline{a_{2}}}^{\bar{c}}=\overline{a_{2}}{ }^{-1},\left[\overline{b_{2}}, \overline{c_{2}}\right]=1>, \\
& \text { com } r \equiv 1 \text { (mod 4). Nesse caso } \beta_{2}={\hat{b_{2}}}_{\frac{1+x a_{2}^{2 n-2}+y a_{2}^{2 n-2} c_{2}}{2}} e
\end{aligned}
$$

$$
T_{2}=\left\{1, a_{2}, a_{2}^{2}, \ldots, a_{2}^{2^{k}-1}, c_{2}, c_{2} a_{2}, c_{2} a_{2}^{2}, \ldots, c_{2} a_{2}^{2^{k}-1}\right\},
$$

com $x, y \in \mathbb{F}$, satisfazendo $x^{2}+y^{2}=-1$ e $y \neq 0$.

Ressalvas são feitas por [18, remark 3.4] quanto ao processo de obtenção de soluções para a equação $x^{2}+y^{2}=-1$ em $\mathbb{F}$. 


\subsection{Introdução ao GAP}

Recentemente, muito vem se falando sobre matemática computacional. Tendo nascida para resolver problemas envolvendo cálculos matemáticos, a relação entre computação e matemática remete à época de Turing e, desde então, tal relação nunca deixou de existir. Hoje, quase cem anos depois, a computação transcende a simples aritmética e pode ser utilizada como ferramente no estudo das mais diversas áreas do conhecimento. Hoje, uma das ferramentas mais importantes para teóricos em álgebras de grupo que desejam utilizar-se de computadores para debruçarse sobre tais objetos, é o sistema GAP - Groups, Algorithms, Programming - a System for Computational Discrete Algebra. [5].

O sistema GAP fornece uma linguagem de programação voltada para o estudo de álgebras discretas, com ênfase em Teoria de Grupo Computacional. Porém, diversos pacotes são oferecidos aos usuários de GAP possibilitando sua utilização em diversas outras áreas da Álgebra, como por exemplo, o pacote Wedderga [4] Wedderburn Decomposition of Group Algebras, o pacote LAGUNA - Lie AlGebras and UNits of group Algebras, e o pacote GUAVA-A GAP package for computing with error-correcting codes [1].

Nos capítulos que se seguem, construiremos todos os nossos algoritmos em linguagem GAP, utilizando os pacotes Wedderga, Guava e Laguna. Por isso, foneceremos uma breve introdução à linguagem e listaremos algumas das funções já existentes no GAP e nos pacotes que serão utilizadas.

\subsubsection{Linguagem Básica}

Sobre a execução de comandos É importante ressaltar que todos os comandos devem terminar com "; " ou ";;". No caso de ; ( ponto-e-vírgula) o resultado da executação é exibido, já no caso de ;; - (duplo ponto-e-vírvula) o resultado da execução é omitido. Comentários devem ser feitos após "\#” e não serão lidos como comandos.

Devemos primeiramente carregar os pacotes Wedderga, Guava e Laguna utilizados por meio de LoadPackage, em nosso caso, basta executar o comando LoadPackage("Wedderga") que os três pacotes serão todos carregados, como em

1 gap > LoadPackage("Wedderga");

Objetos e atribuição de variáveis A qualquer momento um objeto pode ser exibido ou uma variável declarada. Para declarar uma variável, utiliza-se ":=" 
(dois-pontos igual). Se uma variável possui já possui valor declarado, uma nova declaração elimina seu valor antigo.

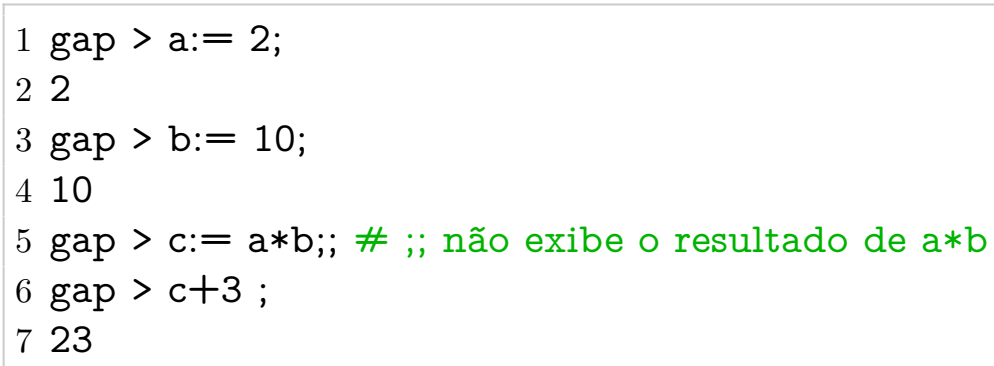

Listas Listas podem ser obtidas por meio de List(Lista, função opcional). Uma lista é um objeto da forma $A=\left[a_{1}, \ldots, a_{i}, \ldots, a_{j}, \ldots, a_{n}\right]$, onde $a_{i}$ é um objeto qualquer, não necessariamente da mesma natureza de $a_{j}$, isto é , $a_{i}$ pode ser uma função enquanto $a_{j}$, pode, por exemplo, ser um outro conjunto.

Se $A$ é uma lista, seu i-ésimo elemento pode ser acessado por meio de $A[i]$. Por exemplo, se $A=[10,9,8,7]$ então seu segundo elemento pode ser obtido por meio de $A[2]$. Opcionalmente, se uma função f for fornecida no segundo argumento, então o resultado retornará a imagem de $\mathrm{f}$ por todos os valores de $A$. Funções simples podem ser escritas como $x->$ Expressão como exemplo, a função $f(x)=x^{2}$ pode ser representada como " $x->x^{\wedge} 2$ ".

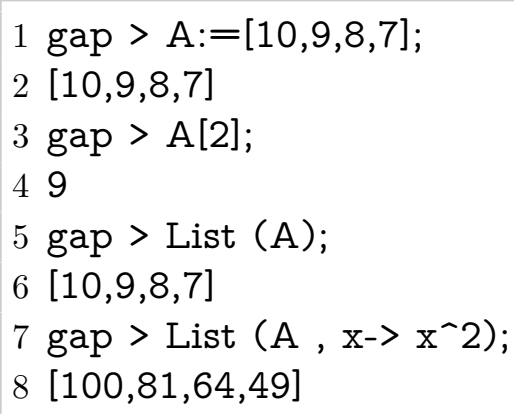

É importante ressaltar que comutadores, índices de subgrupos e listas possuem a mesma notação dada por colchetes, entretanto serão utilizadas em contextos distintos não levando a dupla interpretação.

Loops e Recorrências. Funções do tipo while, for possuem estruturas semelhantes. Devem sempre ser iniciadas seguindo-se de uma condição e o termo do, e encerradas com od; 


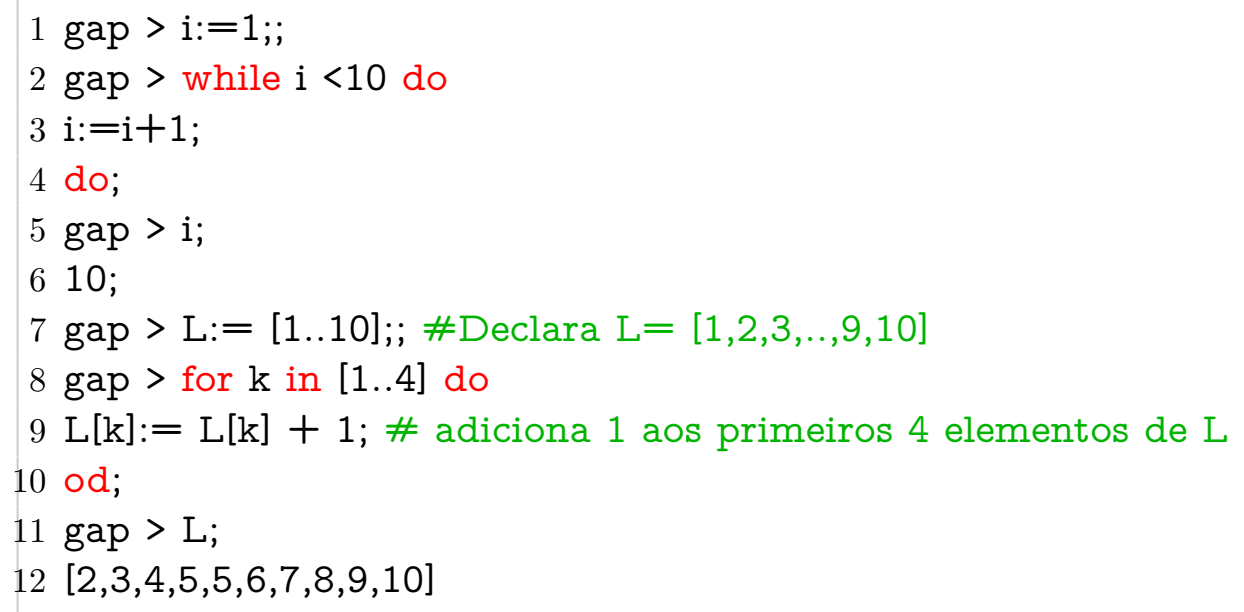

Condicional if/else. As condicionais if/else possuem estrutura semelhante às funções while e for. Devem ser seguidas de uma expressão avaliada em true ou em false, seguidas pelo termo then e encerradas por $f i$;

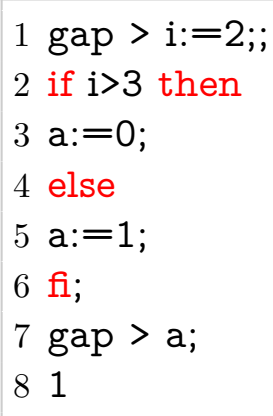

Criando novas funções e rotinas. uma função pode ser declarada e atribuída a uma variável por function(atributos) e encerrada por end;sendo que uma função pode possuir variáveis locais declaradas como local Variável1, Variável2, .., Variáveln; e function retorna ao sistema o objeto emitido por return;

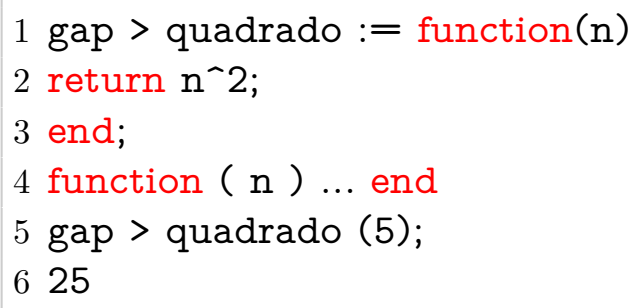


Lidando com Anéis de Grupo. Após carregados os pacotes necessários, pode declarar um anel de grupo $R G$ por meio de GroupRing(Anel, Grupo). Vamos utilizar frequentemente os anéis dados por $G F(q)$ (Galois Field - $\mathbb{F}_{q}$ ) e por Rationals (o corpo $\mathbb{Q}$ ). Já os grupos podem ser obtidos por meio de um quociente de grupos livres, por meio de seus geradores ou diversas outras formas permitidas pelo GAP. Sempre que possível, vamos utilizar a biblioteca AllSmallGroups(Ordem)[Núm]. O comando AllSmallGroups retorna uma lista com todos os grupos dada uma determinada ordem presentes na biblioteca do GAP . Dado um determinado grupo $G$, pode-se tentar identificá-lo via IdSmallGroup, retornando assim sua classificação na biblioteca Ainda, para grupos de ordem pequena, pode-se tentar identificar sua estrutura via StructureDescription.

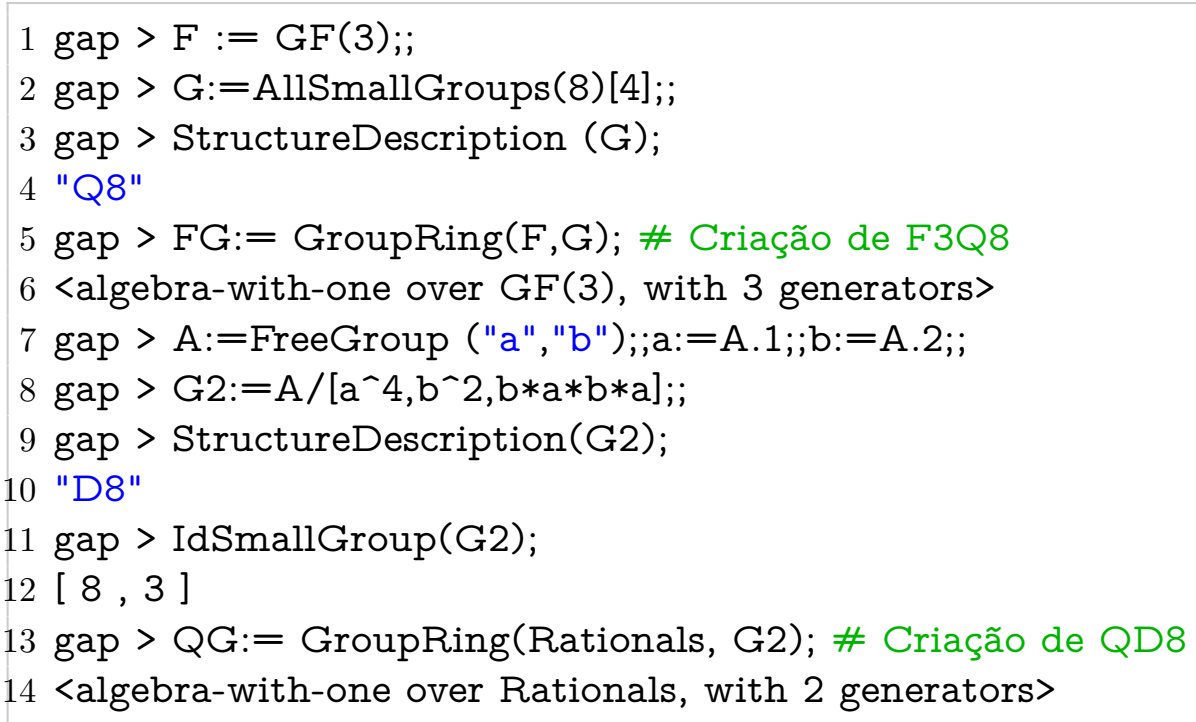

Dado uma álgebra de grupo semissimples, podemos obter seus idempotentes centrais primitivos por meio de PrimitiveCentralIdempotentsByCharacterTable, bem como obter via $W$ edderburnDecomposition $(F G)$ informações sobre suas componentes de Wedderburn, estudando assim sua estrutura.

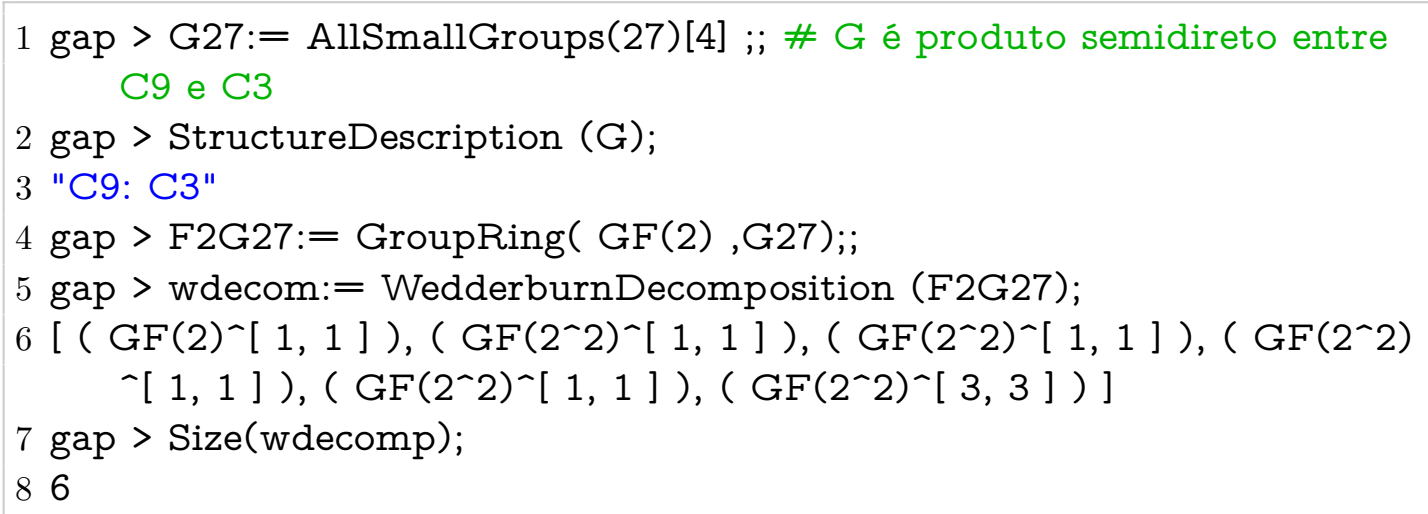


9 gap > wdecomp[6];

$10\left(\mathrm{GF}\left(2^{\wedge} 2\right)^{\wedge}[3,3]\right)$

Demais comandos estão listados no último capítulo e serão utilizados ao longo deste trabalho. Como já mencionado, uma ressalva importante quanto à notação, o qual utilizaremos o formato função para funções e comandos nativos do GAP e a notação função para funções e algoritmos construídos por nós, ou seja, não oferecidas de forma nativa pelo GAP ou presentes em algum de seus pacotes.

No decorrer dos próximos capítulos faremos uso do termo Lista nos referindo como uma $n$-úpla de objetos indexados. Dado um grupo $G$, vamos nos referir como $S u b G$ à lista formada por seus subgrupos. 


\section{Capítulo 2}

\section{Algoritmos de busca por idempotentes não centrais primitivos}

\subsection{A detecção de um erro}

A descrição das álgebras de grupo semissimples sobre corpos finitos $\mathbb{F}$ e extensões finitas de $\mathbb{Q}$ por vias computacionais foi um problema resolvido completamente em [16]. Já, para calcular idempotentes centrais primitivos, o algoritmo apresentado envolve o método da tabela de caracteres. Posteriormente, apresentou-se um segundo método, baseado nos Pares de Shoda como alternativa para o caso em que $G$ é fortemente monomial, como discutido no capítulo anterior.

O programa GAP [5] oferece no pacote Wedderga [4] uma rotina chamada de PrimitiveIdempotentsNilpotent que é consequência da determinação de idempotentes não centrais primitivos obtida por Olteanu e Van Gelder em [17] para álgebras de grupos finitas em grupos nilpotentes. No entanto, o código fonte correspondente apressenta um erro. Olteanu e Van Gelder utilizaram esta rotina em [12] para determinar códigos em $\mathbb{F}_{2} G$, onde $G=G_{[27,4]}$, ou seja

$$
G=<a, b \mid a^{9}=1, b^{3}=1, b a=a^{4} b>
$$

Tem-se que

$$
\mathbb{F}_{2} G \cong \mathbb{F}_{2} G \cong \mathbb{F}_{2} \oplus 4 \mathbb{F}_{4} \oplus M_{3}\left(\mathbb{F}_{4}\right)
$$

O idempotente que gera a componente isomorfa a $M_{3}\left(\mathbb{F}_{4}\right)$ é obtido à partir do 
par de Shoda Forte $(H, K)=(<a>, 1)$.

Nessa componente deveriam existir idempotentes primitivos não centrais que geram ideais à esquerda de dimensão 3 sobre $\mathbb{F}_{4}$, ou seja, 6 sobre $\mathbb{F}_{2}$. Porém, em [12], aplicando a rotina PrimitiveIdempotents Nilpotent, se executou as seguintes instruções:

1 gap > A:= FreeGroup ("a", "b");;a:=A.1;;b:=A.2;;

2 gap $>\mathrm{G}:=\mathrm{A} /\left[\mathrm{a}^{\wedge} 9, \mathrm{~b}^{\wedge} 3, \mathrm{~b} * \mathrm{a} * \mathrm{~b}^{\wedge}(-1) * \mathrm{a}^{\wedge}(-4)\right] ;$

3 gap $>F:=G F(2) ;$;

4 gap $>F G:=\operatorname{GroupRing}(F, G) ;$;

5 gap $>\mathrm{S}:=\operatorname{AsSet}(G) ;$

6 gap $>\mathrm{H}:=\operatorname{Subgroup}(\mathrm{G},[\mathrm{G} .1]) ;$;

7 gap $>K:=\operatorname{Subgroup}(G,[]) ; ;$

8 gap $>\mathrm{N}:=$ Normalizer $(G, K) ;$;

9 gap > epi:= NaturalHomomorphismByNormalSubgroup $(\mathrm{N}, \mathrm{K})$;;

10 gap > QHK:= Image (epi, $\mathrm{H}) ;$;

11 gap > gq:= MinimalGeneratingSet(QHK )[1];;

12 gap > C:= CyclotomicClasses(Size(F), Index $(H, K))[2] ; ;$

13 gap > P:= PrimitiveIdempotentsNilpotent (FG, H,K,C,[epi ,gq]);

14 gap $>$ e:=P[1];

Com o primeiro idempotente, $e=p[1]$ executa-se

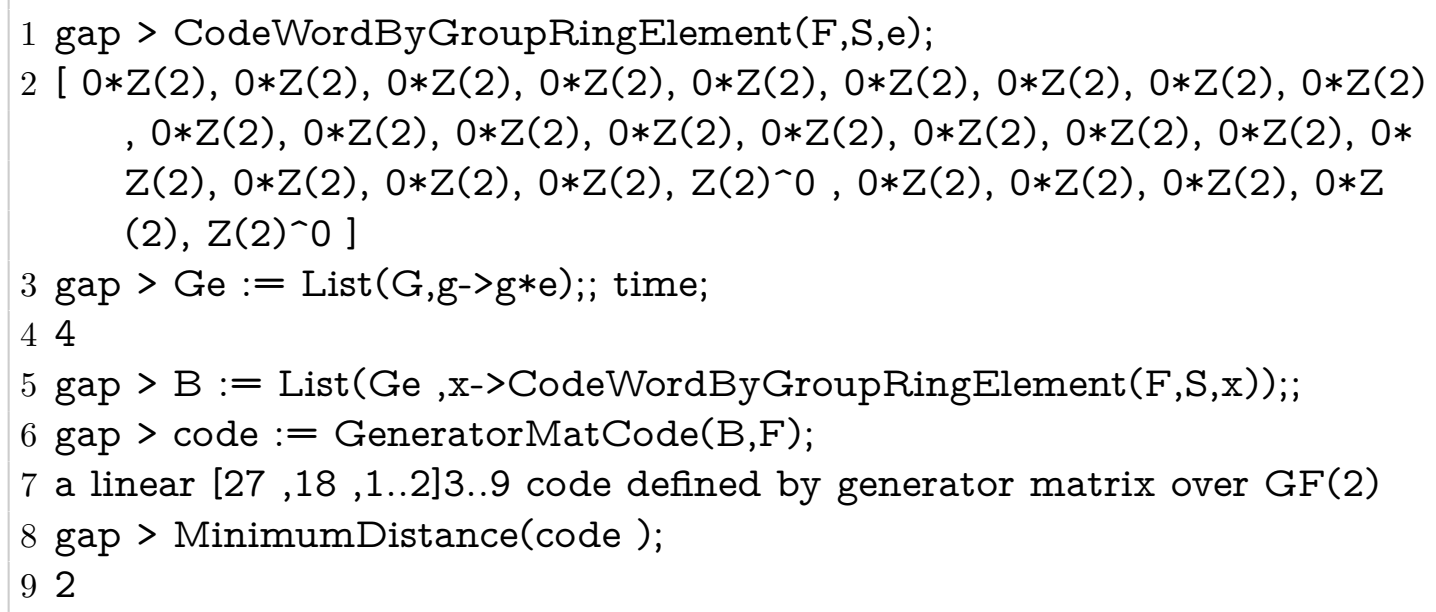

e se obtém que o código $\mathbb{F} G e$ tem parâmetro $[27,18,2]$, no entanto, 18 é a dimensão de toda a componente simples correspondente e ideais minimais nela deveriam ter dimensão 6 .

$\mathrm{Na}$ verdade é fácil verificar diretamente que $e$ não é nem mesmo um elemento idempotente, por exemplo, executando IsIdempotent $(e)$. 


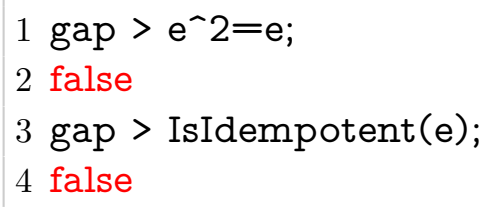

De fato, os três elementos dados como idempotentes pela rotina são:

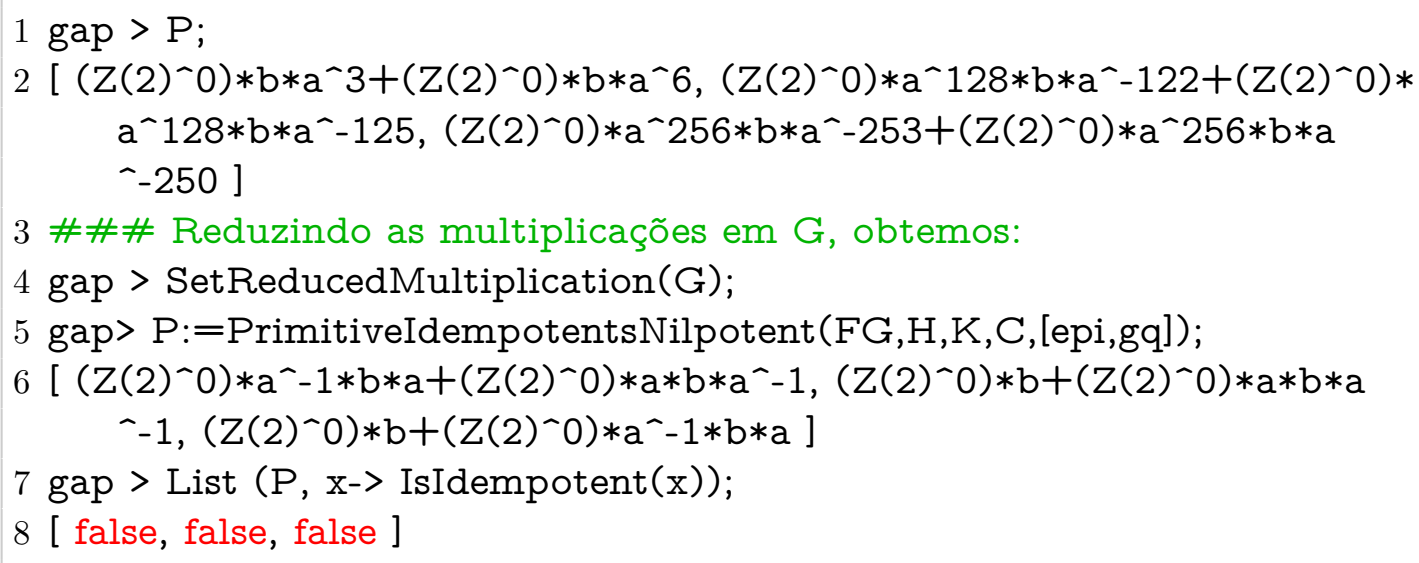

Assim, os elementos $e_{1}=a^{-1} b a+a b a^{-1}, e_{2}=b+a b a^{-1}$ e $e_{3}=b+a^{-1} b a$, dados pelo cálculo acima não são idempotentes.

Uma forma de reparar o erro consiste em trocar o trecho final do código fonte:

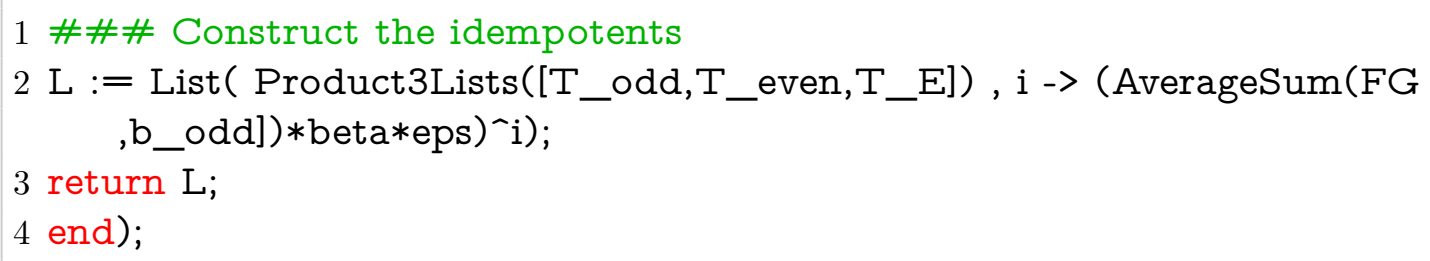

Pelo seguinte código fonte:

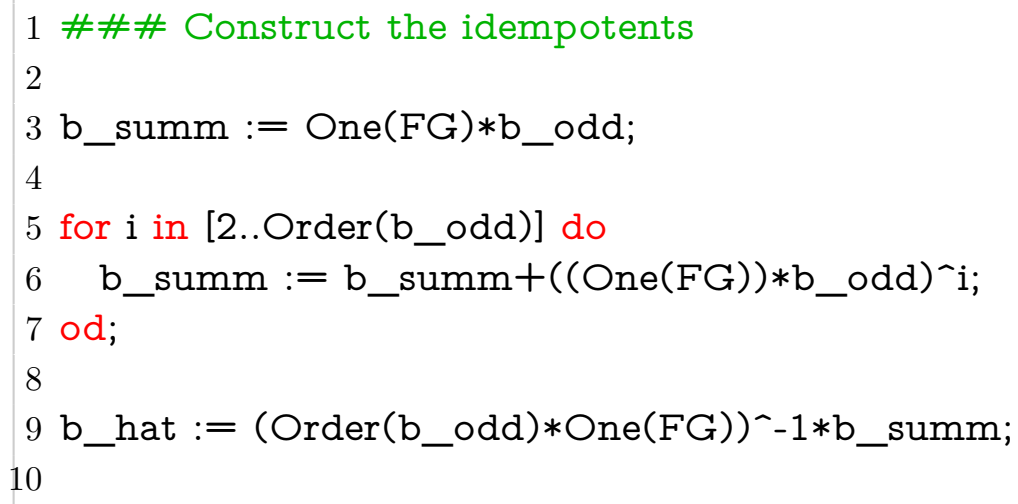


$11 \mathrm{~L}:=$ List( Product3Lists $\left(\left[\mathrm{T} \_\right.\right.$odd, $\left.\left.\mathrm{T}_{\text {_even, }} \mathrm{T}_{-} \mathrm{E}\right]\right)$ ), i $\rightarrow$ (b_hat*beta*eps )$\left.^{\sim} \mathrm{i}\right)$;

12

13 return L;

14 end);

A seguinte alteração produz os elementos:

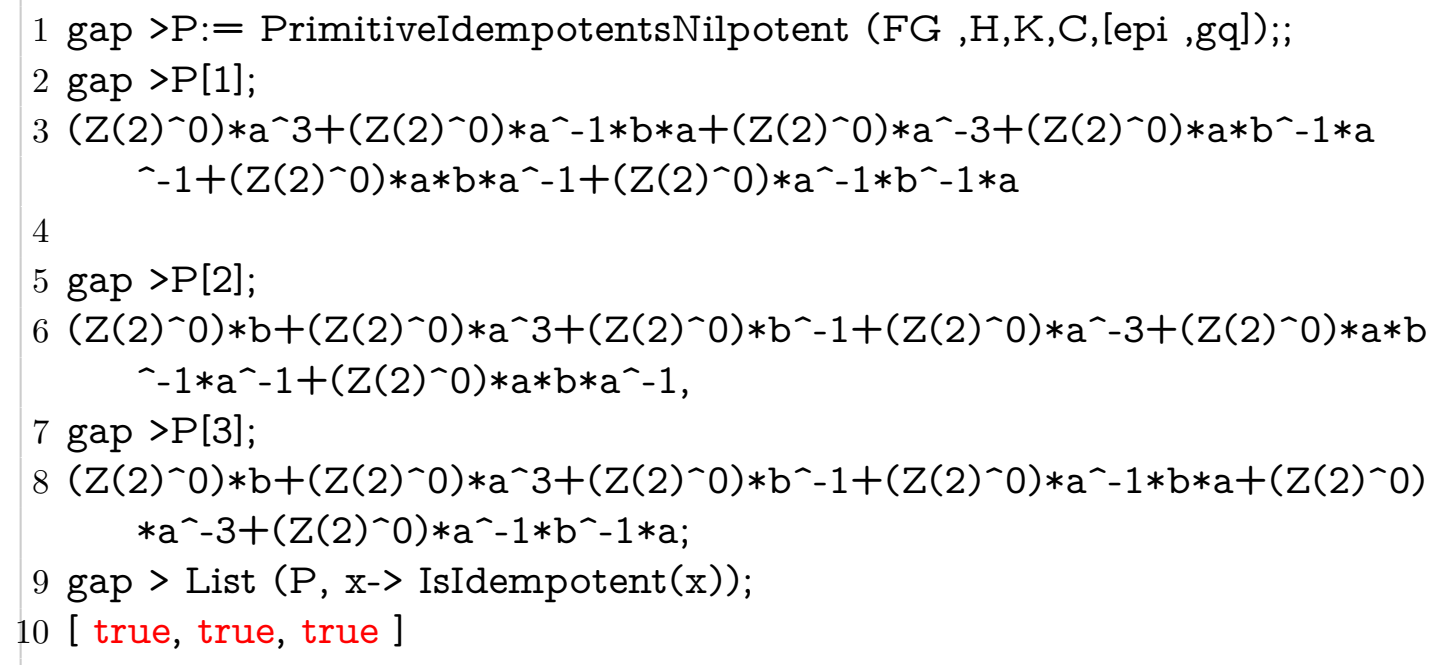

Reavaliando o código fonte obtido:

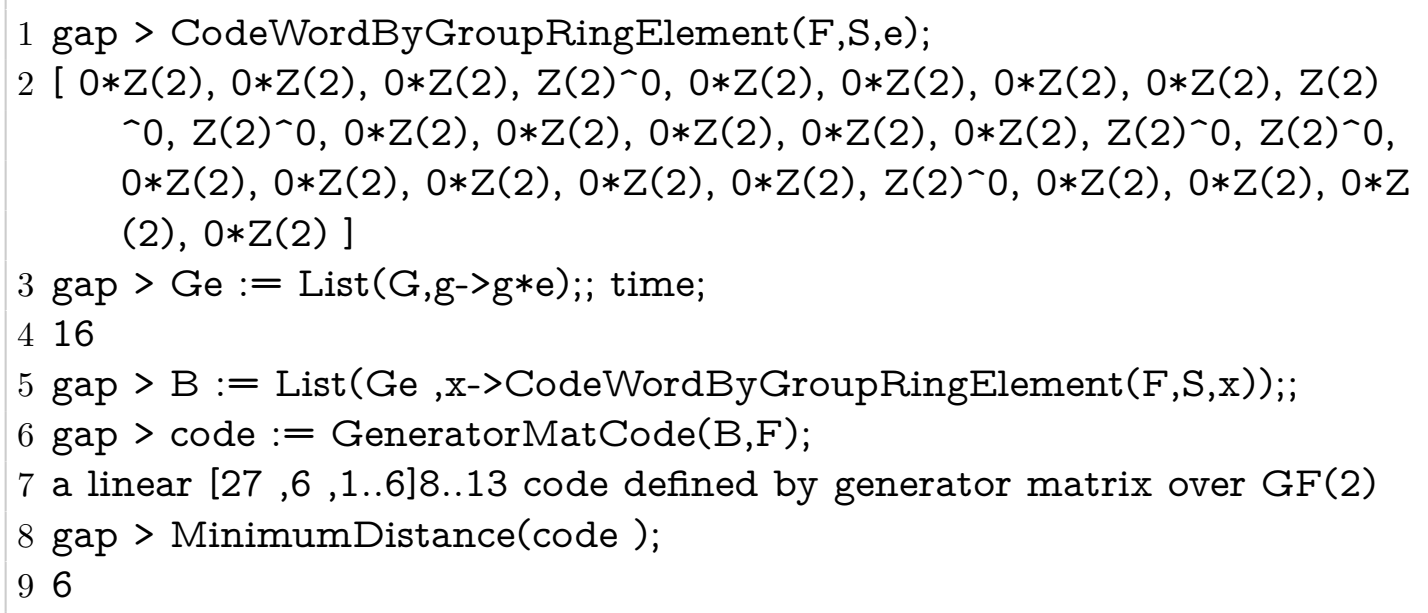

Portanto, resulta um código $[27,6,6]$ sobre $\mathbb{F}_{2}$, como era de se esperar. 


\subsection{Algoritmos de Busca por Idempotentes}

\subsubsection{Elementos dados por endereços}

O estudo do problema descrito anterioremente motivou a criação das técnicas que apresentaremos. Ao nos depararmos com os algoritmos disponíveis, dois problemas imediatamente se ressaltaram: o problema da descrição do idempotente e a limitação da técnica que se aplica a apenas uma classe específica de grupo.

O primeiro problema refere-se à dificuldade de leitura que pode surgir da própria natureza dos elementos em questão, ou seja, ao trabalharmos com uma álgebra de grupo, seus elementos podem possuir um suporte do tamanho do próprio grupo. Uma frequente inconveniência dos algoritmos tradicionais que determinam elementos de uma álgebra de grupos $R G$ é a descrição explícita dos elementos de $R G$. Mesmo que descrever estes elementos de forma explicita seja uma consequência imediata do propósito dos algoritmos. Descrever tais elementos em termos de seu suporte em $G$ pode ser de difícil leitura humana, principalmente para grupos de ordem grande. Tais descrições podem ainda ser piores em álgebras sobre um corpo de ordem $p^{k}$.

Dessa forma, os resultados obtidos podem ser de difícil interpretação, bem como difíceis de serem manipulados e utilizados em futuros estudos.

O segundo problema refere-se às limitações impostas à classe do grupo. No caso, o algoritmo PrimitiveIdempotentsNilpotent depende de G ser um grupo nilpotente bem como de um idempotente central primitivo descrito por um Par de Shoda Forte.

Com as construções que descreveremos à seguir, será possível a criação de um novo e versátil algoritmo capaz de descrever os idempotentes primitivos (não centrais) de uma componente simples $\mathbb{F} G e$ sem necessariamente uma restrição quanto ao tipo de grupo, possibilitando assim, um estudo diversificado de tipos de álgebras de grupo. Nosso foco principal serão as álgebras de grupo $\mathbb{F} G$ finitas, entretanto, em muitos casos, obteremos sucesso também em $\mathbb{Q} G$.

Considere agora a seguinte situação: Suponha haver $k$ idempotentes $e_{1}, \ldots, e_{k}$ que comutam dois a dois. 
Temos que

$$
1=e_{i}+\left(1-e_{i}\right), 1 \leq i \leq k
$$

Multiplicando ordenadamente estas equações, temos

$$
\begin{aligned}
1= & e_{1} . . e_{k}+e_{1} \ldots\left(1-e_{k}\right)+\ldots+e_{1} . .\left(1-e_{k-1}\right) e_{k}+ \\
+ & e_{1} \ldots\left(1-e_{k-1}\right)\left(1-e_{k}\right)+\ldots+\left(1-e_{1}\right) \ldots\left(1-e_{k}\right)
\end{aligned}
$$

Ou seja, escrevemos a identidade da álgebra de grupo como soma de idempotentes dois a dois ortogonais.

De fato, sejam $e_{i}^{\prime}$ e $e_{j}^{\prime}$ dois termos quaisquer da soma acima, e denote por $e^{+} \mathrm{e}$ por $e^{-}$elementos do tipo $e^{+}=e$ e $e^{-}=1-e$ temos que ambos são idempotentes da forma

$$
e_{1}^{ \pm} \ldots e_{k}^{ \pm}
$$

Se $e_{i}^{\prime} \neq e_{j}^{\prime}$ então existe pelo menos um $e_{t}$ tal que se $e_{t}^{+}$está em $e_{i}^{\prime}$ então $e_{t}^{-}$está em $e_{j}^{\prime}$ e, se $e_{t}^{-}$está em $e_{i}^{\prime}$ então $e_{t}^{+}$está em $e_{j}^{\prime}$. Dessa forma, segue que $e_{i}^{\prime} \cdot e_{j}^{\prime}=\delta(i, j) e_{i}^{\prime}$

Se e então denota um idempotente central de uma componente simples $\mathbb{F} G \mathbf{e} \cong$ $M_{n}\left(\mathbb{F}_{q}\right)$,temos que

$$
\begin{aligned}
\mathbf{e}=1 \mathbf{e}= & e_{1} \ldots e_{k} \mathbf{e}+e_{1} \ldots\left(1-e_{k}\right) \mathbf{e}+\ldots+e_{1} . .\left(1-e_{k-1}\right) e_{k} \mathbf{e}+ \\
& +e_{1} \ldots\left(1-e_{k-1}\right)\left(1-e_{k}\right) \mathbf{e}+\ldots+\left(1-e_{1}\right) \ldots\left(1-e_{k}\right) \mathbf{e}
\end{aligned}
$$

onde $e_{1} \ldots e_{k}$ são $k$-idempotentes nas condições previamente citadas. Ou seja, é natural que, ao estudar idempotentes primitivos em $\mathbb{F} G \mathbf{e} \cong M_{n}\left(\mathbb{F}_{q}\right)$ encontremos um grande número de idempotentes da forma $e_{1}^{ \pm} \ldots e_{k}^{ \pm}$.

Em $\mathbb{F} G$, se $H$ é um subgrupo de $G$, sendo $\widehat{H}$ um idempotente da álgebra de grupo, portanto, é imediato pensar em idempotentes da forma $\widehat{H}_{i}$, para cada $H_{i}$ subgrupo de $G$,como candidatos aos idempotentes $e_{1}, \ldots, e_{k}$ da decomposição acima. Vale ressaltar que se tomarmos apenas os subgrupos normais de $G$ como idempotentes, então recairemos em uma decomposição da unidade em termos de idempotentes centrais ortogonais. Tais idempotentes mostram-se de extremo valor na descrição dos idempotentes centrais primitivos, tanto em $\mathbb{F} G$ quanto em $\mathbb{Q} G$. 
Notem que para o caso em que $H_{1} \leq H_{2}$, então $\widehat{H}_{1} \cdot \widehat{H}_{2}=\widehat{H}_{2}$. Portanto, se

$$
e_{1}=\widehat{H}_{1}, . ., e_{k}=\widehat{H}_{k} \text { e } H_{1} \leq \ldots \leq H_{k}
$$

então teremos:

$$
\begin{aligned}
e=1 e= & e_{1} . . e_{k} e+e_{1} \ldots\left(1-e_{k}\right) e+\ldots+e_{1} . .\left(1-e_{k-1}\right) e_{k} e+ \\
& +e_{1} \ldots\left(1-e_{k-1}\right)\left(1-e_{k}\right) e+\ldots+\left(1-e_{1}\right) \ldots\left(1-e_{k}\right) e \\
= & \widehat{H}_{k} e+\widehat{H}_{k-1}\left(1-\widehat{H}_{k}\right) e+\ldots+\widehat{H}_{k-2}\left(1-\widehat{H}_{k-1}\right) \widehat{H}_{k} e+ \\
+ & \widehat{H}_{k-2}\left(1-\widehat{H}_{k-1}\right)\left(1-\widehat{H}_{k}\right) e+\ldots+\left(1-\widehat{H}_{1}\right) \ldots\left(1-\widehat{H}_{k}\right) e
\end{aligned}
$$

Vamos então, procurar descrever cada um dos idempotentes primitivos de $\mathbb{F} G \mathbf{e} \cong M_{n}\left(\mathbb{F}_{q}\right)$ por meio de um produto da forma $\widehat{H}_{1}^{ \pm} \ldots \widehat{H}_{k}^{ \pm}$.

As próximas definições e construções são fundamentais para o próposito de nossos algoritmos. É importante ressaltar uma característica marcante e que deve ser observada atentamente no estudo de álgebras de grupo por vias computacionais. A complexidade do objeto em questão cresce de forma extremamente acentuada. $\mathrm{Ou}$ seja, duas álgebras de grupo $\mathbb{F}_{q_{1}} G_{1}$ e $\mathbb{F}_{q_{2}} G_{2}$ tais que $q_{1}$ e $q_{2}$ sejam números próximos bem como $G_{1}$ e $G_{2}$ sejam grupos com ordens similares, o número de elementos das álgebras de grupo podem ser enormemente distantes. Como por exemplo $\mathbb{F}_{3} C_{8}$ e $\mathbb{F}_{5} C_{9}$ enquanto o primeiro possui 6.561 elementos o segundo possui cerca de 2 milhões. Para classes de grupo cuja ordem tenha um crescimento mais acentuado como em $S_{n}$ o tamanho da álgebra de grupo pode ser completamente incomparável. Enquant $\mathbb{F}_{5} S_{3}$ possui 15.625 elementos, $\mathbb{F}_{5} S_{4}$ possui $5,9.10^{16}$ elementos e $\mathbb{F}_{7} S_{5}$ possui $2,6.10^{101}$ elementos. Portanto, devemos pensar de forma muito cautelosa nas estratégias adotadas.

A seguir, definiremos algumas funções, métodos e algoritmos menores que serão utilizados pelo núcleo central do algoritmo principal. Serão eles:

- Analisadores de dimensão:

permitirão a identificação de elementos que comporão os idempotentes primitivos

Compõe os analisadores de dimensão os algoritmos ProgTest advindo da função de dimensão e o algoritmo DimHate.

- Crivo de idempotentes:

Função que eliminará idempotentes identificados como não adequados para 
a composição dos idempotentes primitivos. Será a função $C_{r}: \mathbb{Z}^{i} \rightarrow(\mathbb{F} G)^{m}$ onde $m$ é o número de subgrupos de $G$. As funções do tipo crivo $C_{r}$ darão origem ao algoritmo FiltroCentralizeXemL e DuploCrivo

- Indexadores de idempotentes:

Função que recebe um grupo e relaciona índices inteiros com idempotentes obtidos à partir dos subgrupos do grupo recebido. Será a função $S: \mathbb{Z} \rightarrow \mathbb{F} G$

- Função de Salto:

Função que permite a escolha de outros idempotentes para compor os idempotentes primitivos. Esta função pode levar tanto a endereços distintos quanto a famílias distintas de idempotentes. Funções de salto serão funções do tipo $s_{k}: \mathbb{N} \rightarrow \mathbb{N}^{n}$ e fornecem informações sobre como o algoritmo de busca por idempotentes deve se comportar tomando como base um número natural escolhido pelo usuário.

Definição $2.1 f-$ Endereço: Dada uma função $f: I \subset \mathbb{Z}^{n} \rightarrow(\mathbb{F} G)^{m}$, dizemos que uma família de $m$ elementos $\mathscr{F}$ de $\mathbb{F} G$, (vista como uma $m$-úpla de $(\mathbb{F} G)^{m}$ ) é descrita por um $f-$ Endereço, $\mathscr{A}=\left(k_{1}, . ., k_{n}\right) \in \mathbb{Z}^{n}$ quando $\mathscr{F}=f(\mathscr{A})$.

Definição 2.2 Dado um idempotente primitivo central e, $n$ e $n^{\prime}$ inteiros positivos tais que $n^{\prime} \leq n$ e $\mathbb{F} G \mathbf{e} \cong M_{n}\left(\mathbb{F}_{q}\right)$. Diremos que o conjunto de $f$-endereços

$$
\mathscr{M}_{f}\left(\mathbf{e}, n^{\prime}\right)=\left\{\mathscr{A}_{1} . ., \mathscr{A}_{i}=\left(k_{i_{1}}, \ldots, k_{i_{n^{\prime}}}\right), \ldots\right\}
$$

será um mapa para os idempotentes primitivos (não centrais) de $M_{n}\left(\mathbb{F}_{q}\right)$ com relação à $f$, cujos endereços possuem comprimento máximo $n^{\prime}$ se, por meio de $f$, os elementos $\mathscr{A}$ de $\mathscr{M}$ descrevem um ou mais idempotentes primitivos de $\mathbb{F} G \mathbf{e}$.

Notações: Se $n^{\prime}=n$, então $\mathscr{M}_{f}\left(\mathbf{e}, n^{\prime}\right)=\mathscr{M}_{f}(\mathbf{e})$

$e, n^{\prime}$ ou $f$ podem ser omitidos quando claro no contexo, assim, as seguintes notações representam o mesmo objeto:

$$
\mathscr{M}_{f}\left(\mathbf{e}, n^{\prime}\right)=\mathscr{M}_{f}(\mathbf{e})=\mathscr{M}(\mathbf{e})=\mathscr{M}_{f}=\mathscr{M}
$$

Para encontrar idempotentes de $\mathbb{F} G \mathbf{e}$ descritos por um $f$-Endereço será necessário criar uma função a qual chamaremos de função de salto $s_{k}$ (skip function). A função de salto dependerá de outras funções $\sigma_{i}: \mathbb{N}^{i} \rightarrow \mathbb{N}$. Tal função de salto possui um papel fundamental semelhante ao de ordenação dos idempotentes por meio de indexação dos índices de um $f-$ Endereço $\mathscr{A}$. 
Definição 2.3 (Função de salto)

Sejam $\sigma_{i}: \mathbb{N}^{i} \rightarrow \mathbb{N}$. Definimos a função $s_{k}: \mathbb{N}^{+} \rightarrow \mathbb{Z}^{m}$ como

$$
s_{k}(n)=\left(\sigma_{1}(n), \sigma_{2}\left(\sigma_{1}(n), n\right), \ldots, \sigma_{m}\left(\sigma_{m-1}(n), \ldots, \sigma_{1}(n), n\right)\right)
$$

Exemplo 2.4 Podemos construir uma função de salto $s_{k}(n)$ extremamente elemenar como por exemplo:

$$
s_{k}(n)=(1,1,1 \ldots, 1)
$$

ou então $s_{k}(n)=(n, n, n, n, \ldots, n)$, bastando, por exemplo definir $\sigma_{i}\left(x_{1}, . ., x_{i}\right)=n$.

Funções $s_{k}(n)$ tais que $s_{k}(1)=(1,1 . ., 1)$ serão extremamente úteis futuramente, pois para nosso algoritmo, funções nessas condições serão as funções que encontram famílias de idempotetentes de forma mais rápida.

Diremos que a função $s_{k}(n)=(n, 1, \ldots, 1)$ será a função de salto padrão.

\subsubsection{Analisadores de Dimensão}

Dado um idempotente qualquer $e_{i}$, o seguintes analisadores de dimensão estudam as dimensões de $e_{i} \mathbb{F} G e_{i}$ sobre $\mathbb{F}$.

A seguir apresentamos o algoritmo FdimV2 que transforma a sequência de elementos

$$
e_{i} g_{1} e_{i}, \ldots, e_{i} g_{j} e_{i}, \ldots e_{i} g_{|G|} e_{i}
$$

em uma matriz $M_{|G|}(\mathbb{F})$ e retorna seu posto.

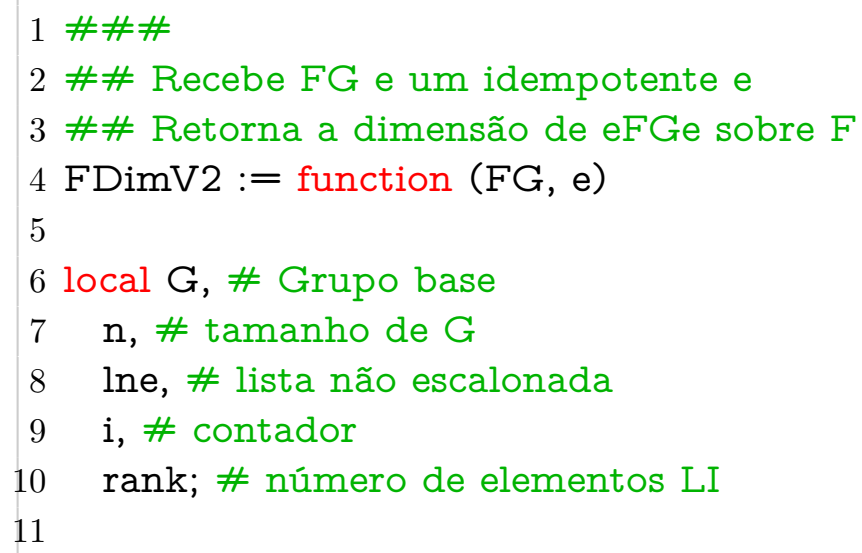


$12 \mathrm{G}:=$ UnderlyingMagma(FG);

$13 \mathrm{n}:=\operatorname{Size}(\mathrm{G})$;

14 lne:= [];

15

16 for $\mathrm{i}$ in $[1 . . \mathrm{n}]$ do

17 Add (lne, CoeficientesByG(FG,G,e*List(G)[i]*e*One(FG)));

18 od;

19

20 rank := RankMat(lne);

21

22 return rank;

23 end;

Definido FDimV2, definiremos um algoritmo excepcionalmente útil que recebe uma lista de idempotentes $e_{1}, . ., e_{k}$ (podendo ser unitária) e retorna as dimensões sobre $\mathbb{F}$ de $e_{1} \mathbb{F} G e_{1}, \ldots, e_{k} \mathbb{F} G e_{k}$. Este será o algoritmo DimHate.

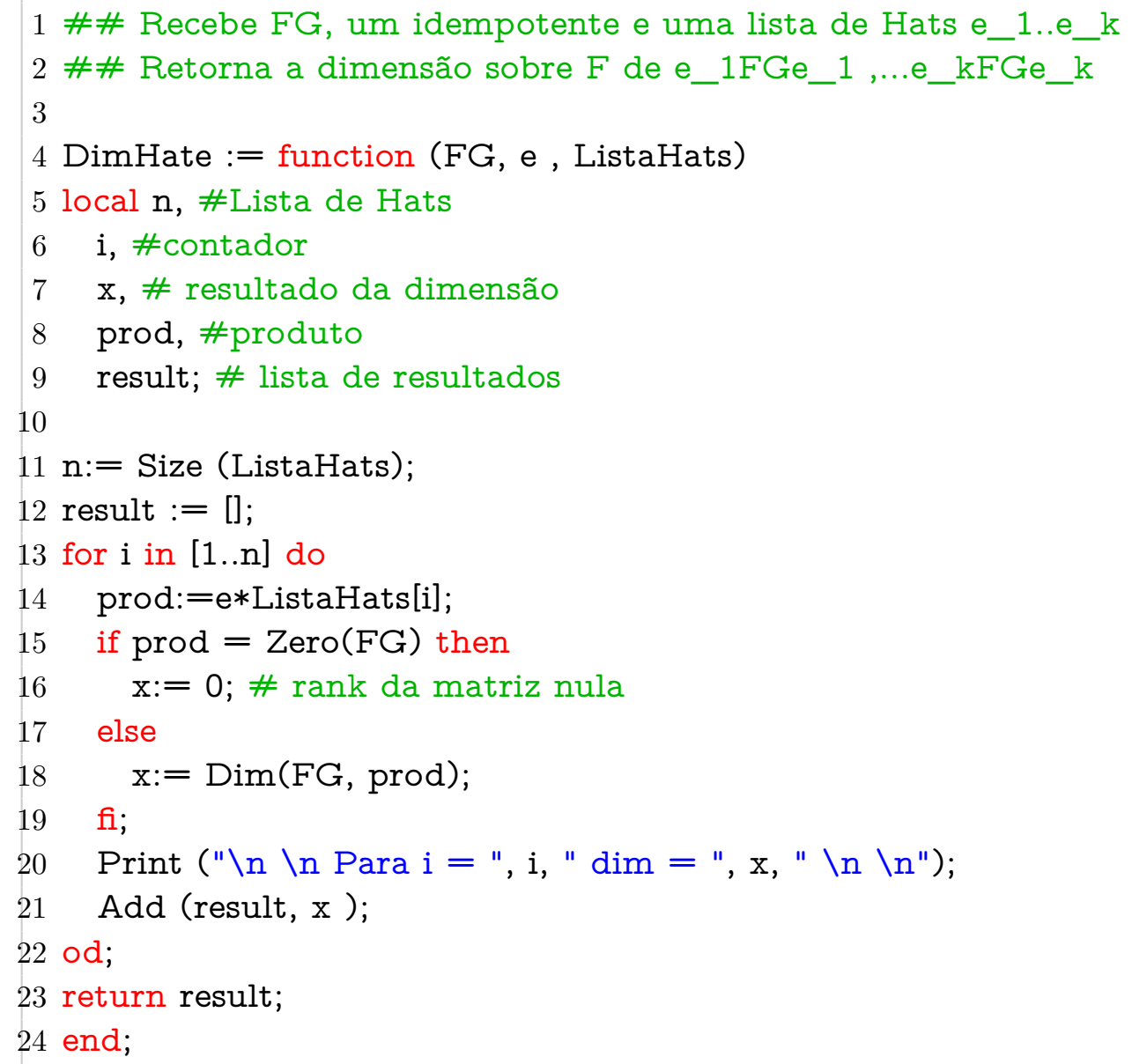


Apesar do analisador anterior ser uma ferramenta poderosa de análise, conforme aumenta-se a ordem de $G$, os elementos de $\mathbb{F} G$ tendem a apresentar suporte cada vez maior, assim o algoritmo pode apresentar problemas de desempelho. Uma estratégia extremamente veloz para trabalhar nas componentes $M_{n}\left(\mathbb{F}_{p^{k}}\right)$ para o caso em que a ordem de $G$ é relativamente grande enquanto a $k$ seja relativamente pequeno é a de utilizar a função de dimensão:

Seja e $\in \mathbb{F} G$ tal que $\mathbb{F} G \mathbf{e} \cong M_{n}\left(\mathbb{F}_{p^{k}}\right)$ e seja $e$ um idempotente qualquer

Considere as funções $g_{G}^{e, i}$ :

$$
\begin{array}{cl}
g_{G}^{e, i}:\left\{g_{1}, . ., g_{i}\right\} \subset G & \rightarrow \mathbb{F} G \\
g & \rightarrow \text { egee }
\end{array}
$$

Temos que para cada $i$ entre 1 e $|G|-1$

$$
\operatorname{dim}_{\mathbb{F}}\left[\operatorname{Im} g_{G}^{e, i}\right] \leq \operatorname{dim}_{\mathbb{F}}\left[\operatorname{Im} g_{G}^{e, i+1}\right]
$$

e como

$$
\operatorname{dim}_{\mathbb{F}}\left[\operatorname{Img}_{G}^{e,|G|}\right]=\operatorname{dim}_{\mathbb{F}} e \mathbb{F} G e \mathbf{e}
$$

segue que

$$
\operatorname{dim}_{\mathbb{F}}\left[I m g_{G}^{e, i}\right] \leq \operatorname{dim}_{\mathbb{F}} e \mathbb{F} G e \mathbf{e}
$$

Considere também as funções $f_{0}$, e $\left\{f_{i}\right\}_{i=1 . .|G|}$, com $f_{0}: \mathbb{F} G \rightarrow\{0\}$ e, sendo $h$ é uma função qualquer com imagem em $\mathbb{F} G$, para cada $i=1 \ldots|G|$, definimos

$$
\begin{aligned}
f_{i}^{h}: \mathbb{F} \times I m h & \rightarrow \mathbb{F} G \\
(\alpha, \beta) & \rightarrow \alpha e g_{i} e \mathbf{e}+\beta
\end{aligned}
$$

Note que dessa forma, temos

$$
\{0\}=\operatorname{Im} f_{0} \subseteq \operatorname{Im} f_{1}^{f_{0}} \subseteq \ldots \operatorname{Im} f_{j}^{f_{j-1}} \subseteq \ldots \subseteq \operatorname{Im} f_{|G|}^{f_{|G|-1}} \subseteq e \mathbb{F} G e \mathbf{e}
$$

então, se $n_{0}$ denota um inteiro positivo qualquer, temos que

$$
\operatorname{dim}_{\mathbb{F}} e \mathbb{F} G e \mathbf{e}=n_{0} \Longleftrightarrow \operatorname{dim}_{\mathbb{F}} \operatorname{Im} f_{i}^{f_{i-1}} \leq n_{0}, i=1, . .,|G|
$$


Assim, na sequência, cada $f_{i}^{f_{i-1}}$, determina por sua imagem, uma quota inferior para $\operatorname{dim}_{\mathbb{F}} e \mathbb{F} G e \mathbf{e}$ enquanto $\operatorname{dim}_{\mathbb{F}}\left[\operatorname{Img}_{G}^{e,|G|}\right]=\operatorname{dim}_{\mathbb{F}} e \mathbb{F} G e \mathbf{e}$.

Definição 2.5 Seja $G$ um grupo finito tal que $|G|=n, \mathbb{F}$ corpo finito, e um idempotente primitivo central de $\mathbb{F} G$ e $e$ um idempotente qualquer de $\mathbb{F} G$. Definimos a função de dimens ão $F^{e}$ com relação à $e$ e as funções de comparação $C_{i}$ as funções definidas por:

$$
F^{e}:\{0, \ldots|G|\} \rightarrow \mathbb{N}
$$

dada por

$$
F^{e}(n)= \begin{cases}F^{e}(n-1)+1 & \text { se } \operatorname{Im} g_{G}^{e, n} \nsubseteq \operatorname{Im} C_{n-1} \\ F^{e}(n-1) & \text { se } \operatorname{Im} g_{G}^{e, n} \subseteq \operatorname{Im} C_{n-1} \\ 0 & \text { se } n=0\end{cases}
$$

onde as funções $C_{n}$ são dadas por

$$
C_{n}= \begin{cases}f_{n}^{C_{n-1}} & \text { se } F^{e}(n)=F^{e}(n-1)+1 \\ C_{n-1} & \text { se } F^{e}(n)=F^{e}(n-1) \\ f_{0} & \text { se } n=0\end{cases}
$$

Da forma como definida, $F^{e}$ é claramente monótona e $F^{e}(|G|)=\operatorname{dim}_{\mathbb{F}} e \mathbb{F} G e \mathbf{e}$. Pela monotonicidade da $F^{e}$, a função de dimensão torna-se uma maneira eficaz de determinar se o idempotente $e$ faz com que $\operatorname{dim}_{\mathbb{F}} e \mathbb{F} G e$ e seja minimal.

Suponha conhecida a dimensão de um ideal minimal do tipo $e^{\prime} \mathbb{F} G e^{\prime} \mathbf{e}$, ou seja, se $\mathbb{F} G \mathbf{e} \cong M_{n}\left(\mathbb{F}_{p^{k}}\right)$ com $n_{0}=p^{k}$ então, se existe $n_{1}$ em $D_{F^{e}}$, o domínio de $F^{e}$, tal que $F^{e}\left(n_{1}\right)>n_{o}$ então $\operatorname{dim}_{\mathbb{F}} e \mathbb{F} G e \mathbf{e} \geq n_{o}$. Assim, quando $|G|$ torna-se muito grande, é possível saber que o idempotente $e$ não é conveniente para obter ideais minimais da forma como queremos, sem necessariamente determinar a dimensão $e \mathbb{F} G e \mathbf{e}$.

Tal forma de testar os idempotentes através da função de dimensão é implementado diretamente através do seguinte código fonte, o qual chamaremos de ProgTest

Exemplo 2.6 Considere $G=<a, b \mid a^{5}=b^{4}=1, b^{-1} a b=a^{2}>$. Temos que $G \cong$ $C_{5} \rtimes C_{4}$. Assim,

$$
\mathbb{F}_{3} G \cong \mathbb{F}_{3} \oplus \mathbb{F}_{3} \oplus \mathbb{F}_{3^{2}} \oplus M_{4}\left(\mathbb{F}_{3}\right)
$$


Sendo $G^{\prime}=\widehat{a}$, então, tomemos $\mathbf{e}=1-\widehat{a}$. Consideremos também fixada a seguinte ordem em $G$ :

$$
\begin{aligned}
& \left\{I d, a, a^{-1}, b, b^{-1}, a^{2}, a b, a b^{-1}, a^{-2}, a^{-1} b, a^{-1} b^{-1}\right. \\
& \left.b a, b a^{-1}, b^{2}, b^{-1} a, b^{-1} a^{-1}, a b^{2}, a^{-1} b^{2}, b a b, b a^{-1} b\right\}
\end{aligned}
$$

isto é, onde $g_{1}=I d, g_{2}=a, \ldots, g_{20}=b a^{-1} b$. Seja $H_{14}=<g_{14}>=<b^{2}>\mathrm{e}$ então $e=\widehat{H_{14}}$.

Vamos verificar o comportamento da função de dimensão $F^{e}$.

Temos inicialmente que $C_{0}=f_{0}$. e $F(0)=0$.

Vamos encontrar $F^{e}(1)$ :

Como $g_{G}^{e, 1}$ é tal que

$$
\begin{array}{cc}
g_{G}^{e, 1}:\left\{g_{1}\right\} \subset G & \rightarrow \mathbb{F} G \\
g & \rightarrow \text { egee }
\end{array}
$$

Temos que $I m g_{G}^{e, 1}=\left\{e g_{1} e \mathbf{e}\right\}=\{e \mathbf{e}\} \nsubseteq \operatorname{Im} C_{0}=\{0\}, \operatorname{Logo}, F(1)=F(0)+1=$ 1.

Agora, para encontrar $C_{1}$, verifica-se que $F(1)=F(0)+1$ portanto $C_{1}=f_{1}^{C_{0}}$, ou seja,

$$
\begin{aligned}
f_{1}^{C_{0}}: \mathbb{F} \times I m C_{0} & \rightarrow \mathbb{F} G \\
(\alpha, \beta) & \rightarrow \alpha e g_{1} e \mathbf{e}+\beta
\end{aligned}
$$

Ou seja $\operatorname{Im}_{1}^{C_{0}}=\left\{0 e g_{1} e \mathbf{e}+0,1 e g_{1} e \mathbf{e}+0,2 e g_{1} e \mathbf{e}+0\right\}=\{0, e \mathbf{e}, 2 e \mathbf{e}\}$.

Assim, $\operatorname{Im} C_{1}=\{0, e \mathbf{e}, 2 e \mathbf{e}\}$.

Vamos agora encontrar $F^{e}(2)$ :

Como $g_{G}^{e, 2}$ é tal que

$$
\begin{array}{cl}
g_{G}^{e, 2}:\left\{g_{1}, g_{2}\right\} \subset G & \rightarrow \mathbb{F} G \\
g & \rightarrow \text { egee }
\end{array}
$$

Claro que $\operatorname{Im} g_{G}^{e, 2}=\left\{0, e \mathbf{e}, e g_{2} e \mathbf{e}\right\} \nsubseteq \operatorname{Im} C_{1}$.Portanto $F^{e}(2)=F(1)+1=2$. 
Note que se o intúito é averiguar se o idempotente $e$ é tal que $\operatorname{dim}_{\mathbb{F}_{3}} e \mathbb{F}_{3} G e \mathbf{e}=1$, já teríamos uma resposta negativa. pois $F^{e}(2)=2>1$.

Para calcular $F^{e}(3)$, temos que $C_{2}=f_{2}^{C_{1}}$, ou seja,

$$
\begin{aligned}
f_{2}^{C_{1}}: \mathbb{F} \times I m C_{1} & \rightarrow \mathbb{F} G \\
(\alpha, \beta) & \rightarrow \alpha e g_{2} e \mathbf{e}+\beta
\end{aligned}
$$

Portanto,

$$
\begin{aligned}
\operatorname{Im} f_{2}^{C_{1}}= & \left\{\alpha e g_{2} e \mathbf{e}+\beta \mid \alpha \in \mathbb{F}_{3}, \beta \in C_{1}\right\} \\
= & \left\{0 e g_{2} e \mathbf{e}+\beta, 1 e g_{2} e \mathbf{e}+\beta, 2 e g_{2} e \mathbf{e}+\beta \mid \beta \in C_{1}\right\} \\
= & \left\{0 e g_{2} e \mathbf{e}+0,1 e g_{2} e \mathbf{e}+0,2 e g_{2} e \mathbf{e}+0,0 e g_{2} e \mathbf{e}+e \mathbf{e},\right. \\
& 1 e g_{2} e \mathbf{e}+e \mathbf{e}, 2 e g_{2} e \mathbf{e}+e \mathbf{e}, 0 e g_{2} e \mathbf{e}+2 e \mathbf{e} \\
& \left.1 e g_{2} e \mathbf{e}+2 e \mathbf{e}, 2 e g_{2} e \mathbf{e}+2 e \mathbf{e}\right\}
\end{aligned}
$$

Dessa forma,

$$
\begin{gathered}
I m C_{2}=\left\{0, e g_{2} e \mathbf{e}, 2 e g_{2} e \mathbf{e}, e \mathbf{e}, e g_{2} e \mathbf{e}+e \mathbf{e}, 2 e g_{2} e \mathbf{e}+e \mathbf{e}\right. \\
\left.2 e \mathbf{e}, e g_{2} e \mathbf{e}+2 e \mathbf{e}, 2 e g_{2} e \mathbf{e}+2 e \mathbf{e}\right\}
\end{gathered}
$$

Porem, neste caso $\operatorname{Im} g_{G}^{e, 3} \subseteq \operatorname{Im} C_{2}$. Logo $F(3)=F(2)=2$.

Continuando o processo, pode-se verificar que $F^{e}$ possui o seguinte comportamento:

\begin{tabular}{|c|c|c|c|c|c|c|c|c|c|c|}
\hline $\mathrm{n}$ & 0 & 1 & 2 & 3 & 4 & 5 & 6 & $\ldots$ & 19 & 20 \\
\hline \hline$F^{e}(n)$ & 0 & 1 & 2 & 2 & 3 & 4 & 4 & $\ldots$ & 4 & 4 \\
\hline
\end{tabular}

1 \#\#\#\#\#\# ProgTest - Teste Progressivo de Dimensões

2 \#\#\# Recebe: FG, um idempotente e, e opcionalmente uma dimensão

3 \#\#\# retorna a própria dimensão em caso positivo

4 \#\#\# retorna 0 em caso negativo

5 \#\#\# caso não fornecido dim, retorna a dimensão encontrada

6

7 ProgTest := function ( FG, e, dim... )

8 local

9 listatempF, \# lista temporária F 
10 listatempS, \# lista temporária Somas linear combin.

11 listadecomp,

12 G , \# grupo base de FG como lista

13 F, \# corpo F em FG como lista

14 dima,\# dimensão atual

15 prodg, \#produto de prod. Gi. prod

16 dimp, \# dimensão procurada

$17 \mathrm{j}$; \#contador

18

19 \#dipensa do teste para $\mathrm{e}=0$ :

20 if $\mathrm{e}=$ Zero(FG) then

21 return 0 ;

22 fi;

$23 \mathrm{G}:=$ List(UnderlyingMagma(FG)); \# G como lista

$24 \mathrm{~F}:=$ List(LeftActingDomain(FG)); \# F como lista

25

26 if Length $(\operatorname{dim})=1$ then

$27 \operatorname{dimp}:=\operatorname{dim}[1]$

28 else

29 dimp:= Size(G); \# nunca será maior que $G$

30 fi;

31

32 dima:=0;

33 listatempF:=[]; \# lista temporária F

34 listatempS:=[]; \# lista temporária Somas linear combin.

35 listadecomp :=[Zero(FG)];

$36 \mathrm{j}:=1$;

37

38 while $\mathrm{j}<=\operatorname{Size}(G)$ do \# inicio do teste de dimensão

39 prodg: $=e * G[j] * e$;

40 if not(prodg) in listadecomp then

41 dima:=dima+1; \# dim atual.

42 Print ("\n dima=", dima, "mas $\operatorname{dimp}=$ ", $\operatorname{dimp}$ );

43 Print ("\n Teste:", dima > dimp );

44 if dima > dimp then

$45 \quad \mathrm{j}:=\operatorname{Size}(G)+1 ;$ \#sai do loop;

46 dima $:=0$;

47 Print (" \n Zerou dima dima = ", dima );

48 return dima;

49 else 


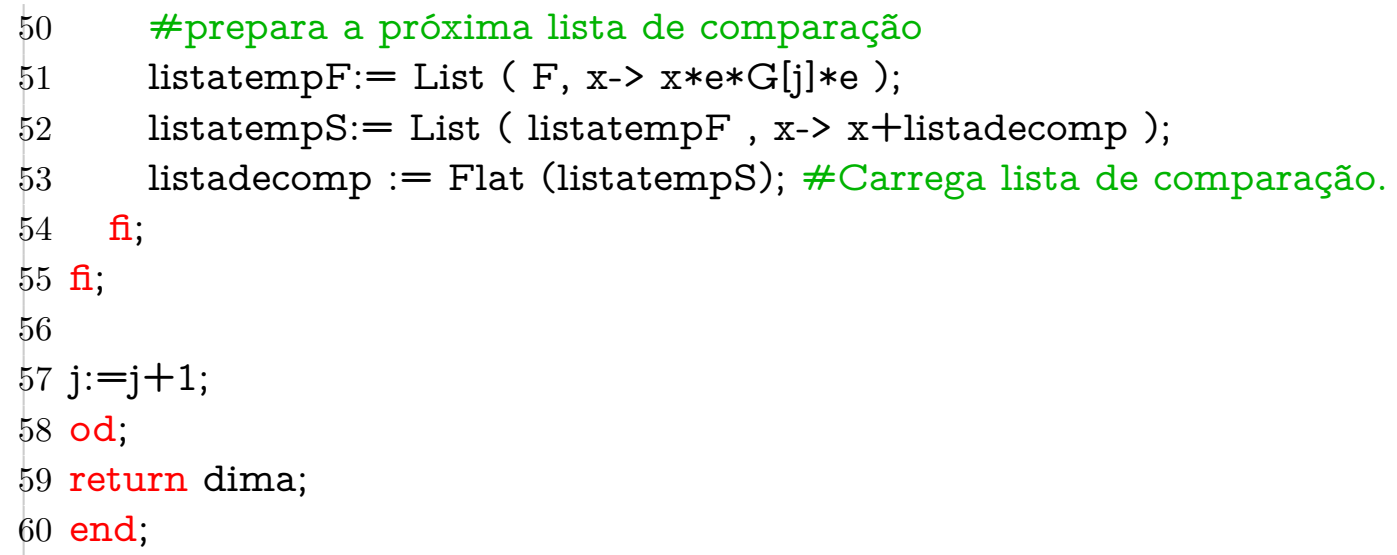

\subsubsection{Funções de Crivo}

Prosseguindo com os elementos fundamentais para a construção de nosso algoritmo, será necessário criarmos algumas funções das quais chamaremos funções de crivo. As funções de crivo exercem exclusivamente o papel de selecionar ou filtrar elementos de um conjunto dadas certas condições. Em geral, denotaremos nossas funções de crivo por $C_{r}^{*}$ onde * representará um subtipo de função de crivo. Teremos basicamente três tipos de função de crivo, baseadas em centralizadores, subgrupos (relações de inclusão) e filtro do duplo idempotente, representados respectivamente por $\mathscr{C}, O_{G r}, D_{i d p}$.

Definição 2.7 Seja $L$ um subconjunto de $\mathbb{F} G$ e $x \in \mathbb{F} G$, um elemento qualquer. Definimos a função de crivo com relação ao centralizador $\mathscr{C}(x)=\mathscr{C}_{\mathbb{F} G}(x)$, a função $C_{r}^{\mathscr{C}(x)}$ dada por:

$$
\begin{aligned}
C_{r}^{\mathscr{C}(x)}: L \subset \mathbb{F} G & \longrightarrow \mathbb{F} G \\
\alpha & \longrightarrow \begin{cases}\alpha & \text { se } \alpha \in \mathscr{C}_{\mathbb{F} G}(x) \\
0 & \text { se } \alpha \notin \mathscr{C}_{\mathbb{F} G}(x)\end{cases}
\end{aligned}
$$

Dessa forma, $\operatorname{Im} C_{r}^{\mathscr{C}(x)} \backslash\{0\}$ corresponde aos elementos não nulos de $L$ e comutam com $x$ em $\mathbb{F} G$. Escreveremos apenas $C_{r}^{\mathscr{C}}$ quando nos referirmos à este tipo de função de crivo independentemente do elemento $x$.

Em nosso contexto, utilizaremos no lugar do elemento $x$ um idempotente $e$ e no lugar de $L$ uma lista contendo idempotentes previamente calculados. Dessa forma, garatiremos a criação de novos idempotentes através da imagem de $C_{r}^{\mathscr{C}(e)}$ uma vez que se $l \in L$ é idempotente e $l \in \operatorname{Im} C_{r}^{\mathscr{C}(x)}$, então le também será um idempotente de $\mathbb{F} G$. 
O segundo tipo de filtro que nos será útil será uma função extremamente simples porém de extrema utilidade. Dado um idempotente $e$ do tipo $e=(1-\widehat{H}) \cdot e^{\prime}$, se $H$ é subgrupo de $H_{2}$, então o produto $\widehat{H}_{2} \cdot e=\widehat{H}_{2}(1-\widehat{H}) e^{\prime}=\left(\widehat{H}_{2}-\widehat{H}_{2} \widehat{H}\right) e^{\prime}=$ $\left(\widehat{H}_{2}-\widehat{H}_{2}\right) e^{\prime}=0$. Dessa forma, o produto $\widehat{H}_{2} e$ não nos fornece um novo idempotente não nulo, assim, torna-se importante ter controle sobre este caso.

Definição 2.8 Sejam $H$ um subgrupo de $G$ e $S u b G$ o conjunto de todos os seus subgrupos. Definimos a função de crivo dada pela relação $\geq$, a função $C_{r}^{O_{G r}}$ dada por:

$$
\begin{aligned}
C_{r}^{O_{G r}(H)}: S u b G & \longrightarrow \mathbb{N} \\
X & \longrightarrow \begin{cases}1 & \text { se } X \geq H \\
0 & \text { casocontrário }\end{cases}
\end{aligned}
$$

Ou seja, dado um subgrupo $X$ de $G, C_{r}^{O_{G r}(H)}(X)$ retorna 1 caso $X$ seja um subgupo de $G$ que contém $H$ (overgroup) ou 0 caso contrário. Para não sobrecarregar a notação, se $H$ estiver claro no contexto, ou se nos referirmos a esta função sem mencionar $H$, denotaremos apenas por $C_{r}^{O_{G r}}$.

Por fim, apresentamos nossa última função de crivo, a função $C_{r}^{D_{i d p}}$. O rótulo $D_{i d p}$ faz menção ao termo duplo idempotente. O propósito deste filtro é, com ele, criar idempotentes à partir de dois outros elementos mantendo a ortogonalidade com demais idempotentes previamente calculados. Seja $b \in \mathbb{F} G$ um idempotente, se $a \in \mathbb{F} G$ é um elemento tal que $a b$ seja idempotente então $b a b$ também será idempotente. De fato:

$$
\begin{aligned}
(b a b)^{2} & =(b a b)(b a b)=b a b b a b= \\
& =b a b^{2} a b=b a b a b=b(a b)^{2} \\
& =b a b
\end{aligned}
$$

Além disso, se $b$ é ortogonal (à esquerda e à direta) a um terceiro idempotente $e^{\prime}$, então o novo idempotente $e=b a b$ é tal que

$$
\begin{aligned}
& e \cdot e^{\prime}=(b a b) e^{\prime}=(b a)\left(b e^{\prime}\right)=0 \\
& e^{\prime} \cdot e=e^{\prime}(b a b)=\left(e^{\prime} b\right)(a b)=0
\end{aligned}
$$

Assim, temos um crivo que cumprirá função semelhante ao crivo $C_{r}^{\mathscr{C}}$ porém livre de algumas condições presentes durante o uso de $C_{r}^{\mathscr{C}}$. Para criarmos idempotentes novos à partir de $C_{r}^{\mathscr{C}}$, precisamos partir de uma lista prévia de idempotentes $L$. Já, para $C_{r}^{D_{i d p}}$ poderemos partir de uma lista $L$ qualquer.

Considerações serão feitas com relação ao uso dessas duas funções. 
Definição 2.9 Seja $L$ um subconjunto de $\mathbb{F} G$ e $e \in \mathbb{F} G$, um idempotente. Definimos a função de crivo com relação à dupla idempotência, a função $C_{r}^{D_{i d p}(e)}$ dada por:

$$
\begin{aligned}
C_{r}^{I d p(e)}: L \subset \mathbb{F} G & \longrightarrow \mathbb{F} G \\
\alpha & \longrightarrow \begin{cases}\alpha & \text { se } \alpha e \text { éidempotente } \\
0 & \text { casocontrário }\end{cases}
\end{aligned}
$$

Notemos que $L$ é uma lista de idempotente, a imagem de $C_{r}^{\mathscr{C}}$ é estável por $C_{r}^{I d p(e)}$. Ou seja,

$$
C_{r}^{I d p(e)}\left(C_{r}^{\mathscr{C}}(L)\right)=C_{r}^{\mathscr{C}}(L)
$$

Além disso,

$$
C_{r}^{\mathscr{C}(e)}(L) \subset C_{r}^{I d p(e)}(L)
$$

É importante ressaltar que a troca de $C_{r}^{\mathscr{C}(e)}(L)$ por $C_{r}^{I d p(e)}(L)$ pode levar a um número maior de famílias de idempotentes. Pode entretanto, haver uma diferença de desempenho.

\subsubsection{A Construção de um HatMap}

Nossas peças principais para a construção de idempotentes primitivos serãos idempotentes dados por hats, ou seja, idempotentes do tipo $\widehat{H}$ ou por $(1-\widehat{H})$ onde $H$ é um subgrupo de $G$. Definiremos agora o que é um HatMap, ou seja, um mapa dado por hats, diremos como construi-lo e sua relevância para finalmente exibirmos nosso principal algoritmo de busca por idempotentes.

Considere um conjunto $S$ de idempotentes indexado por $s: \mathbb{Z} \rightarrow S$, com $s_{i} \stackrel{\text { def }}{=} s(i)$

Sejam $\xi^{m}: \mathbb{Z}^{m} \rightarrow \mathbb{F} G$ funções que associam a cada endereço $\mathscr{A} \in \mathscr{M}(\mathbf{e}, m)=$ $\mathscr{M}$ um idempotente de $\mathbb{F} G \mathbf{e}$ definidas por:

$$
\begin{array}{ccc}
\xi^{i}: & \mathscr{M}(\mathbf{e}, i) & \mathbb{F} G \\
\mathscr{A}=\left(k_{1}, . ., k_{i}\right) & \rightarrow & s_{k_{i}}\left(1-\sum_{j=1}^{i-1} \xi\left(k_{j}\right)\right) \mathbf{e}
\end{array}
$$

para $1<i \leq m$ e para $i=1$ defina $\xi^{1}\left(k_{1}\right)=s_{k_{1}} \mathbf{e}$.

Definindo $\left.\mathscr{A}\right|_{j}$ como o endereço $\mathscr{A}$ restrito à $j$ - ésima posição, ou seja, se 
$\mathscr{A}=\left[a_{1, . .}, a_{j}, a_{j+1}, \ldots a_{m}\right]$, então $\left.\mathscr{A}\right|_{j}=\left[a_{1}, . ., a_{j}\right]$, e sendo

$$
M=\left\{\left.\mathscr{A}\right|_{j}, \operatorname{com} \mathscr{A} \in \mathscr{M}, 1 \leq j \leq m\right\}
$$

Definimos a função $\xi$ como

$$
\begin{array}{ccc}
\xi: M & \longrightarrow & \mathbb{F} G \mathbf{e} \\
\mathscr{A} & \rightarrow & \xi^{m_{\mathscr{A}}}(\mathscr{A})
\end{array}
$$

onde $m_{\mathscr{A}}$ corresponde ao comprimento do endereço $\mathscr{A}$. Assim, definidas as funções $\xi^{m}$, a função $\xi$ associa um endereço de qualquer tamanho a um idempotente de $\mathbb{F} G \mathbf{e}$.

Particularmente nos interessará a situação em que $S=\{\widehat{H},(1-\widehat{H})\}_{H \in S u b G}$, neste caso indexaremos $S$ por $s$ da seguinte forma:

$$
s(i)= \begin{cases}\widehat{H}_{i} & \text { se } i>0 \\ 1-\widehat{H}_{i} & \text { se } i<0 \\ 0 & \text { se } i=0\end{cases}
$$

Considere $S$ como definido acima, as funções $\xi$ e $s$ como definidas acima para um e $\in \mathbb{F} G$, e $n$ tal que $\mathbb{F} G \mathbf{e} \cong M_{n}\left(\mathbb{F}_{q}\right)$. Vamos contruir um mapa de idempotentes de $\mathbb{F} G$ e o qual chamaremos de HatMap. Ou seja, vamos descrever uma série de idempotentes de $\mathbb{F} G \mathbf{e}$ utilizando $\Xi-$ endereços $\mathscr{A}$ tais que

$$
\begin{aligned}
& \Xi_{i}: \quad \mathscr{M}(\mathbf{e}, i) \quad \longrightarrow \quad(\mathbb{F} G)^{i} \\
& \mathscr{A}=\left(k_{1}, . k_{i}\right) \quad \rightarrow \quad\left(\xi\left(k_{1}\right), \xi\left(k_{1}, k_{2}\right), \ldots, \xi\left(k_{1}, . ., k_{i}\right)\right)
\end{aligned}
$$

E da mesma forma, definimos

$$
\begin{array}{lccc}
\Xi: & M & (\mathbb{F} G)^{i} \\
& \mathscr{A}=\left(k_{1},, . k_{i}\right) & \rightarrow & \left(\xi\left(k_{1}\right), \xi\left(k_{1}, k_{2}\right), \ldots, \xi\left(k_{1}, . ., k_{i}\right)\right)
\end{array}
$$

Ou seja, dado um endereço $\mathscr{A}$ de comprimento qualquer $i, \xi(\mathscr{A})$ é um idempotente de $\mathbb{F} G \mathbf{e}$ e $\Xi(\mathscr{A})$ corresponde a uma família de idempotentes de $\mathbb{F} G \mathbf{e}$ dada por uma $i$ - úpla de $\mathbb{F} G$. Com a função $\Xi$ depende da função $\xi$, diremos também que $\mathscr{A}$ são $\xi$ - endereços.

A construção de um HatMap deve ser feita camada a camada, portanto, vamos trabalhar com um $n^{\prime} \leq n$ de cada vez. Assim, determinaremos nossos Layers $L_{n^{\prime}}$, ou seja, endereços incompletos de comprimento $n^{\prime}$. Consideremos primeiro o mapa 
formado por endereços de comprimento 1:

$$
\mathscr{M}_{\mathcal{S}}(\mathbf{e}, 1)=\left\{a_{1}^{L_{1}}, \ldots, a_{i}^{L_{1}}=\left(k_{i_{1}}\right), \ldots\right\}
$$

Aqui, $L_{1}$ refere-se a Layer 1 e cada $k_{i_{1}} \in \operatorname{Im}\left(S^{-1}\right)$ é tal que

$$
s\left(k_{i_{1}}\right) \in \mathscr{C}_{\mathbb{F} G}(\mathbf{e}) \cap S=S
$$

e

$$
\operatorname{dim}_{\mathbb{F}}\left(s\left(a_{k}\right)\right) \mathbb{F} G\left(s\left(a_{k}\right)\right) \mathbf{e}=q,
$$

com $a_{i}>a_{j}$ se $i>j$

Para a construção das demais camadas $L_{n^{\prime}} \operatorname{com} 1<n^{\prime}<n$, queremos criar mapas de comprimento $n^{\prime}$ da forma

$$
\mathscr{M}_{\mathcal{S}}\left(\mathbf{e}, n^{\prime}\right)=\left\{a_{1}^{L_{n^{\prime}}}, \ldots, a_{i}^{L_{n^{\prime}}}=\left(k_{i_{1}}, \ldots, k_{i_{n^{\prime}}}\right), \ldots\right\}
$$

onde cada $a_{i}^{L_{n^{\prime}}}=\left(k_{i_{1}}, \ldots, k_{i_{n^{\prime}}}\right) \in \operatorname{Im}^{n^{\prime}}\left(S^{-1}\right)$, é tal que $\xi\left(a_{i}^{L_{n^{\prime}-1}}\right)$ e $\xi\left(a_{i}^{L_{n^{\prime}}}\right)$ sejam um idempotentes primitivos ortogonais. Para tal, para cada endereço $a_{i}^{L_{n^{\prime}-1}}$ toma-se todos os hats $s\left(k_{i_{n^{\prime}}}\right)$ tais que

$$
s\left(k_{i_{n^{\prime}}}\right) \in \mathscr{C}_{\mathbb{F} G}\left(\xi\left(a_{i}^{L_{n^{\prime}}-1}\right)\right)
$$

$\mathrm{e}$

$$
\operatorname{dim}_{\mathbb{F}} s\left(k_{i_{n^{\prime}}}\right) \xi\left(a_{i}^{L_{n^{\prime}-1}}\right) \mathbb{F} G s\left(k_{i_{n^{\prime}}}\right) \xi\left(a_{i}^{L_{n^{\prime}-1}}\right) \mathbf{e}=q
$$

Assim, se obtivermos $m_{o}$ hats nessas condições, a adjunção de cada um desses elementos ao final da camada $L_{n^{\prime}-1}$ de $a_{i}^{L_{n^{\prime}-1}}$ nos fornecerá $m_{o}$ novos endereços de comprimento $n^{\prime}$. Ou seja, o crescimento do número de endereços cresce multiplicativamente, o que pode acarretar em sérios problemas computacionais.

Um hatmap $\mathscr{M}=\mathscr{M}\left(\mathbf{e}, n^{\prime}\right)$ será dito completo se $n^{\prime}=n$ onde $n$ é tal que $\mathbb{F} G \mathbf{e} \cong$ $M_{n}\left(\mathbb{F}_{p^{k}}\right)$. Ou seja, se para cada $\mathscr{A} \in \mathscr{M}$, temos que $\Xi(\mathscr{A})=\left(e_{1}, . ., e_{n}\right) \in(\mathbb{F} G)^{n}$ corresponde a uma $n$-úpla formada por idempotentes primitivos de $\mathbb{F} G \mathbf{e}$. Para a última camada do HatMap, ou seja, $L_{n}$, há duas formas de preenchê-la.

Podemos preencher seguindo exatamente como nas camadas anteriores, ou podemos simplesmente interromper o processo e adicionar o número 1 na última 
camada (ou $k$, tal que $s^{-1}(k)=1_{\mathbb{F} G}$ ), pois o último idempotente está determinado por $n-1$ idempotentes obtidos. Ou seja, determinados os $n-1$ idempotentes, o não interrompimento do processo leva a uma série de idempotentes redundantes.

Dado um mapa $\mathscr{M}=\mathscr{M}(\mathbf{e}, n)$ completo, obtemos diversas maneiras de escrever famílias completas de idempotentes primitivos de $\mathbb{F} G$ e. Porém, também é possível, para grupos Abelianos, criar Hatmaps utilizando $e=1$ como o faremos em $\mathbb{Q} G$ , onde mapas formados majoriatiamente por subgrupos normais de $G$ são capazes de descrever os idempotentes centrais primitivos de $\mathbb{Q} G$ para um número muito significativamente grande de exemplos.

Vale destacar que adiante definiremos outras funções do tipo $\xi: \mathscr{M} \rightarrow \mathbb{F} G$ e $\Xi: \mathscr{M} \rightarrow(\mathbb{F} G)^{n}$ e , para distingui-las, as funções que acabamos de definir serão também escritas como $\xi_{\mathfrak{h}} \mathrm{e} \Xi_{\mathfrak{h}}$ em referência aos endereços de um Hatmap.

Exemplo 2.10 Considere novamente $\mathbb{F}=\mathbb{F}_{2}$ e $G=G_{[27,4]}$, ou seja:

$$
G=<a, b \mid a^{9}=1, b^{3}=1, b a=a^{4} b>
$$

Sendo

$$
\begin{aligned}
S u b G=[ & <I d_{G}>,<a^{3}>,<b>, \\
& <a^{-1} b a>,<a b a^{-1}>, \\
& <b, a^{3}>,<a>,<b a, b^{-1} a^{-1}>, \\
& \left.<b a^{-1}, b^{-1} a>,<a, b>\right]
\end{aligned}
$$

Se $\mathbf{e}=\left(1-\widehat{H}_{2}\right)$, então $\mathbb{F} G \mathbf{e} \cong M_{3}\left(\mathbb{F}_{4}\right)$. Como a componente simples é isomorfa a uma componente matricial de ordem $3 \times 3$, queremos um Hatmap de comprimento 3. Utilizando $S=\left\{\widehat{H}_{i}\right\}_{H_{i} \in S u b G}$, construimos o seguinte Hatmap :

$$
\begin{aligned}
\mathscr{M}(\mathbf{e}, 3)= & {[[3,4,1],[3,4,5],[3,5,1],} \\
& {[3,5,4],[4,3,1],[4,3,5], } \\
& {[4,5,1],[4,5,3],[5,3,1], } \\
& {[5,3,4],[5,4,1],[5,4,3]] }
\end{aligned}
$$

Uma possível representação visual para o Hatmap anterior pode ser vista na figura 2.1 
42 ALGORITMOS DE BUSCA POR IDEMPOTENTES NÃO CENTRAIS

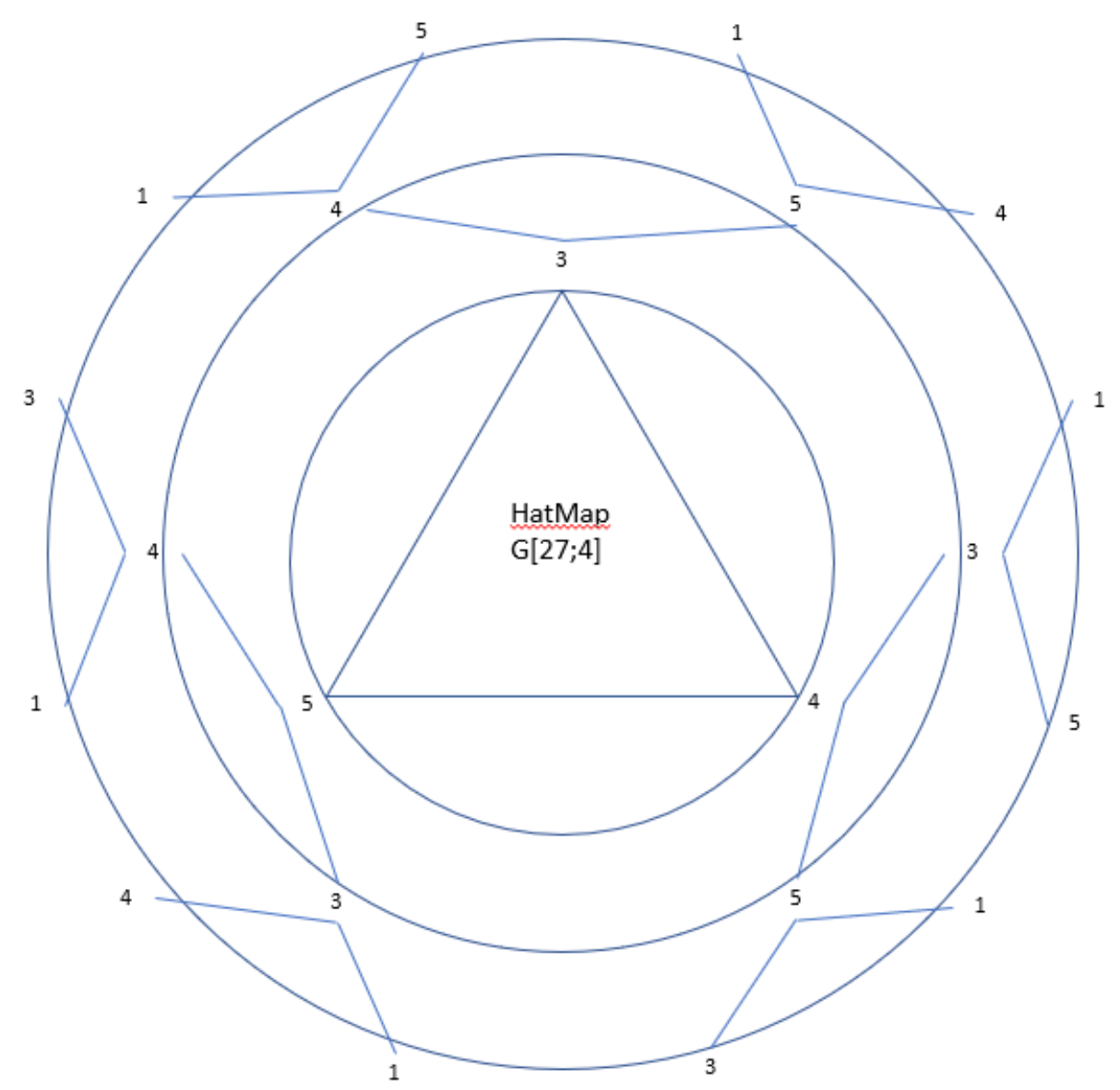

Figura 2.1: Representação gráfica de $\mathscr{M}(\mathbf{e}, 3)$ em $\mathbb{F}_{2} G_{[27,4]}$ 
Podemos, entretanto, possuir grupos de mesma ordem, tais que sobre o mesmo corpo $\mathbb{F}$,o Hatmap seja completamente diferente, conforme o próximo exemplo.

Exemplo 2.11 Considere $\mathbb{F}=\mathbb{F}_{2}$ e $G=G_{[27,3]}$, ou seja:

$$
G=<a, b \mid a^{3}=b^{3}=(b a)^{3}=\left(b a^{-1}\right)^{3}=1>
$$

Sendo

$$
\begin{aligned}
S u b G=[ & <I d_{G}>,<a(a b)^{2} b>,<a>,<b^{-1} a b>,<b a b^{-1}>,<b>, \\
& <a^{-1} b a>,<a b a^{-1}>,<a b>,<b a>,<a^{2} b a^{-1}>,<a^{2} b>, \\
& <a b a>,<a^{3} b a^{-1}>,<a, b a b^{-1}>,<b, a b a^{-1}>, \\
& \left.<b a, b^{-1} a^{-1}>,<b a^{-1}, b^{-1} a>,<a, b>\right]
\end{aligned}
$$

Se $\mathbf{e}=\left(1-\widehat{H}_{2}\right)$, então $\mathbb{F} G \mathbf{e} \cong M_{3}\left(\mathbb{F}_{4}\right)$. Utilizando $S=\left\{\widehat{H}_{i}\right\}_{H_{i} \in S u b G}$,

Construimos o seguinte Hatmap :

$$
\begin{aligned}
\mathscr{M}(\mathbf{e}, 3)= & {[[3,4,1],[3,4,5],[3,5,1],[3,5,4],} \\
& {[4,3,1],[4,3,5],[4,5,1],[4,5,3], } \\
& {[5,3,1],[5,3,4],[5,4,1],[5,4,3], } \\
& {[6,7,1],[6,7,8],[6,8,1],[6,8,7], } \\
& {[7,6,1],[7,6,8],[7,8,1],[7,8,6], } \\
& {[8,6,1],[8,6,7],[8,7,1],[8,7,6], } \\
& {[9,10,1],[9,10,11],[9,11,1], } \\
& {[9,11,10],[10,9,1],[10,9,11], } \\
& {[10,11,1],[10,11,9],[11,9,1], } \\
& {[11,9,10],[11,10,1],[11,10,9], } \\
& {[12,13,1],[12,13,14],[12,14,1], } \\
& {[12,14,13],[13,12,1],[13,12,14], } \\
& {[13,14,1],[13,14,12],[14,12,1], } \\
& {[14,12,13],[14,13,1],[14,13,12]] }
\end{aligned}
$$

Uma possível representação visual para o Hatmap anterior pode ser vista na figura 2.2 


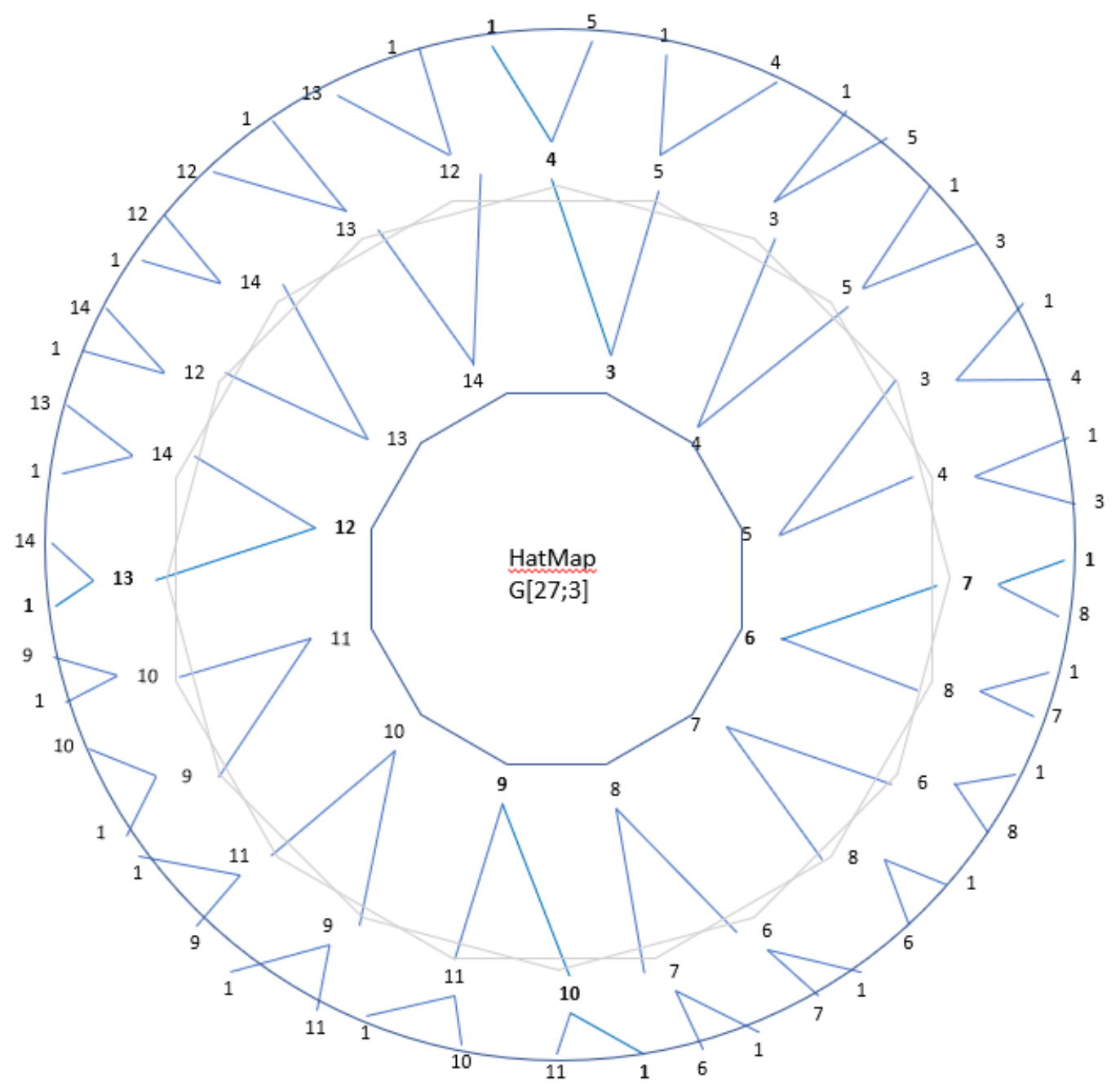

Figura 2.2: Representação gráfica de $\mathscr{M}(\mathbf{e}, 3)$ em $\mathbb{F}_{2} G_{[27,3]}$ 
Sendo $\mathscr{M}_{1}$ o Hatmap obtido no primeiro exemplo, em $\mathbb{F}_{2} G_{[27,4]}$ e $\mathscr{M}_{2}$ o Hatmap obtido no segundo exemplo, em $\mathbb{F}_{2} G_{[27,3]}$. É possível verificar que em $\mathscr{M}_{2}$ existem 4 famílias completamente distintas de idempotentes, 37 famílias distintas e um total de 12 idempotentes primitivos distintos. Além disso, todos esses idempotentes dão origem a códigos lineares com parâmetros $[27,6,6]$.

\subsubsection{O Algoritmo Principal}

A construção de um Hatmap completo pode demandar quantidades enormes de recursos e até mesmo se tornar inviável. Nosso objetivo é obter um endereço $\mathscr{A}$ de $\mathscr{M}$ sem precisar construi-lo por completo. Para tal, vamos proceder de forma similar à construção de um HatMap, porém faremos uso das fu $\sqrt{ }$ nções de crivo $C_{r} \mathrm{e}$ das funções de dimensão $F^{\mathbf{e}}$, peças fundamentais em nosso algoritmo, previamente mencionadas.

Algoritmo 2.12 HatMapXi Sejam $\mathbb{F} G$ uma álgebra de grupo finita, e sejam $\Xi, \xi$ e s como definidas anteriormente com relação a $S=\{\widehat{H}\}_{H \in S u b G}$. Seja e $\in \mathbb{F} G$ um idempotente central primitivo de $\mathbb{F} G$ tal que $\mathbb{F} G \mathbf{e} \cong M_{n}\left(\mathbb{F}_{q}\right)$ com $n, q \in \mathbb{N}$ tal que $\mathscr{M}(e) \neq \emptyset$ sejam $\Xi$-endereços. Dados $s_{k}$, uma função de salto, e $N \in \mathbb{N}$ um natural qualquer, então

$$
\operatorname{HatMapXi}(N, e, F G, s k) \in \mathscr{M}(e)
$$

Sendo seu algoritmo dado pelo seguinte código fonte

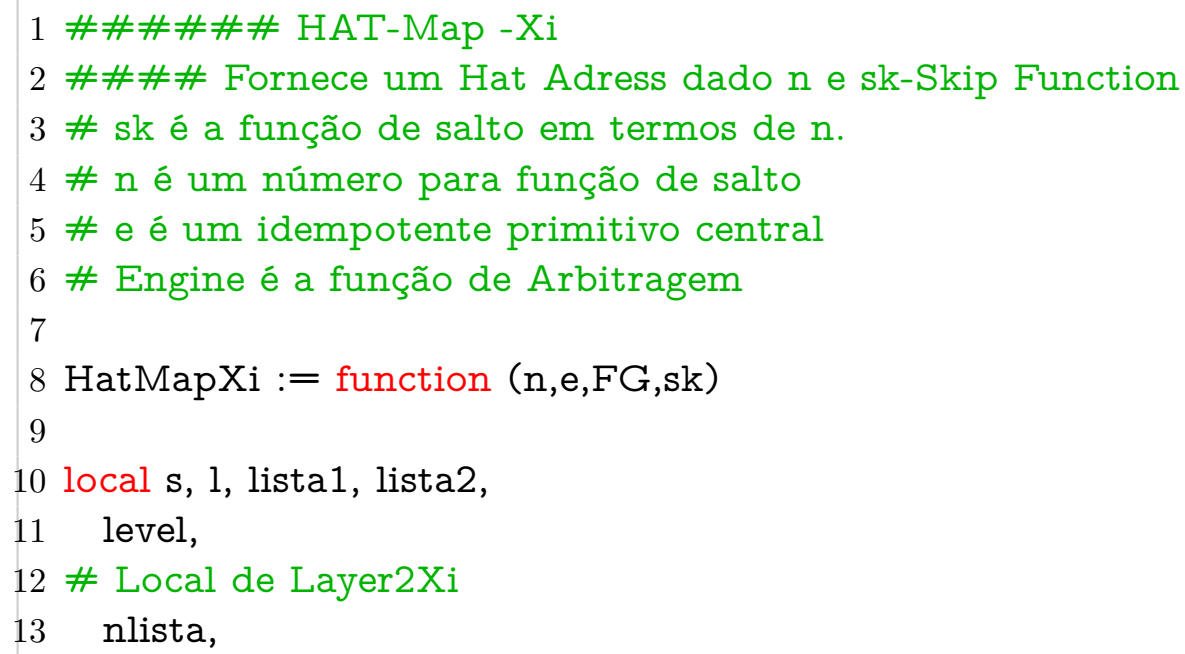


$14 \mathrm{i}, \mathrm{j}, \mathrm{k}, \mathrm{m}$, \#contadores

15 idemp,

16 pos, \#posição dada pelo engine

17 p, \# tamanho da sublista de lista.

18 neg, \# (1-e1-e2..-en)

19 lista2Xi, \#Xi

20 soma, listafin,

21 \# local de LayerAnaliseXi

22 list1LAX, \#list1 de LayerAnaliseXi

23 hatnumberlist, dim,

24 HatMap; \#ResultadoFinal

25

$26 \mathrm{~s}:=$ Engine(2,[1,e,n,FG]); \# recebe os Hats

27 level := Size(sk(n)); \# Número de idempotentes esperado

28

29 \#Calcula primeira camada

$30 \mathrm{l}:=0$;

31

32 hatnumberlist:=Engine (1,[sk(n)[1],e, list1LAX,FG,0]); \# [Camada 1 , dimensão]

33

34 lista2:= hatnumberlist[1];

35 dim:= hatnumberlist[2];

$36 \mathrm{l}:=\mathrm{l}+1$;

37

38 \#\#\#

39 \#\#Cálculo Demais Camadas

40

41 while 1 < level do

$42 \quad$ lista1 := lista2;

43 nlista:= Size(lista1); \#comprimento anterior -> Sempre 1 ?

44 lista2:=[]; \#Esvazia lista2

$45 \quad \mathrm{i}:=1$;

$46 \mathrm{~m}:=$ Size(lista1[i]);

47 idemp:= List([1..m+1]); \# Abre espaço para receber o prox idemp.

48 idemp[1]:= Zero(FG);

49 soma:=Zero(FG);

50 for $\mathrm{k}$ in $[2 . . \mathrm{m}+1]$ do

51 pos:= Engine $(3,[1, \mathrm{e}, \mathrm{n}, \mathrm{FG}])($ lista1[i] $[\mathrm{k}-1])$;

52 idemp $[\mathrm{k}]:=(\mathrm{One}(\mathrm{FG})$-soma $) * \mathrm{~s}[\mathrm{pos}] *(\mathrm{One}(\mathrm{FG})$-soma)*e ; \# 


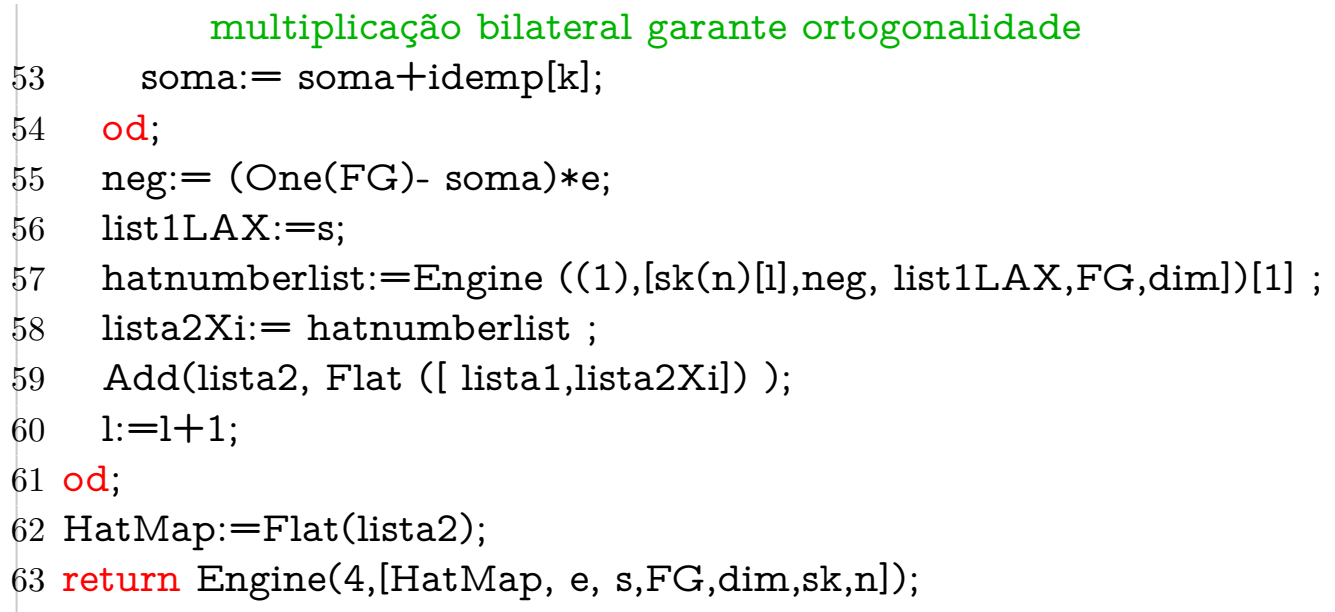

O algoritmo anterior depende de uma série de informações que são fornecidas através de um núcleo externo chamado de Engine neste caso, utilizamos o seguinte código fonte:

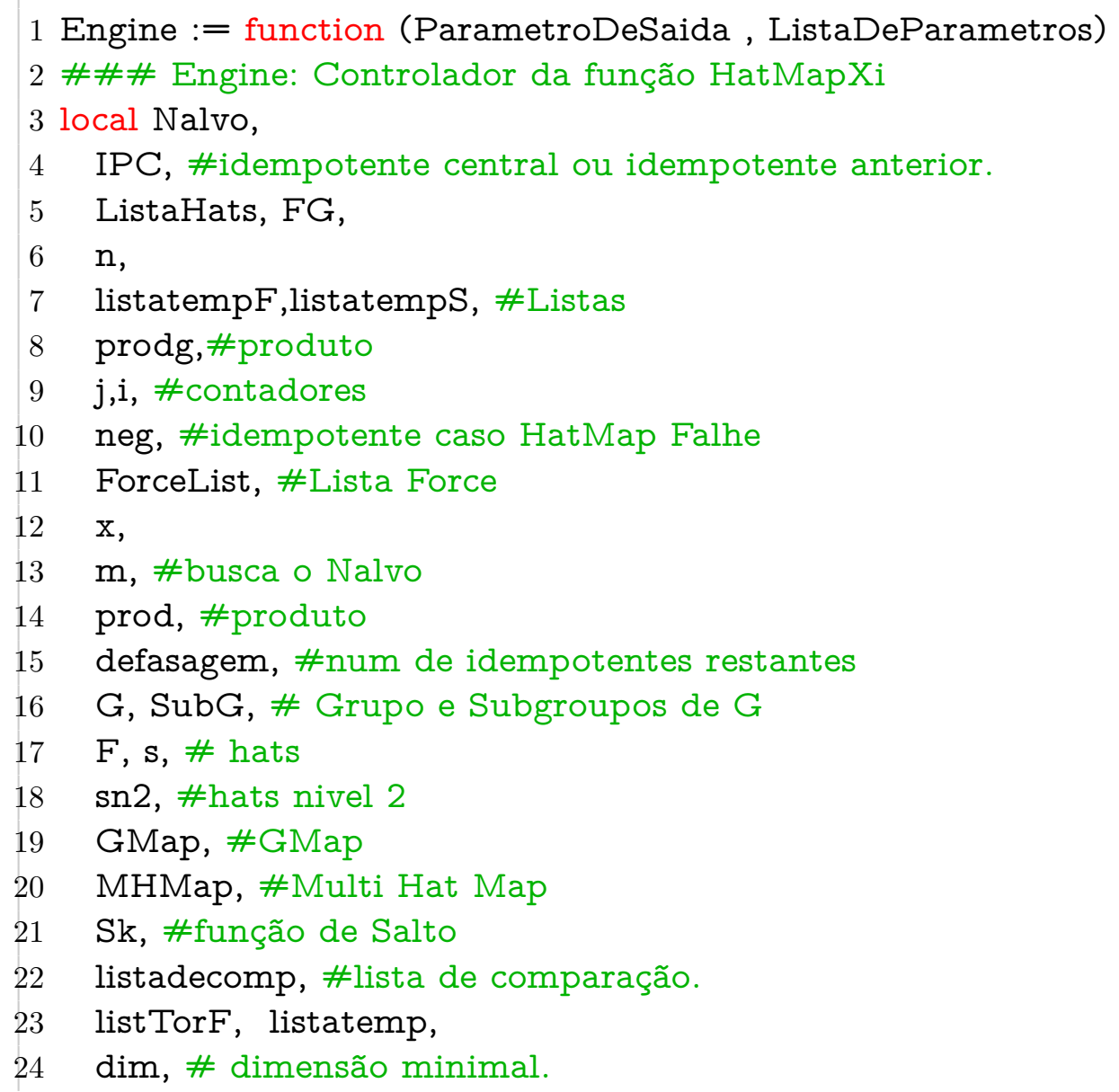


34 if ParametroDeSaida $=1$ then \# Núcleo de Engine

35 \# retorna para a função principal dados como:

$36 \quad \# \mathrm{k}$ em q=p $\mathrm{p}^{-\mathrm{k}}$

37 \# o elemento da próxima camada.

38

39 FG:= ListaDeParametros[4];

$40 \mathrm{G}:=$ List(UnderlyingMagma(ListaDeParametros[4])); \# G como lista

$41 \mathrm{~F}:=$ LeftActingDomain(ListaDeParametros[4]);

42 Nalvo:= ListaDeParametros[1];

43 IPC:= ListaDeParametros[2];

44

45 \#Entrada da dimensão.

46 if ListaDeParametros[5] $=0$ then

47 dim:=Dimension (Center(FG*IPC)); Encontra $\mathrm{k}$ em $\mathrm{p}^{\wedge} \mathrm{k}$

48 else

$49 \operatorname{dim}:=$ ListaDeParametros[5];

50 fi;

51

52 ListaHats:= ListaDeParametros[3];

53 SubG:= Reversed(Subgroups(UnderlyingMagma(ListaDeParametros[4])) ) ;

54

$55 \mathrm{n}:=$ Size (ListaHats);

$56 \mathrm{~m}:=0 ; \mathrm{x}:=0 ; \mathrm{i}:=1 ; \operatorname{dima}:=0$;

57 listatempF:=[]; \# lista temporária F

58 listatempS:=[]; \# lista temporária Somas linear combin.

59 listadecomp :=[Zero(FG)];

60

61 while $\mathrm{m}<$ Nalvo and $\mathrm{i}<=\mathrm{n}$ do

62 if $\operatorname{not}(\operatorname{prod}=\operatorname{Zero}(F G))$ then

63 dima:= ProgTestT (FG, prod ,SubG[i], dim); 
64 if dima $=\operatorname{dim}$ and IsIdempotent(prod) then \#deixar IdIdempotent em subnivel

$65 \mathrm{~m}:=\mathrm{m}+1 ;$ \#achou $\circ \mathrm{m}+1$-esimo.

$66 \quad \mathrm{x}:=\mathrm{i}$

67 else

$68 \mathrm{~m}:=\mathrm{m}+0 ;$ \#caso em que não seja dim 1

$69 \quad$ fi;

70 fi;

$71 \mathrm{i}:=\mathrm{i}+1$;

72 od;

73

74 \#\#\# Estudo da saída:

75 if $\mathrm{x} \ll>0$ then

76 return $[[[\mathrm{x}]], \operatorname{dim}]$;

77 else

78 return [[[]],dim];

79 fi;

80 \#\#\#

81 \#\#\#\# Fim do Parametro 1

82 \#\#\#\#\#\#

83

84 elif ParametroDeSaida $=2$ then \# Construtor e Reindexador de Hats

85 \#Organiza a progressão em termos do Lattice de $G$

$86 \quad F G:=$ ListaDeParametros[4];

87 G:= UnderlyingMagma(ListaDeParametros[4]);

$88 \quad F:=$ Left ActingDomain(ListaDeParametros[4]);

89

$90 \quad \mathrm{~s}:=\operatorname{Hats}(\operatorname{Subgroups}(G), F G) ;$

91 return s;

92 \#\#

93 \#\#\#\# Fim do Parâmetro 2

94 \#\#\#\#\#\#\#

95

96 elif ParametroDeSaida $=3$ then \#Interpretador de Posição

97 funcmap := function(n)

98 return $\mathrm{n}$;

99 end;

100 return funcmap;

101 \#\#

102 \#\#\#\# Fim do Parâmetro 3 


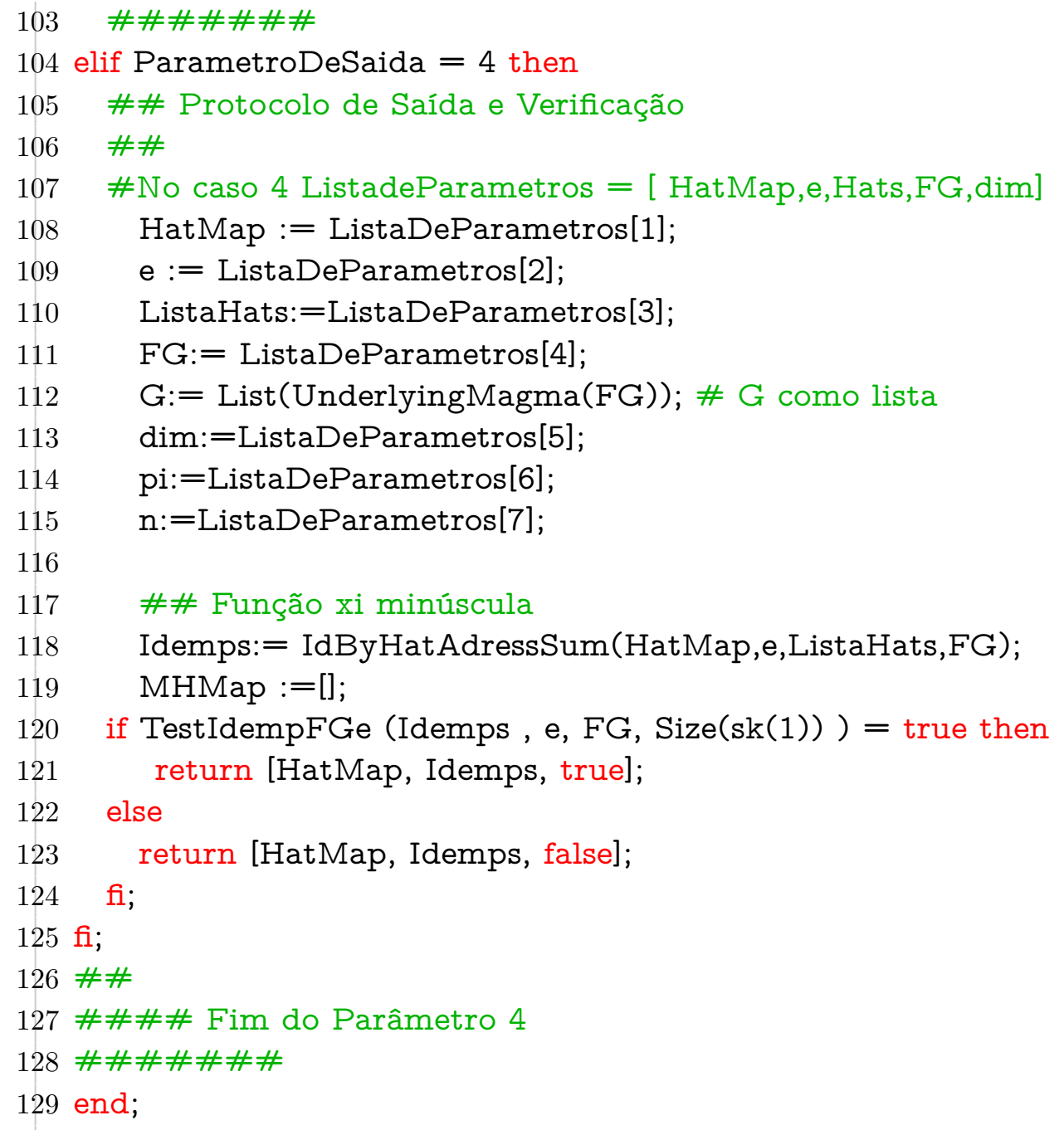

Dado um endereço $\mathscr{A}$, devemos conseguir recuperar seus idempotentes primitivos.

A função IdByHatAdressSum faz o trabalho das funções $\xi$ aplicadas em $\mathscr{A}$.

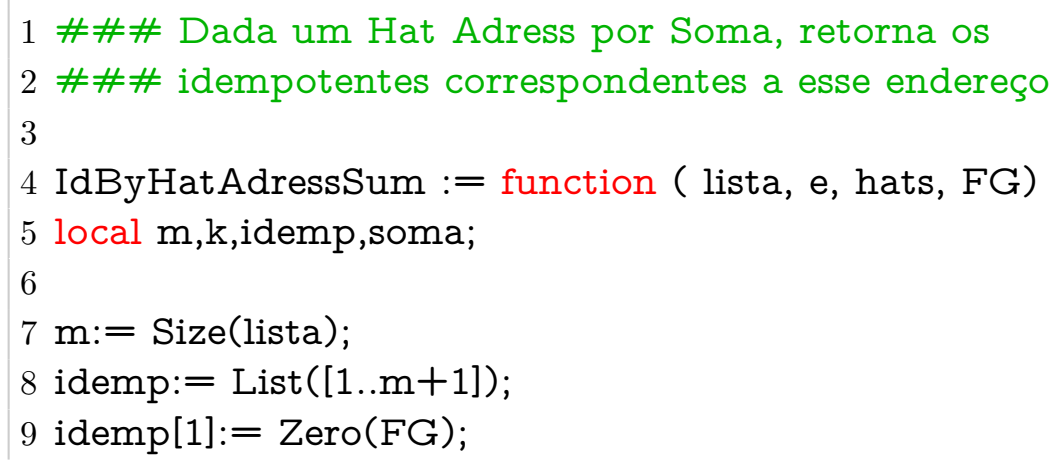


10 soma:=Zero(FG);

11 for $\mathrm{k}$ in $[2 . . \mathrm{m}+1]$ do

12 idemp $[\mathrm{k}]:=(\mathrm{One}(\mathrm{FG})$-soma)* hats [ lista[k-1] ]* (One(FG)-soma)*e ;

13 soma:= soma+idemp[k];

14 od;

15 Remove (idemp, 1);

16 return idemp;

17 end;

Dessa forma, retornando ao nosso exemplo anterior, em $\mathbb{F}_{2} G_{[27,4]}$, com o mesmo idempotente central e,se tomarmos $s_{k}(n)=(n, 1,1)$ então

$\operatorname{HatMapXi}\left(1, \mathbf{e}, F G, s_{k}\right)=[3,4,1]$

é o endereço da primeira família de $\mathscr{M}(\mathbf{e})$. No caso, é o endereço dos mesmos idempotentes obtidos no exemplo do ínicio do capítulo, obtido pelo Par de Shoda $\left(<a>, 1_{G}\right)$.

Já, se retomarmos ao exemplo em $\mathbb{F}_{2} G_{[27,4]}$, para o mesmo idempotente utilizado e, e para $s_{k}(n)=(n, 1,1)$ temos que

$$
\begin{aligned}
\operatorname{HatMapXi}\left(1, \mathbf{e}, F G, s_{k}\right) & =[3,4,1] \\
\operatorname{HatMapXi}\left(4, \mathbf{e}, F G, s_{k}\right) & =[6,7,1] \\
\operatorname{HatMapXi}\left(7, \mathbf{e}, F G, s_{k}\right) & =[9,10,1] \\
\operatorname{HatMapXi}\left(10, \mathbf{e}, F G, s_{k}\right) & =[12,13,1]
\end{aligned}
$$

São endereços de $\mathscr{M}(\mathbf{e})$ correspondentes a 4 famílias completamente distintas de idempotentes de $\mathbb{F}_{2} G_{[27,3]}$.

Como mencionado, o algoritmo descrito é extremamente versátil pois seu sucesso depende apenas da disposição do reticulado do grupo com relação ao idempotente central e. Vale ressaltar que há situações em que o HatMap como descrito é vazio, caso em que trataremos logo a seguir. Faremos no capítulo de exemplos uma extensa lista de exemplos e análise de situações. 


\subsection{Uma alternativa computacional}

Com o propósito de criar um algoritmo unificado, isto é, que não imponha hipóteses sobre $\mathbb{F} G$ senão sua finitude, precisamos lidar com casos em que $\Xi$, via HatMapXi, retorna um endereço incompleto, ou seja, quando $\mathbb{F} G \mathbf{e}=M_{n}\left(\mathbb{F}_{q}\right)$, mas $\mathscr{M}(\mathbf{e})$ não possui endereços de comprimento $n$. Uma enorme vantagem ao fazermos uso de um algoritmo unificado é podermos estudar os idempotentes de diferentes álgebras de grupo sobre o mesmo ponto de vista.

\subsubsection{Endereços Complementares}

Criaremos agora duas novas funções tais que combinadas, facilitarão o acesso aos idempotentes de $\mathbb{F} G \mathbf{e}$ por meio de outros tipos de endereços. Chamaremos de $\xi_{\mathfrak{h}}$ nossa função $\xi$ definida anteriormente. Já, as funções $\xi_{\mathfrak{m} \mathfrak{h}}$ e $\xi_{\mathfrak{f}}$ possuem o propósito de atender demais necessidades.

Caso o número de idempotentes obtido por $\xi_{\mathfrak{h}}$ não seja o esperado, ou seja, se $\mathbb{F} G \mathbf{e} \cong M_{n}\left(\mathbb{F}_{q}\right)$, mas o número de idempotentes ortogonais obtido por $\xi_{\mathfrak{h}}$ for inferior a $n$, prosseguiremos com a função de redução dimensional $\xi_{\mathfrak{m} \mathfrak{h}}$, pelo método que chamaremos de MultiHat e em seguida aplicaremos a função $\xi_{\mathfrak{f}}$ para de fato encontrar idempotentes pelo método que chamaremos de Force.

Para realizar o método, partiremos de um conjunto de $n_{0}$ idempotentes e encontraremos cada idempotente restante, um a um, pela sucessiva aplicação de $\xi_{\mathfrak{m} \mathfrak{h}}$ e $\xi_{\mathfrak{f}}$ respectivamente no conjunto inicial de idempotentes. Assim, uma única aplicação resultará em um conjunto com $n_{0}+1$ idempotentes. Reiniciaremos o processo até encontrar os $n$ idempotentes esperados.

Por fim, o produto final dessa abordagem será um endereço misto, ou seja, um $\left(\xi_{\mathfrak{h}},\left[\xi_{\mathfrak{m} \mathfrak{h}}, \xi_{\mathfrak{f}}\right]\right)-$ Adress, ou seja, um endereço que depende das 3 funções $\xi_{\mathfrak{h}}, \xi_{\mathfrak{m} \mathfrak{h}}$ e $\xi_{\mathfrak{f}}$ da forma:

$$
\mathscr{A}=\left[\mathscr{A}_{\mathfrak{h}},\left[\mathscr{A}_{\mathfrak{m} \mathfrak{h}}, \mathscr{A}_{\mathfrak{f}}\right]_{1}, . .,\left[\mathscr{A}_{\mathfrak{m} \mathfrak{h}}, \mathscr{A}_{\mathfrak{f}}\right]_{n-n_{0}}\right]
$$

Assim como na seção anterior, cada endereço será dependente de uma função de salto $s_{k}(n)$. Utilizaremos uma única função $s_{k}$ para todos os endereços visto que a utilização de diferentes funções para diferentes tipos de endereços é equivalente uma única função dada pela justaposição das m-úplas resultantes. 


\subsubsection{Método da Redução de Dimensão}

Seja $e$ um idempotente não primitivo qualquer de $\mathbb{F} G \mathbf{e} \cong M_{n}(\mathbb{F})$.

Queremos explorar a existência de elementos da forma

$$
\alpha=e . s_{i_{1}} s_{i_{2}} \ldots s_{i_{k}} e . \mathbf{e}
$$

tal que $\alpha$ seja um elemento não nulo de $e \mathbb{F} G e \mathbf{e}$ tal que gere um ideal de dimensão inferior que a de $e \mathbb{F} G e \mathbf{e}$.

Entretanto, tal tarefa pode ser demasiadamente custosa, visto que há $|\mathcal{S}|^{k}$ elementos candidatos a um possível $\alpha$. Por tal motivo as próximas construções visam criar um conjunto de resultados que serão descartados, reduzindo drasticamente a complexidade da tarefa. Devemos para tal, observar algumas situações.

1. O caso em que $s_{i_{1}}$ e $s_{i_{2}}$ possuem relação de subgrupo e,

2. O caso em que $s_{i_{1}}, s_{i_{2}}$ e $s_{i_{3}}$ são tais que $s_{i_{1}} s_{i_{3}}=0 \operatorname{com}\left[s_{i_{1}}, s_{i_{2}}\right]=0$

3. O caso em que $s_{i_{1}}$ e $s_{i_{2}}$ são tais que $s_{i_{1}} s_{i_{2}}=0$

Se $\alpha=e . s_{i_{1}} s_{i_{2}} \ldots s_{i_{k}}$ e.e possuir em seu produto elementos consecutivos como nos casos 1,2 e 3 , então $\alpha$ é nulo ou $\alpha$ é igual a um outro $\alpha^{\prime}$ com um número de produtos inferior em sua composição. Vamos buscar primeiramente elementos dessa forma formando assim nosso conjunto de descarte.

Considere $\mathfrak{a}_{E}(X)=\{s \in \mathcal{S} \mid s x=0, \forall x \in X\}$ o conjunto dos hats que se anulam à esquerda, $E_{E} t_{E}(X)=\{s \in \mathcal{S} \mid s x=x, \forall x \in X\}$, hats estáveis à esquerda e $\mathscr{C}_{\mathcal{S}}(X)=\{s \in \mathcal{S} \mid[s, X]=0\}$, os hats que comutam com todos os elementos de $X$. Utilizaremos os $\mathfrak{a}_{E}(X)$, Est $_{E}(X)$ e $\mathscr{C}_{\mathcal{S}}(X)$ para construir conjuntos de remoção à direita $C_{r_{i}}^{D}$ dados por

$$
\begin{aligned}
& C^{D} r_{0}=\{e\} \\
& C^{D} r_{1}=\mathfrak{a}_{E}(e) \cup E s t_{E}(e) \\
& C^{D} r_{2}=\left(\mathscr{C}_{\mathcal{S}}\left(C^{D} r_{1}\right) \cap \mathfrak{a}_{E}\left(C^{D} r_{0}\right)\right) \cup E s t_{E}\left(C^{D} r_{1}\right) \\
& \vdots \\
& C^{D} r_{i}=\bigcup_{k=1}^{k=i-1}\left(\left(\bigcap_{j=k}^{j=i-1} \mathscr{C}_{\mathcal{S}}\left(C^{D} r_{j}\right)\right) \cap \mathfrak{a}_{E}\left(C^{D} r_{k-1}\right)\right) \cup E s t_{E}\left(C^{D} r_{i-1}\right)
\end{aligned}
$$

Considere agora os conjuntos $\mathfrak{a}_{D}(X)=\{s \in \mathcal{S} \mid x s=0, \forall x \in X\}$ o conjunto dos hats que se anulam à direita de elementos de $X$, e o conjunto dos hats estáveis à direita, $E_{D} t_{D}(X)=\{s \in \mathcal{S} \mid x s=x, \forall x \in X\}$. De forma semelhante: 


$$
\begin{aligned}
& C^{E} r_{0}=\{e\} \\
& C^{E} r_{1}=\mathfrak{a}_{D}(e) \cup E s t_{D}(e) \\
& C^{E} r_{2}=\left(\mathscr{C}_{\mathcal{S}}\left(C^{E} r_{1}\right) \cap \mathfrak{a}_{D}\left(C^{E} r_{0}\right)\right) \cup E s t_{D}\left(C^{E} r_{1}\right) \\
& \vdots \\
& C^{E} r_{i}=\bigcup_{k=1}^{k=i-1}\left(\left(\bigcap_{j=k}^{j=i-1} \mathscr{C}_{\mathcal{S}}\left(C^{E} r_{j}\right)\right) \cap \mathfrak{a}_{D}\left(C^{E} r_{k-1}\right)\right) \cup E s t_{D}\left(C^{E} r_{i-1}\right)
\end{aligned}
$$

Os conjuntos $C^{D} r_{i}$ e $C^{E} r_{i}$ correspondem respectivamente ao conjunto dos hats que serão removidos quando vistos como componentes à esquerda de $e$ e à direita de $e$, ou então, às camadas de remoção à esquerda e à direita. Seja $R_{i}$ o conjunto de remoção dado por:

$$
R_{i}=\left(C^{E} r_{i} \cup C^{D} r_{n-i+1}\right), 0 \leq i \leq n
$$

Podemos, com cada $R_{i}$ definir:

Definição 2.13 Definimos a i-ésima camada multihat referente $e$ como sendo as camadas:

$$
C_{i}=\mathcal{S} \backslash R_{i}, 1 \leq i \leq n
$$

Além disso, definimos as camadas $C_{0}$ e $C_{n+1}$ como $C_{0}=C_{n+1}=\{e\}$

Dessa forma se $s_{i_{k}} \in R_{i}$ então, ou a $s_{i_{k}}$ retirada de $s_{i_{k}}$ do produto não altera $\alpha$, ou $s_{i_{k}}$ se anula em pelo menos uma das camadas adjacentes $\left(C_{i-1}\right.$ ou $\left.C_{i+1}\right)$, ou, no caso em que $x$ comuta com as uma ou mais camadas adjacentes sucessivamente, se anula em alguma delas.

As construções das camadas $C$ como apresentadas dão origem a um algoritmo consideravelmente mais econômico na busca por elementos redutores de dimensão $\alpha$.

O algoritmo ShellMaker é responsável por criar os conjuntos de remoção $C^{E} r_{i}$ e $C^{D} r_{i}$ e depende de uma segunda função ComutSL, cujo código fonte será deixado no apêndice.

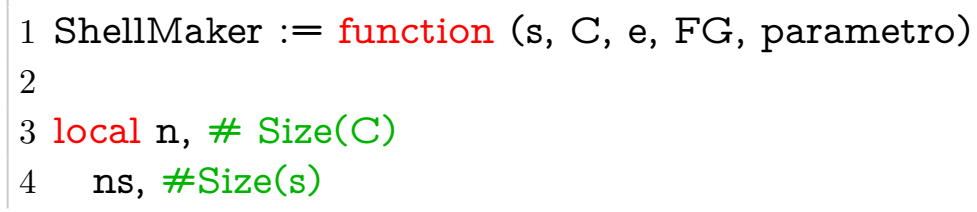


5 se, \#[s $1, \ldots, \mathrm{sn}, \mathrm{e}]$

6 i, \# contador

$7 \mathrm{j}$, \# contador

$8 \mathrm{k}$, \#contador

9 lista, \#lista anterior

10 list1, \#recebe ComutSL

11 list2, \#Intersection

$12 \mathrm{lE}$, \#lista esquerda

$13 \operatorname{lrE}$, \# lista a remover esquerda

$14 \operatorname{lrD}$,\#lista a remover direita

15 1D, \#lista direita

16 lr; \#lista de remoção lr $=[1 \mathrm{E}, 1 \mathrm{D}]$ Lista à direita e à esquerda

17

$18 \mathrm{n}:=\operatorname{Size}(\mathrm{C}) ; \mathrm{ns}:=\operatorname{Size}(\mathrm{s})$;

19

20 se := Flat ([s,e]);; \#adiciona o idempotente na ultima coordenada.

21

22 \#\#\# Captura os que são não nulos

23 \#\# Criação da camada trivial (idemp) e primeira camada

24

25 if $\mathrm{C}=[\mathrm{]}$ then

26 list $1:=[1 . . \mathrm{ns}]$;

27 if parametro $=-1$ then

$28 \mathrm{lD}:=$ Positions (List (list1, $\mathrm{x}->(\mathrm{s}[\mathrm{x}] * \mathrm{e}<>\operatorname{Zero}(\mathrm{FG})$ and $(\mathrm{s}[\mathrm{x}] * \mathrm{e}<>\mathrm{e})$ )), true);

$29 \operatorname{return}[$ Flat $([1,1 \mathrm{D}]),[\mathrm{ns}+1]]$;

30 else

$31 \mathrm{lE}:=$ Positions (List (list1, $\mathrm{x}->(\mathrm{e} * \mathrm{~s}[\mathrm{x}]<>\operatorname{Zero}(\mathrm{FG})$ and $(\mathrm{s}[\mathrm{x}] * \mathrm{e}<>\mathrm{e}))$

), true );

32 return $[[n s+1], F l a t([1,1 E])]$;

33 fi;

34 fi;

$35 \mathrm{lD}:=[] ; \mathrm{lE}:=[] ; \operatorname{lrD}:=[] ; \operatorname{lr} \mathrm{E}:=[] ;$

36 lista:=List $([1 . . \mathrm{ns}])$;

37

38 \#\#\#

39 \#\# Demais camadas

40 \#

41 if parametro $=1$ then

42 for $\mathrm{j}$ in [2..n] do 
43

$77 \mathrm{lE}:=$ List $([1 \ldots \mathrm{ns}]) ;$;

78 lD:=List ([1..ns]);

79 SubtractSet (lE, Flat(lrE)); \#Remoção de conjuntos

80 SubtractSet (ID, Flat(lrD)); \#Remoção de conjuntos 
81

82 if parametro $=1$ then

83 return $\mathrm{lE}$;

84 else

85 return $1 D$;

86 fi;

87

88 end;

Estamos entretanto interessados em idempotentes de $\mathbb{F} G \mathbf{e}$ ortogonais ao idempotente $e_{\text {sum }}=\left(e_{1}+\ldots+e_{n_{o}}\right) \in \mathbb{F} G \mathbf{e}$. Assim, vamos encontrar um elemento redutor de dimensão utilizando o idempotente

$$
e_{\text {neg }}=\left(1-e_{\text {sum }}\right) \mathbf{e}=\left(1-\left(e_{1}+\ldots+e_{n_{o}}\right)\right) \mathbf{e}
$$

Neste caso, diremos que um elemento da forma

$$
\begin{aligned}
\alpha & =\left(1-e_{\text {sum }}\right) \cdot s_{C_{1}} \cdot s_{C_{2}} \ldots s_{C_{k}}\left(1-e_{\text {sum }}\right) \cdot \mathbf{e} \\
& =e_{n e g} s_{C_{1}} \cdot s_{C_{2}} \ldots s_{C_{k}} e_{n e g} \cdot \mathbf{e}
\end{aligned}
$$

possui endereço MultiHat $\left[i_{1}, i_{2}, \ldots, i_{k}\right]_{\mathfrak{m} \mathfrak{h}}$, em que $s_{C_{i_{k}}} \in S \cap C_{i}$, ou seja, o conjunto de elementos de $S$ que não foram removidos.

Suponhamos que ao procurar por endereços em $\mathscr{M}(\mathbf{e})$ obtenhamos um endereço incompleto $\mathscr{A}$ de comprimento $n_{o}$. Dessa forma, $e_{i}=\xi_{\mathfrak{h}}\left(\left.\mathscr{A}\right|_{i}\right)$, com $1 \leq i \leq n_{o}$ são os primeiros $n_{o}$ idempotentes primitivos de $\mathbb{F} G$ e. No caso em que $\mathscr{A}=\emptyset$, então $n_{o}=0$.

1 MultiHatsSS := function ( $F G$, e,dimmin, iterac, $m$ )

2 local $\mathrm{n}$, \#tamanho inicial dos hats

3 nit, \# número da iteracao

4 s, \# Hats básicos

5 sa, \# lista a

$6 \mathrm{sb}$, \# lista $\mathrm{b}$

7 G,

8 i, \# contador

$9 \mathrm{j}$, \# contador

10 lasti, \# i referente ao último idempotente encontrado

11 mo, \#m-ésimo idempotente

$12 \mathrm{y}$, \# elemento de FG candidato a idempotente

13 id, \#idempotente encontrado

14 adr, \#endereço 
15 adr2, \#último endereço válido encontrado

16 adrClean, \# endereço final sem 1s

17 nzhatsindex, \# não-zero Hats index

18 nzhsize, \# tamanho de nzhatsindex

19 Preadr, \#pre-adress

20 rPreadr, \# pre-adress reverso

21 dimy, \#dim y

22 miniindex, \# i correspondente a y com dim mínima encontrada

23 miniindexSet, \# conjunto de i com dimensões mínimas na ordem inversa

24 dimpack, \# coletor de dimensões

25 SM, \# recebe [1E, 1D] de ShellMaker

26 dimpacklast, \# armazena dimpack

27 nextcomp, \# comparacao das dimensões

28 mbaseorders,

29 C,

30 CompDireita,

31 CompEsquerda,

32 indexcomp, \#nivel da proxima comparacao

33 nmax; \#n da iteração máxima

34

$35 \mathrm{G}:=$ UnderlyingMagma(FG);

$36 \mathrm{~s}:=\operatorname{Hats}(\operatorname{Subgroups}(G), F G) ;$

$37 \mathrm{n}:=\operatorname{Size}(\mathrm{s})$;

38 nzhatsindex := Positions(List(s, $\mathrm{x}->\mathrm{x}<>\operatorname{Zero}(\mathrm{FG}))$, true);

39

40 \#Preparação de Parâmetros

41 nit:=1; sa:=s; sb:=[]; $:=0 ;$ lasti $:=0 ; \mathrm{mo}:=0$;

42 adr:=[];adr2:=[1];id:=Zero(FG);dimpack:=[];dimpacklast:= [];

43

44 \#\#\#\# Análise de Camadas

45 \#\#\#\# Obtendo Camadas:

46 \# Camadas tem tamanho iterac +2

47 \# camadas são da forma e.s1'.s2'.......s2 . s1. e

48 \# em forma de camadas: $\mathrm{C}=\mathrm{c} 1^{\prime} . c 2^{\prime}$ c3'....... c3 c2 .c1

49 \# c'1 e c1 são referentes ao idempotente, serão marcadas com 0.

50

$51 \mathrm{C}:=$ [] ; \#constrói camada 1 (trivial) e 2

52 CompEsquerda := [];CompDireita $:=[]$;

53 
54 \#Primeira e segunda Camada:

55

56 Add(CompEsquerda, ShellMaker (s, C, e, FG,1));

57 Add(CompDireita, ShellMaker (s, C, e, FG,-1), 1);

58 CompDireita:=CompDireita[1];

59 CompEsquerda:=CompEsquerda[1];

60

61 for $\mathrm{i}$ in [2.. iterac] do

62 Add(CompEsquerda, ShellMaker (s, CompEsquerda, e, FG,1));

63 Add(CompDireita, ShellMaker (s, CompDireita, e, FG,-1), 1); \#\# Insere na ordem inversa

64 od;

65

66 \# Retirando o índice do idempotente:

67 Remove (CompEsquerda, 1);

68 Remove (CompDireita, Size(CompDireita));

69

70 \# fazendo a sobreposição de camadas

71 for $i$ in [1..iterac] do

72 if Intersection ( CompEsquerda [i] , CompDireita[i] ) $<>$ [] then

73 Add ( C, Intersection ( CompEsquerda [i] , CompDireita[i] ));

74 else

75 Add ( C, [1] );

76 fi;

77 od;

78

79 \# Operando com o conjunto Reindexado

80 nzhsize:= Size(nzhatsindex);

81 mbaseorders:= List (C, $\mathrm{x}->\operatorname{Size}(\mathrm{x})$ );

$82 \mathrm{nmax}:=$ Product (mbaseorders);

83

84 miniindex $:=[\mathrm{nmax}]$

85

86 \#\#\#\# Nextcomp ATIVADO

87 indexcomp:=Size(mbaseorders);

88 nextcomp :=mbaseorders [indexcomp] ;

89

90 \# primeira comparação depois que acaba a segunda iteração

91

92 dimpack :=[dimmin*iterac^2]; \#Dimensão Máxima 
93

$94 \mathrm{i}:=1$; \#\# i começa em 1 ignorando o produto $[1,1 . ., 1]$;

95 while $\mathrm{i}<=$ nmax and mo $<\mathrm{m}$ do

96 Preadr:= Vetorize2multibase(i,mbaseorders);

97 adr:= List ([1..iterac], $\mathrm{x}->\mathrm{C}[\mathrm{x}][$ Preadr $[\mathrm{x}]])$;

98 Print ("\n Pre-Endereco: ", Preadr, " Enderecp: ", adr );

$99 \mathrm{y}:=$ e*Product ( $\operatorname{adr}, \mathrm{x}->\mathrm{s}[\mathrm{x}]$ )*e;

100 if $\mathrm{y}<>\operatorname{Zero}(\mathrm{FG})$ then

101 dimy:= ProgTest(FG, y,dimmin); \# Armazena sua Dimensão

102 if dimy $<=$ Minimum (dimpack) then \#\#\#

103 Add (dimpack, dimy, 1 ); \#armazena lista na ordem inversa

104 Add (miniindex, i, 1);

105 else

106 Add (dimpack, dimy); \#joga a dimensão pro final da lista

107 fi;

108 if dimy $=$ dimmin then

109 mo:= mo+1; \#encontrado o m-ésimo idempotente (função de salto)

$110 \quad$ lasti: $=\mathrm{i}$;

$111 \quad \mathrm{id}:=\mathrm{y} ;$ \#último idempotente encontrado

$112 \quad \operatorname{adr} 2:=\operatorname{adr}$;

113 fi;

114 fi;

115 if $\mathrm{i}=$ nextcomp then \#verificação de estabilização de dimensão

116 if Set(dimpack) = Set(dimpacklast) then \#dimensões estabilizaram

$117 \quad \mathrm{i}:=\mathrm{nmax}+1$; \#sai do loop

118 else

119 dimpacklast:= dimpack;

120 dimpack:=[iterac ${ }^{` 2}$ ];

121 if indexcomp-1 $>0$ then

122 nextcomp:=nextcomp $*$ mbaseorders[indexcomp-1];

123 else

124 nextcomp:=nmax;

125 fi;

126 fi;

127 fi;

$128 \mathrm{i}:=\mathrm{i}+1$;

129 od;

130

131 if $\mathrm{mo}=0$ then 
132 \# retornará o m-ésimo idempotente de menor dimensão

133 \# após estabilização

134

135 miniindexSet $:=$ Positions (dimpack, Minimum (dimpack)) ;

136

$137 \mathrm{i}:=$ miniindex $[$ Size (miniindexSet) $+1-\mathrm{m}]$;

138

139 Preadr:= Vetorize2multibase(i,mbaseorders);

$140 \operatorname{adr} 2:=$ List ([1..iterac], $\mathrm{x}->\mathrm{C}[\mathrm{x}][$ Preadr $[\mathrm{x}]])$;

141

142 adrClean :=[];

143 for $i$ in [1..Size(adr2)] do \#\# Retirar "1"s:

144 if $\operatorname{adr}[\mathrm{i}]<>1$ then

145 Add (adrClean, adr[i]);

146 fi;

147 od;

148

$149 \mathrm{y}:=$ e*Product ( $\operatorname{adr} 2, \mathrm{x}->\mathrm{s}[\mathrm{x}]) * \mathrm{e}$;

150

151 return [y, adrClean,0] ;

152 else

153 adrClean :=[];

154 for $\mathrm{i}$ in [1..Size(adr2)] do \#\# Retirar "1"s:

155 if $\operatorname{adr} 2[\mathrm{i}] \ll>1$ then

156 Add (adrClean, adr[i]);

157 fi;

158 od;

159

160 \#\# devolve 1 caso todos sejam removidos

161 \#\# com um idempotente valido.

162 if adrClean $=[]$ then

$163 \quad$ adrClean :=[1];

164 fi;

165 return [y, adrClean,1] ;

$166 \mathrm{fi} ;$

167 end;

Notemos que o elemento $\alpha$ não necessariamente é um idempotente, mas $e_{n e g} \cdot e_{\text {sum }}=$ $e_{\text {sum }} e_{\text {neg }}=0 \log \mathrm{O}$,

$$
\alpha e_{i}=e_{i} \alpha=0, \forall 1 \leq i \leq n_{o}
$$


Além disso, $\alpha \in \mathbb{F} G \mathbf{e}$ e o ideal $\mathbb{F} G \alpha \mathbf{e}=\mathbb{F} G \alpha$ contém pelo menos um idempotente primitivo ortogonal aos idempotentes $e_{1}, \ldots, e_{n_{o}}$.

Calculado o elemento $\alpha$ por meio de MultiHat precisamos agora da segunda parte do endereço para que, de fato, encontremos idempotentes.

Sabemos que $\{g\}_{g \in G}$ determina uma $\mathbb{F}$-base para $\mathbb{F} G$ bem como gera qualquer subespaço de $\mathbb{F} G$. Consideremos então a $\mathbb{F}$-base $\beta_{1}=\left\{g_{1}, . ., g_{n}\right\}_{g_{i} \in G}$, ou seja, o mesmo conjunto $\{g\}_{g \in G}$, fixada uma ordem para os elementos de $G$. Uma vez dado o idempotente $e_{n e g}$ e $\alpha$, vamos estudar os idempotentes do ideal $I=\alpha \mathbb{F} G \alpha$. Suponha $\operatorname{dim}_{\mathbb{F}} I=k$

É evidente que o conjunto dos elemento $\beta_{2}=\left\{b_{i}=\alpha g_{i} \alpha \mathbf{e}\right\}_{g_{i} \in G}$ é um gerador de $M$ e que $k=\operatorname{dim}_{\mathbb{F}} M \leq \operatorname{dim}_{\mathbb{F}} \mathbb{F} G \mathbf{e}<|G|$. Portanto, será necessário reduzir $\beta_{2}$ a uma base de $I$. O faremos de maneira a minimizar os índices $i$ dos elementos $g_{i}$ de $G$, assim definimos $\beta_{\mathfrak{f}}$ como

$$
\beta_{\mathfrak{f}}=\left\{b_{i_{1}}, \ldots, b_{i_{k}}\right\} \subset \beta_{2}
$$

onde os índices $i_{1}, \ldots, i_{k}$ são tais que $i_{1}=I d_{G}$, e para todo índice $i_{x}, i_{x}$ é o menor índice tal que $\left\{b_{i_{1}}, \ldots, b_{i_{x}}\right\}$ seja L.I. sobre $\mathbb{F}$. O próximo algoritmo retorna duas listas, a primeira a qual chamaremos de base suja são os próprios elementos de $\beta_{\mathfrak{f}}$ e a segunda lista a qual chamaremos de base limpa corresponde aos elementos $g_{i}$ do grupo $G$ tais que $\alpha g_{i} \alpha \in \beta_{\mathfrak{f}}$. É importante destacar que os elementos de base limpa não formam uma base.

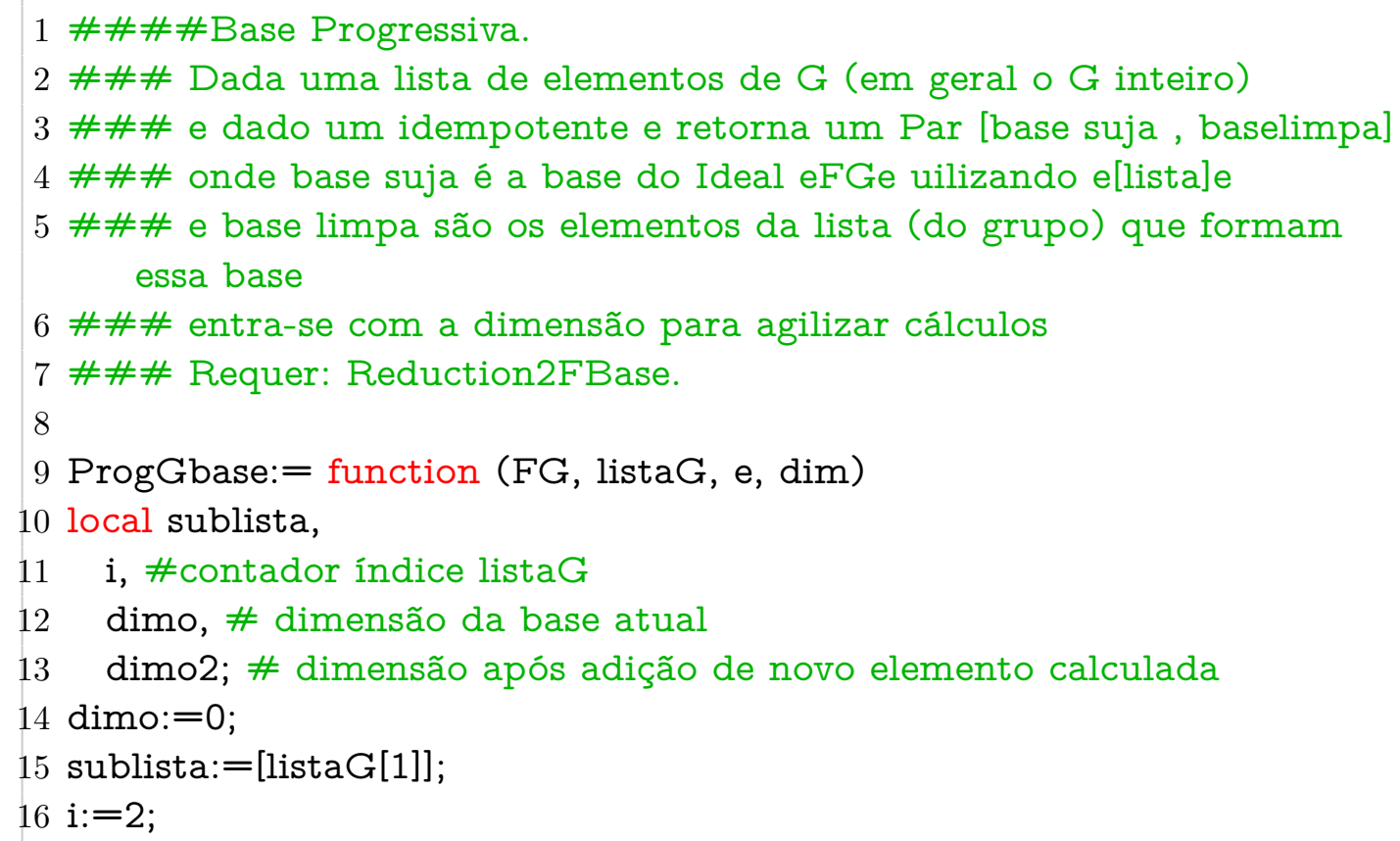


17 while dimo < dim do

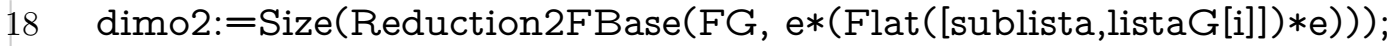

19 if dimo2 > dimo then

20 Add(sublista, listaG[i]);

21 fi;

22 dimo:=dimo2;

$23 \mathrm{i}:=\mathrm{i}+1$;

24 od;

25 return [e*sublista*e,sublista]; \#base suja e base limpa

26 end;

Consideremos agora os elementos de $\mathbb{F}=\left\{\lambda_{1}, . ., \lambda_{s}\right\}$ fixando-se também uma ordem em $\mathbb{F}$.

Agora os elementos de $I$ estão em correspondência com elementos da forma $\left[\alpha_{1}, . . \alpha_{k}\right]$ em $I_{1} \times I_{2} \times \ldots \times I_{k}$, onde $I_{1}=. .=I_{k}=\{1 . . s\}$ através de

$$
\left[\alpha_{1}, \alpha_{2}, . ., \alpha_{k}\right] \stackrel{f_{1}}{\longleftrightarrow} \lambda_{\alpha_{1}} b_{i_{1}}+. .+\lambda_{\alpha_{k}} b_{i_{k}}
$$

Estabelecida agora uma ordem em $I$ (lexicográfica induzida por $I_{1} \times I_{2} \times \ldots \times I_{k}$ ) diremos que dois elementos $x$ e $y$ de $\mathbb{F} G$ em $M$ são tais que $x<y$ quando seus respectivos correspondentes em $I_{1} \times I_{2} \times \ldots \times I_{k}$ satisfizerem a mesma relação.

Por fim, sabemos que em $M$ contabilizamos $s^{k}$ elementos e portanto $<$ induz uma enumeração dos elementos de $I$, ou seja, uma bijeção $f$ entre o intervalo de números inteiros $I_{\text {num }}=\left\{1,2, . ., s^{k}\right\}$ e $I$

$$
I_{\text {num }} \stackrel{f}{\longleftrightarrow} I
$$

Portanto, os idempotentes de $I$, bem como todo o ideal está em $\operatorname{Imf}=I$.

Os $\xi_{\mathfrak{f}}$-endereços para esta função serão da forma $\mathscr{A}=\left[A_{F}, A_{G}\right]$ onde $A_{F}$ e $A_{G}$ são úplas de mesmo comprimento dadas da seguinte maneira: se

$$
e=\sum_{\substack{\lambda_{x} \in \mathbb{F} \backslash\{0\} \\ b_{i_{x}} \in \beta}} \lambda_{x} b_{i_{x}}
$$

então $A_{F}=\left[. ., \alpha_{\lambda_{i}}, ..\right]$ e $A_{G}=\left[\ldots, i_{x}, ..\right]$. Ou seja, $A_{F}$ corresponde a $f^{-1}(e)=$ $\left(\alpha_{1}, \alpha_{2}, . ., \alpha_{k}\right)$, eliminando-se os coeficientes correspondentes aos elementos nulos de $\mathbb{F}$,já $A_{G}$, corresponde ao índice do suporte de $e$ em $\beta_{2}$. 
Sejam $\left(e_{1}^{I}, e_{2}^{I}, \ldots, e_{l}^{I}\right)$ os idempotentes de $I$ tais que

$$
e_{1}^{I}<e_{2}^{I}<\ldots<e_{l}^{I}
$$

e seja $m \in \mathbb{N}$.

Diremos que $\xi_{\alpha}^{\mathfrak{f}}(m)=\mathscr{A}_{e_{m}}$ se $1 \leq m<l$ e $\xi_{e}^{\mathfrak{f}}(m)=\mathscr{A}_{e_{l}}$ caso $m \geq l$.

O algoritmo ForceMidp é a implementação direta do exposto e encontra o endereço do $m$-ésimo idempotente do tipo $\mathfrak{f}$ para $\alpha$.

$1 \# \# \# \# \# \# \# \# \# \# \# \# \# \# \# \# \# \#$

2 \#\#\#\#\#\# Force m-ésimo idempodente

3 \#\# Retorna o idempotente e sua escrita em base limpa

respectivamente

4 \#\# Retorna: [idemp ,idemp limpo ,coord,baselimpa, G-Map];

5 \#\# onde G-Map é uma lista do tipo [ [F1, G1], [F2, G2] ...]

6 \#\# dim é a dimensão do submódulo

7 \#\# dimmin é a dimensão minimal referente ao idempotente sobre F. 8

9 ForceMidp:= function (FG, listaG, eforce, dim, $\mathrm{m}$, dimmin) 10

11 local mo, \#contador de idempotentes

12 n, \#tamanho do submódulo

13 basesuja,

14 baselimpa,

15 yforce, \# elemento do submódulo

16 ycforce, \# y clean

17 l, \#lista com xc e coord

18 coord,

19 posits,

20 positsn1, \#posições nao 1 em F. (elimina os Zeros)

21 GMap,\# G Mapa Limpo, ie sem coordenadas nulas

22 dimh, \#julgamento da dimensão pelo DimHate

23 iforce; \# contador

24

$25 \mathrm{mo}:=0$;

26

27 iforce: $=0$;

28 yforce:=false;

29 ycforce:=false;

30 
31 basesuja:= ProgGbase (FG, listaG, eforce, dim)[1];

32 baselimpa:= ProgGbase (FG, listaG, eforce, dim)[2];

$33 \mathrm{n}:=\operatorname{Size}($ LeftActingDomain(FG))`Size(basesuja);

34

35 while $\mathrm{mo}<\mathrm{m}$ and iforce $<=\mathrm{n}$ do $\# \mathrm{n}$ pois a unidade foi descartada e será reposta no final.

36 iforce:=iforce+1;

37 yforce:= Vetorize2Fbase (iforce, FG,basesuja)[1];

38 BPrint (" $\backslash \mathrm{n}$ Calculando yforce com iforce:=", iforce, " de ", n);

39 if (yforce ${ }^{\wedge}=$ yforce) and (yforce $<>$ Zero(FG)) then

40 \#\#\# Julgar a dimensão.

41 Print ("\n Analisando iforce:=", iforce, " de ", n);

42 dimh:= DimHate (FG, yforce, [One(FG)])[1];

43 Print ("\n Dimensao dimh:=", dimh);

44 if dimh $=$ dimmin then

$45 \mathrm{mo}:=\mathrm{mo}+1$

$46 \quad$ fi;

$47 \quad$ fi;

48 od;

49

50 if $\mathrm{mo}=\mathrm{m}$ then

$51 \mathrm{l}$ :=Vetorize2Fbase (iforce, FG, baselimpa);

52 ycforce: $=1[1]$;

53 coord: $=1[2]$;

54 fi;

55 posits:= List(baselimpa, $\mathrm{x}->$ Position (lista $G, \mathrm{x})$ );

56

57 positsn $1:=$ Positions (List (coord, $\mathrm{x}->\mathrm{x}<>1$ ), true);

58 GMap:= List (positsn1, $\mathrm{x}->$ [ $\operatorname{coord}[\mathrm{x}], \operatorname{posits}[\mathrm{x}]$ ] );

59

60

61 return [yforce,ycforce,coord,baselimpa, GMap];

62 end; 


\subsection{Um Conjunto Completo de Idempotentes Pri- mitivos em FG}

Consideradas todas as construções feitas na seção anterior, vamos finalmente descrever em termos dos endereços $\xi_{*}$ um conjunto completo de idempotentes primitivos de $\mathbb{F} G \mathbf{e}$. O algoritmo completo, apenas amarra os algoritmos induzidos pelas funções anteriores. A função $\xi_{\mathfrak{f}, \mathfrak{m} \mathfrak{h}, \mathfrak{h}}^{s_{k}, \mathbf{e}}=\xi_{\mathfrak{f}}^{s_{k}} \circ \xi_{\mathfrak{m} \mathfrak{h}}^{s_{k}, \mathbf{e}} \circ \xi_{\mathfrak{h}}^{s_{k}, \mathbf{e}}$, dada pela aplicação sucessiva das funções $\xi_{\mathfrak{h}}, \xi_{\mathfrak{m} \mathfrak{h}}$ e $\xi_{\mathfrak{f}}$ associa a cada $m \in \mathbb{N}$ um endereço composto da forma $\mathscr{A}=\left[\mathscr{A}_{\mathfrak{h}}, \mathscr{A}_{\mathfrak{m i s t o}-1}, \ldots \mathscr{A}_{\text {misto-k }}\right]$, ou seja, um endereço $\mathfrak{h}$ do tipo hat de comprimento $n_{o}$ referente aos $n_{o}$ primeiros idempotentes de $\mathbb{F} G \mathbf{e}$ e mais $k=n-n_{o}$ idempotentes do tipo $\mathscr{A}_{\text {misto }}=\left[A_{\mathfrak{m} \mathfrak{h}}, A_{\mathfrak{f}}\right]$ (necessariamente nessa ordem). No caso em que $\mathscr{A}_{\mathfrak{h}}$ possuir comprimento $n$, ou seja, se $k=0$ então não haverão endereços mistos, nesse caso, diremos que $\mathscr{A}=\mathscr{A}_{\mathfrak{h}}$ é do tipo $\mathfrak{h}$, ou seja hat - puro, caso contrário será um endereço composto.

Os endereços do tipo $\mathfrak{h}$ mostram-se extremamente relacionados com a disposição dos subgrupos no reticulado de $G$, como por exemplo no caso de $\mathbb{F}_{2} G_{[27,3]}$ e $\mathbb{F}_{2} G_{[27,4]}$, onde uma diferença na quantidade de subgrupos e na posição propícia dos subgrupos de $G_{[27,3]}$ permite um maior número de idempotentes distintos descritos por hats. As álgebras de grupo $\mathbb{F} G$ cujas componentes são todas descritas por endereços $\mathfrak{h}$ mostram-se abundantes e sua determinação permite estudar tais idempotentes sobre outro ponto de vista. Grupos com um demasiado número subgrupos normais, e.g. Hamiltonianos, podem levar à necessidade de prosseguir para os endereços mistos, tais endereços podem demandar um maior recurso computacional. Por outro lado, com pequenas adaptações é possível utilizar a HatMapXi de forma que a fazer uma leitura e reconhecimento de endereços de famílias completas Idempotentes Centrais Primitivos por meio de endereços $\mathfrak{h}$. Caso em que pode ser demasiadamente interessante. Tal abordagem será discutida posteriormente.

Corolário 2.14 Seja e um idempotente primitivo de $\mathbb{F} G$ tal que $\mathbb{F} G \mathbf{e} \cong M_{n}\left(\mathbb{F}_{p^{k}}\right)$ e seja $S=\{\widehat{H}\}_{H \leq G}$ com função de indexação $s(i)=s_{i} \in S$ e $s_{k}$ a função de salto. Seja $m$ tal que $\xi_{\mathbf{e}}^{s_{k}}(m)=\left[\mathscr{A}_{\mathfrak{h}}\right]=\left[i_{1}, i_{2}, . ., i_{n}\right]$. Então os idempotentes primitivos de $e_{1}, . ., e_{n}$ associados a $m$ são da forma

$$
\begin{aligned}
e_{j} & =\sum_{r=1}^{j}\left((-1)^{r+1} \sum_{j \geq k_{1} \geq \ldots \geq k_{r}} s_{i_{j}} \prod_{l=1}^{r} s_{k_{l}}\right) \mathbf{e}, j=1 . . n \\
& =s_{i_{j}} \Pi_{l}^{j-1}\left(1-e_{l}\right) \mathbf{e}
\end{aligned}
$$

As construções dependem basicamente de três informações previamente calculadas, em primeiro lugar, depende de um idempotente primitivo central e, além 
disso, depende implicitamente da dimensão da componente simples associada bem como sua dimensão de seu centro sobre $\mathbb{F}$, ou seja, depende de $n$ e $k$ tais que $\mathbb{F} G \mathbf{e} \cong M_{n}\left(\mathbb{F}_{p^{k}}\right)$. Assim, para iniciarmos o processo, a terna $(\mathbf{e}, n, k)$ deve ser previamente estipulada, mesmo antes de escolhermos uma função de salto $s_{k}$. Caso tais números não sejam fornecidos, eles são calculados automaticamente pelo Engine principal. Caso não seja escolhida uma função de salto, o Engine utiliza a função de salto padrão, isto é $s_{k}(n)=(n, 1, . ., 1)$ cujo comprimento é o mesmo que o número de idempotentes esperados.

Como mencionado no capítulo anterior, optamos majoritariamente pelo método dos Pares de Shoda visto que dado um par forte de Shoda $(H, K)$ de $G$, a terna $(\mathbf{e}, n, k)$ é imediatamente obtida facilitando a entrada e obtendo um ganho de tempo considerável para o algoritmo.

Entretanto, nosso método não é restrito a grupos fortemente monomiais nem mesmo à semissimplicidade de $\mathbb{F} G$, visto que os idempotentes centrais primitivos podem alternativamente ser obtidos pelo método da tabela de caracteres via PrimitiveCentralIdempotentsByCharacterTable presente no pacote $W$ edderga [4] , ao custo computacional extra para a determinação dos números $n$ e $k$. Já, para o caso não semissimples, pode-se determinar os idempotentes centrais primitivos pelo método da função de competição como será descrita adiante.

Outra vantagem que será abordada é a própria descrição de $\varepsilon$ por $(H, K)$, ambos subgrupos de $G$. Para o caso em que se opte por $S$ como o conjunto dos subgrupos de $G$, a função de indexação $s_{i}$ também pode ser utilizada para atribuir ao par $(H, K)$ seu respectivo endereço, ou seja $(H, K)=\left(s_{i_{H}}, s_{i_{K}}\right)$.

Exemplo 2.15 Consideremos o exemplo de $G_{[32,50]} \cong\left(C_{2} \times Q_{8}\right) \rtimes C_{2}$ em $\mathbb{F}_{3}$ temos que

$$
\mathbb{F}_{3} G \cong 16 \mathbb{F}_{3} \oplus M_{4}\left(\mathbb{F}_{3}\right)
$$

neste caso, para $e=\left(1-\widehat{G^{\prime}}\right)$ temos HatMapXi o seguinte endereço:

$$
\mathscr{A}=[[[3],[[3,1],[2,5],[2,11]]],[[3],[[2,1]]],[[1],[[3,1],[2,5],[2,11]]], 1]
$$

Ou seja, neste caso, não há endereços do tipo hy, mas todos do tipo misto com exceção do último endereço, sendo

$$
\mathscr{A}_{\mathfrak{m i s t o}-1}=[[3],[[3,1],[2,5],[2,11]]]
$$




$$
\begin{gathered}
\mathscr{A}_{\text {misto-2 }}=[[3],[[2,1]]] \\
\mathscr{A}_{\text {misto-3 }}=[[1],[[3,1],[2,5],[2,11]]] \\
\mathscr{A}_{h}=[1]
\end{gathered}
$$

Ou seja, o primeiro idempotente é calculado por:

$$
e_{1}=\xi\left(\mathscr{A}_{\mathfrak{m i s t o}-1}\right)=\widehat{H}_{3}\left(2 g_{1}+g_{5}+g_{11}\right) \widehat{H}_{3} \mathbf{e}
$$

pois possui coordenada multihat $A_{\mathfrak{m} \mathfrak{h}}=[3]$ e $A_{\mathfrak{f}}=[[3,1],[2,5],[2,11]]$, onde os elementos de $\mathbb{F}_{3}$ estão em bijeção com os elementos de $[1,2,3]$, ou seja, o número 1 corresponde ao elemento $0_{\mathbb{F}} \in \mathbb{F}, 2$ corresponde a $1_{\mathbb{F}} \in \mathbb{F}$ e 3 a $2_{\mathbb{F}} \in \mathbb{F}$. Tal indexação também nos permite trabalhar com corpos do tipo $\mathbb{F}_{p^{k}}$. Para $\mathscr{A}_{m i s t o-2}$, temos

$$
\begin{aligned}
e_{2}=\xi\left(\mathscr{A}_{\text {misto-2 }}\right) & =\left(1-e_{1}\right) \widehat{H}_{3}\left(g_{1}\right) \widehat{H}_{3}\left(1-e_{1}\right) \mathbf{e} \\
& =\left(1-e_{1}\right) \widehat{H}_{3}\left(1-e_{1}\right) \mathbf{e}
\end{aligned}
$$

e, como $g_{5}, g_{11} \in \mathscr{C}_{G}\left(e_{3}\right), \widehat{H_{3}}$ comuta com $\left(1-e_{1}\right)$, logo

$$
e_{2}=\widehat{H}_{3}\left(1-e_{1}\right) \mathbf{e}
$$

Já, para $e_{3}$ :

$$
\begin{aligned}
e_{3}=\xi\left(\mathscr{A}_{\text {misto-3 }}\right) & =\left(1-e_{1}-e_{2}\right) \widehat{H}_{1}\left(2 g_{1}+g_{5}+g_{11}\right) \widehat{H}_{1}\left(1-e_{1}-e_{2}\right) \mathbf{e} \\
& =\left(2 g_{1}+g_{5}+g_{11}\right)\left(1-e_{1}-e_{2}\right) \mathbf{e}
\end{aligned}
$$

pois, novamente, temos um produto de idempotentes $e_{1}$ e $e_{2}$ que comutam com $\left(2 g_{1}+g_{5}+g_{11}\right)$

Por fim, $e_{4}$ possui endereço [1], ou seja,

$$
\begin{aligned}
e_{4} & =\left(1-e_{1}-e_{2}-e_{3}\right) \widehat{H}_{1}\left(1-e_{1}-e_{2}-e_{3}\right) \mathbf{e} \\
& =\left(1-e_{1}-e_{2}-e_{3}\right) \mathbf{e}
\end{aligned}
$$


Exemplo 2.16 Tomando $G_{[8,4]} \cong Q_{8}$, ainda em $\mathbb{F}_{3}$, temos $\mathbb{F} G \cong 4 \mathbb{F}_{3} \oplus M_{2}\left(\mathbb{F}_{3}\right)$.

Sendo $\mathbf{e}=1-\widehat{G^{\prime}}$, temos via HatMapXi

$$
\mathscr{A}=[[[1],[[3,1],[2,3],[2,5]]], 1]
$$

Ou seja

$$
\begin{aligned}
e_{1} & =\xi\left(\mathscr{A}_{\text {misto-1 }}\right)=\widehat{H}_{1}\left(2 g_{1}+g_{3}+g_{5}\right) \widehat{H}_{1} \mathbf{e} \\
& =\left(2 g_{1}+g_{3}+g_{5}\right) \mathbf{e}
\end{aligned}
$$

já $e_{2}=\xi([1])=\left(1-e_{1}\right) \mathbf{e}$.

Neste caso, ambos os idempotentes geram códigos $[8,2,6]$ sobre $\mathbb{F}_{3}$. Tal código corresponde a um Código Ótimo de acordo com [7]

Vemos uma descrição breve a seguir dos 10 primeiros grupos tais que $\mathbb{F}_{3} G$ seja não Abeliano. Na ordem: identificação do grupo, Estrutura, dimensão das componentes de Wedderburn, respectiva classificação dos idempotentes obtidos por endereços do tipo $\mathfrak{h}$, ou seja endereço Hat puro $(\mathrm{H})$, ou apresentando um endereço composto, ou seja, idempotentes descritos com pelo menos um endereço do tipo misto $(\mathrm{M})$

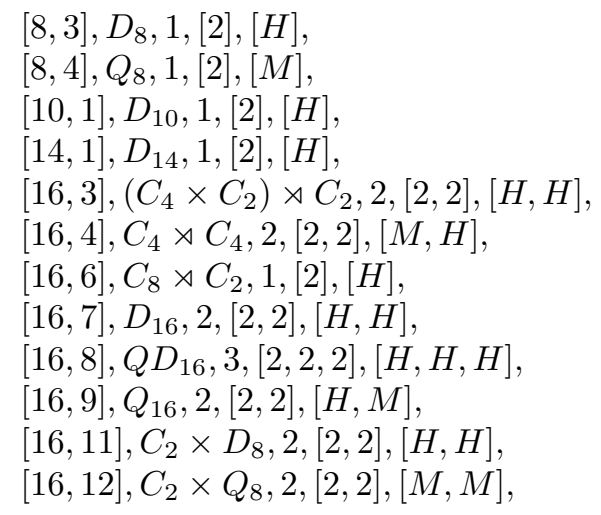

Ou seja, vemos que se $G \cong C_{2} \times D_{8}$ então $\mathbb{F}_{3} G$ possui 2 componentes simples $2 \times 2$ e ambas possuem um endereço $\mathfrak{h}$ de comprimento 2 . Uma análise em torno de todas as componentes de $\mathbb{F}_{3} G$ para álgebras de grupo finitas, semissimples e não Abelianas, para $G$ com ordem inferior a 200, excluindo-se grupos de ordem $2^{7}$ mostra uma relação de 3208 componentes marcadas como $H$ e 713 marcadas como $M$.

Seguem outros exemplos interessantes: 
$[196,8],\left(C_{7} \times C_{7}\right) \rtimes C 4,4,[4,4,4,4],[\mathrm{H}, \mathrm{H}, \mathrm{H}, \mathrm{H}]$,

$[200,40],\left(C_{5} \times C_{5}\right) \rtimes C_{8}, 3,[8,8,8],[\mathrm{H}, \mathrm{H}, \mathrm{H}]$,

[200,41 ], $D_{10} \times\left(C_{5} \rtimes C_{4}\right), 7,[2,2,4,2,2,4,8],[\mathrm{H}, \mathrm{H}, \mathrm{H}, \mathrm{H}, \mathrm{H}, \mathrm{H}, \mathrm{H}]$.

Tais grupos são exemplos de álgebras de grupo com diversas componentes de Wedderburn grande e todos os idempotentes primitivos de cada uma das coordenadas descritos completamente por endereços do tipo $\mathfrak{h}$. 


\section{Capítulo 3}

\section{Idempotentes em Álgebras de Grupo Não Semissimples}

Vamos agora utilizar nossos recursos para explorar exemplos em álgebras de grupo não semissimples. Porém, ao lidarmos com álgebras de grupo por um viés algorítmico-computacional, certas abordagens e certas classes de álgebras tornamse viáveis de serem estudadas, entretanto o tamanho de $\mathbb{F} G$ cresce de forma não linear em relação à ordem de G. Devemos portanto estar atentos à essa peculiaridade, sempre procurando minimizar a quantidade de recurso gasto.

Nossos algoritmos princiais dependem basicamente da pré-existência de idempotentes centrais primitivos bem como da estrutura conhecida de suas componentes de Wedderburn. Já, quando $\mathbb{F} G$ não é semissimples não dispomos de tais informações.

Uma boa estratégia para lidar com o caso não semissimples, é buscar previamente por idempotentes centrais quaisquer e fazer cisões na álgebra, quebrando-a em somas de ideais de dimensões menores. Em seguida realizamos um busca em uma base de seu centro $\mathcal{Z}(\mathbb{F} G)$ por idempotentes, e, em posse de uma lista suja de idempotentes centrais, ou seja, uma lista que possui idempotentes primitivos e somas de primitivos, decidiremos quais, de fato, são os primitivos.

\subsubsection{A Função de Competição}

Dentro de nossa abordagem é possível determinar todos os idempotentes de um anel de grupo finito $\mathbb{F} G$. Entretanto, o método pode ser demasiadamente custoso. Apesar disso, quando o objetivo é o de obter os idempotentes centrais (primitivos ou não) de $\mathbb{F} G$, uma alternativa relativamente viável, é examinar diretamente os 
idempotentes no centro de $\mathbb{F} G$, e, à partir daí, encontrar os idempotentes centrais primitivos.

Definição 3.1 Seja $E$ um subconjunto de idempotentes centrais de $\mathbb{F} G$ e seja $c$ : $E \times E \rightarrow \mathbb{F} G$ a função dada por:

$$
c\left(e_{1}, e_{2}\right)= \begin{cases}0 & , \text { se }_{1} \cdot e_{2}=e_{1} \text { ou } e_{1} \cdot e_{2}=0 \\ e_{1} & \text { casocontrário }\end{cases}
$$

Chamaremos $c$ de função de competição.

Ou seja, assim como definida, a função de competição possui o papel de exibir idempotentes não primitivos por sua imagem. Sua implementação consiste na função $\mathbf{C}\left(e_{1}, e_{2}, F G\right)$ a seguir:

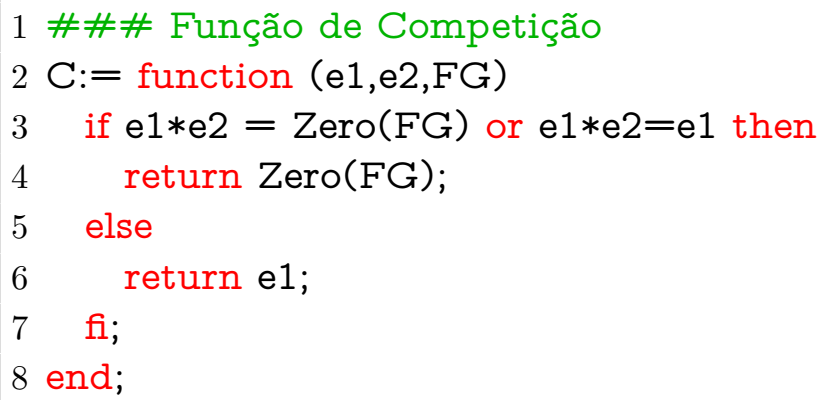

Proposição 3.2 Seja $\mathbb{F} G$ álgebra de grupo finita, e $E=\left\{e_{i}\right\}_{1 \leq i \leq n}$ um subconjunto de idempotentes centrais não triviais que contém todos os os idempotentes centrais primitivos de $\mathbb{F} G$. Seja $E_{1}=E$ e

$$
E_{i+1}=E_{i} \backslash \operatorname{Im} c\left(e_{i}, E_{i}\right), i \geq 1
$$

Então existe algum $m, m \leq n+1$ tal que

$$
\mathfrak{C} \stackrel{\text { def }}{=} E_{m}
$$

corresponde aos idempotentes centrais primitivos de $\mathbb{F} G$.

Demonstração: É claro que a proposição decorre do fato de que $e \in c(e, E)$ se, e somente se, $e$ não é um idempotente central primitivo. Primeiramente, notemos que se $e$ for de fato um idempotente central primitivo $c(e, E)=0$, assim, os idempotentes primitivos ficam preservados por cada iteração de $E_{i}$, ou seja $E_{n+1}$ 
contém todos os idempotentes primitivos. Além disso, suponha $e_{j_{1}}, . ., e_{j_{n_{1}}}$, sejam os $n_{1}$ idempotentes centrais primitivos, então as multiplicações $e e_{i^{\prime}}, 1 \leq i^{\prime} \leq n_{1}$ serão todas avaliadas. Se e não é um idempotente primitivo, então existe pelo menos algum $e_{j_{o}}$, idempotente central primitivo, em $E$ tal que $c\left(e, e_{j_{o}}\right)=e$, logo, $e$ será removido na próxima iteração, isto é, seja $k$, tal que $e_{k} \in E$, não seja um idempotente primitivo, $c\left(e_{k}, e_{i_{o}}\right)=e_{k} \in c\left(e_{k}, E_{k}\right) \operatorname{logo}, E_{k+1}=E_{k} \backslash c\left(e_{k}, E_{k}\right) \mathrm{e}$ portanto, $e_{k} \notin E_{k+1}$. Seja $n_{2}$ o número de idempotentes centrais não primitivos em $E$, então para todo $n^{\prime}>n_{1}+n_{2}=n$, segue que $E_{n^{\prime}}=\left\{e_{1}, \ldots, e_{n_{1}}\right\}$, logo, existe algum inteiro $m \leq n_{1}+n_{2}+1=n+1$ tal que $E_{m}$ corresponde aos idempotentes centrais primitivos de $\mathbb{F} G$.

Dessa forma, o conjunto $\mathfrak{C}$ pode ser encontrado por meio do algoritmo Challenge

1 \#\#\#\# Recebe uma lista de idempotentes centrais contendo os primitivos

2 \#\#\#\# retorna os primitivos

3

4 Challenge := function ( $E, F G$ )

5 \# Requer a função de competição

6 local Imc, \# Imagem da função de competição

7 Ei, \# conjuntos Ei

8 result, \#resultado confronto

$9 \quad \mathrm{i}, \mathrm{j} ;$ \# indices

10

$11 \mathrm{Ei}:=\mathrm{E} ;$ \# Carrega E1

12

13 for $i$ in [1..Size(E)] do \# c (ei, ej)

$14 \operatorname{Imc}:=[] ; \#$ esvazia imagem de $\mathrm{c}$

15 for $\mathrm{j}$ in $[1 . . \operatorname{Size}(\mathrm{Ei})]$ do

16 Add (Imc, C ( E[i] , Ei[j],FG ));

17 od;

18 Ei:= Filtered ( $\mathrm{Ei}, \mathrm{x}->$ not $(\mathrm{x}$ in Imc)); \# Carrega E_(i+1)

19 od; \# Ei sai com índice $n+1$;

20

21 return Ei;

22 end;

Porém, para obtermos os idempotentes centrais primitivos devemos assegurar que a lista $E$ os contenha antes de aplicarmos Challenge. Assim, vamos quebrar $\mathbb{F} G$ previamente em somas de ideais menores buscando primeiramente por idem- 
potentes centrais quaisquer. Em seguida, vamos reduzir esses ideais a uma base de seu centro, onde faremos nossa busca.

Assim, considerando o conjunto $N_{\mathfrak{h}}=\left\{\widehat{N}_{i}, N \text { subgrupo normal de } G\right\}_{\widehat{N}_{i} \neq 0}$ em $\mathbb{F} G$, caso não seja vazio, obtemos os primeiros idempotentes centrais. Seja $\left\{C_{i}\right\}_{i \in I}$ o conjunto das classes de conjugação de $G$ e $\gamma_{i}=\hat{C}_{i}=\sum_{x \in C_{i}} x$ suas respectivas somas de classes em $\mathbb{F} G$. Então, é sempre possível tomar uma potência $b$ de $\gamma_{i}$ de forma que $\gamma_{i}^{b}$ seja idempotente. De fato, seja $\gamma=\sum_{g \in C_{o}} g^{-1} a g$ uma soma de classe, em termos de um representante $a$ de uma classe $C$, e $C_{o}$ o conjunto dos elementos de $G$ que formam a classe $C$ por conjugação em $a$. Sendo $\operatorname{char}(F)=p$ e $o(a)=p^{k} m$ a ordem de $a$ onde $p \nmid m$, temos que $p^{\phi(m)} \equiv 1(\bmod m)$. Assim

$$
\begin{aligned}
\gamma^{p^{(\phi(m)+k)}} & =\sum_{g \in C_{o}} g^{-1} a^{p^{(\phi(m)+k)}} g \\
& =\sum_{g \in C_{o}} g^{-1} a^{p^{k}} g
\end{aligned}
$$

No entanto, quando obtemos elementos $\alpha$ de $\mathbb{F} G$ tais que $\alpha^{m}=\alpha$ para algum $m \in \mathbb{Z}, m>2$, temos que $\alpha^{m-1}$ é idempotente

$$
\begin{aligned}
\left(\alpha^{m-1}\right)^{2} & =\alpha^{2 m-2}=\alpha^{m} \alpha^{m-2} \\
& =\alpha \alpha^{m-2}=\alpha^{m-1}
\end{aligned}
$$

assim, temos que

$$
\begin{aligned}
\left(\gamma^{p^{(\phi(m)+k)}}\right)^{p^{\phi(m)}} & =\sum_{g \in C_{o}} g^{-1}\left(a^{p^{k}}\right)^{p^{\phi(m)}} g=\sum_{g \in C_{o}} g^{-1}\left(a^{p^{\phi(m)}}\right)^{p^{k}} g \\
& =\sum_{g \in C_{o}} g^{-1} a^{p^{k}} g=\gamma^{p^{(\phi(m)+k)}}
\end{aligned}
$$

Logo, tomando $\alpha=\gamma^{p^{(\phi(m)+k)}}$ e $m=p^{\phi(m)}$, segue que $\alpha^{m-1}=\gamma^{\left(p^{2 \phi(m)+k}-p^{\phi(m)+k}\right)}$ é idempotente. Ou seja, é sempre possível encontrar $b \leq\left(p^{2 \phi(m)+k}-p^{\phi(m)+k}\right)$ tal que $\gamma^{b}$ é um idempotente.

Caso $\gamma_{i}^{b}$ seja não trivial, obtemos outros idempotentes centrais assim, seja $\left\{\gamma_{i}^{b_{i}}\right\}_{I_{2}}$ o conjunto de tais idempotentes. Se $E_{o}=N_{\mathfrak{h}} \cup\left\{\gamma_{i}^{b_{i}}\right\}_{I_{2}}$ obtemos um conjunto de idempotentes centrais de $\mathbb{F} G$ tais que para cada $x_{i}$ tenhamos

$$
1=x_{i}+\left(1-x_{i}\right)
$$


a unidade escrita como soma de idempotentes centrais ortogonais. E portanto, para $x_{i}$ e $x_{j}$ em $E_{o}$,

$$
\begin{aligned}
1 & =1.1=\left(x_{i}+\left(1-x_{i}\right)\left(x_{j}+\left(1-x_{j}\right)\right)\right. \\
& =x_{i} x_{j}+x_{i}\left(1-x_{j}\right)+\left(1-x_{i}\right) x_{j}+\left(1-x_{i}\right)\left(1-x_{j}\right)
\end{aligned}
$$

Assim, os termos não nulos da última soma são também idempotentes centrais dois a dois ortogonais tais que sua soma é 1. Efetuando esses produtos para cada um dos elementos de $E_{o}$, e coletando todos os termos não nulos, obtemos um segundo conjunto de produtos $P$ de idempotentes centrais, dois a dois ortogonais tais que

$$
\sum_{e \in P} e=1
$$

Se o conjunto formado pelas somas de classes $\left\{\gamma_{i}\right\}$ formam uma base para $\mathcal{Z}(\mathbb{F} G)$ então, para cada elemento $e$ de $P$, podemos reduzir $\left\{\gamma_{i} e\right\}$ a uma base de $\mathcal{Z}(\mathbb{F G e})$.

Aplicando um método similar ao que retorna os endereços do tipo $\mathfrak{f}$ em cada uma das bases $\left\{\gamma_{i} e\right\}$, podemos utilizar uma função de crivo $C_{r}$ que captura os idempotentes de $\mathbb{F} G e$. Assim, determinamos um conjunto de idempotentes centrais $E$ de $\mathbb{F} G$ tal que $E$ contenha os idempotentes primitivos de $\mathbb{F} G$.

Podemos agora obter o conjunto $\mathfrak{C}$ obtido por sucessivas aplicações da função de competição. O método descrito traduz-se no algoritmo PCIbySOCI $(F G)$, em referência a Primitive Central Idempotents By Some Ortogonal Central Idempotents. O algoritmo descrito depende de funções SOCI(FG), Reduction2FBase e HunterEngine, presentes no último capítulo , além da função Challenge , já apresentada.

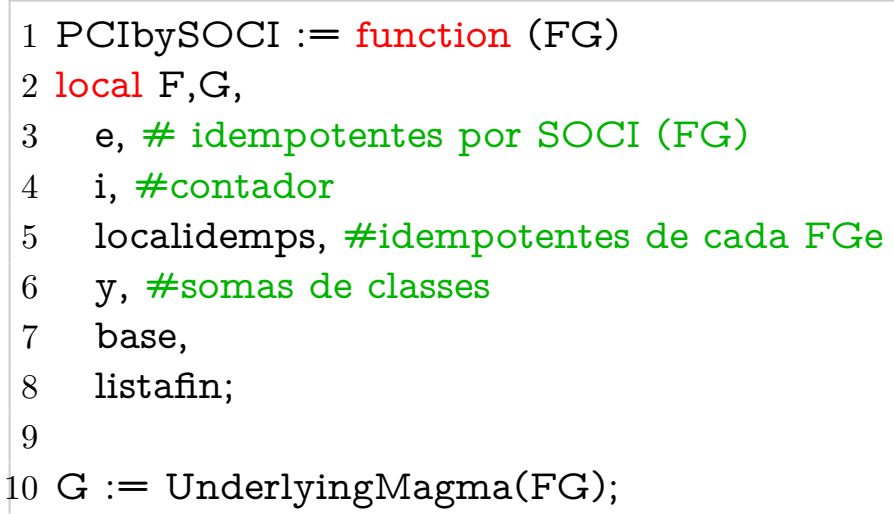


$11 \mathrm{~F}:=$ LeftActingDomain(FG);

12 localidemps :=[];

13 listafin:=[];

$14 \mathrm{e}:=\operatorname{SOCI}(\mathrm{FG})$;

$15 \mathrm{y}:=\mathrm{Y}(\mathrm{FG})$;

16

17 for $\mathrm{i}$ in [1...Size(e)] do

18 base: $=$ Reduction2FBase (FG, e[i]*y);

19 localidemps:= Set(HunterEngine (1, 1, 5000, FG, base, FG, " $x^{\wedge} 2-x^{\prime}$, $0,0,0)$ );

20 RemoveSet(localidemps, Zero(FG));

21 Add(listafin, localidemps);

22 localidemps: $=[]$;

23 od;

24 return Challenge (Flat(listafin),FG);

25 end;

Em $\mathbb{F}_{2} S_{5}$, obtemos dois únicos idempotentes centrais primitivos $e_{1}$ e $e_{2}$ tais que $e_{1}=1+(3,4,5)+(3,5,4)+(2,3,4)+(2,3,5)+(2,4,3)+(2,4,5)+(2,5,3)+(2,5,4)+$ $(1,2,3)+(1,2,3,4,5)+(1,2,3,5,4)+(1,2,4,5,3)+(1,2,4)+(1,2,4,3,5)+(1,2,5,4,3)+$ $(1,2,5)+(1,2,5,3,4)+(1,3,2)+(1,3,4,5,2)+(1,3,5,4,2)+(1,3,4)+(1,3,5)+(1,3,2,4,5)+$ $(1,3,5,2,4)+(1,3,2,5,4)+(1,3,4,2,5)+(1,4,5,3,2)+(1,4,2)+(1,4,3,5,2)+(1,4,3)+$ $(1,4,5)+(1,4,5,2,3)+(1,4,2,3,5)+(1,4,2,5,3)+(1,4,3,2,5)+(1,5,4,3,2)+(1,5,2)+$ $(1,5,3,4,2)+(1,5,3)+(1,5,4)+(1,5,4,2,3)+(1,5,2,3,4)+(1,5,2,4,3)+(1,5,3,2,4)$

e $e_{2}=1-e_{1}$.

Já, em $\mathbb{F}_{2} D_{18}$, com $D_{18}$ dado por

$$
D_{18}=<f_{1}, f_{2}, f_{3} \mid f_{1}^{2}=f_{3}^{3}=\left(f_{1} f_{2}^{-1}\right)^{2}=f_{2}^{2} f_{3}^{-1} f_{2}=1>
$$

obtemos três idempotentes $e_{1}, e_{2}$ e $e_{3}$ tais que

$$
\begin{aligned}
& e_{1}=1+f_{2}+f_{3}+f_{2}^{2}+f_{2} f_{3}+f_{3}^{2}+f_{2}^{2} f_{3}+f_{2} f_{3}^{2}+f_{2}^{2} f_{3}^{2} \\
& e_{2}=f_{2}+f_{2}^{2}+f_{2} f_{3}+f_{2}^{2} f_{3}+f_{2} f_{3}^{2}+f_{2}^{2} f_{3}^{2} \\
& e_{3}=1-e_{1}-e_{2}
\end{aligned}
$$

Para uma álgebra de grupo não semissimples $\mathbb{F} G$, não podemos afirmar que para cada e, idempotente central, $\mathbb{F} G$ e seja isomorfo a uma álgebra de matrizes, porém, podem eventualmente ocorrer casos em que $\mathbb{F} G \mathbf{e}$, de fato, o seja. 


\subsubsection{Detecção de Componentes Simples}

Uma vez determinados os idempotentes centrais primitivos de $\mathbb{F} G$, podemos estudar a possibilidade de decompor esses idempotentes centrais em idempotentes primitivos pela aplicação de HatMapXi . Em caso positivo, isto é, suponha haver um endereço $\mathscr{A}$ de $\mathscr{M}(\mathbf{e})$, e um idempotente central primitivo de $\mathbb{F} G$ em que $\mathscr{A}$ descreva idempotentes $e_{1}, \ldots, e_{n}$, dois a dois ortogonais com dimensão sobre $\mathbb{F}$ igual a $q=p^{k}$ para algum $q$.

Se conseguirmos determinar elementos do tipo $e_{i j}$ tais que $e_{i j} \cdot e_{r s}=e_{i s} . \delta(j, r)$ para todo $i, j, r, s \in[1 . . n]$ fica fácil estabelecer um isomorfismo $\phi: M_{n}\left(\mathbb{F}_{q}\right) \rightarrow \mathbb{F} G \mathbf{e}$ via $\phi\left(\mathrm{E}_{i, j}\right)=e_{i j}$, onde $\mathrm{E}_{i, j}$ denotam as matrizes elementares de $M_{n}\left(\mathbb{F}_{q}\right)$.

Mesmo em posse dos idempotentes $e_{1}, . ., e_{n}$ e do respectivo endereço $\mathscr{A}$ da família, obter tais elementos $e_{i j}$ não é tarefa simples. Porém, o próximo algoritmo busca pela existência de uma matriz $M$, a qual chamaremos de matriz Pivot, do morfismo $\phi$ com a seguinte propriedade:

$$
\left(e_{i} \cdot M_{i j} \cdot e_{j}\right)\left(e_{r} \cdot M_{r s} \cdot e_{s}\right)=\left(e_{i} \cdot M_{i s} \cdot e_{s}\right) \delta(j, r)
$$

para todo $i, j, r, s \in[1 . . n]$.

A existência de tal matriz nos permite definir os elementos da forma $e_{i j}$ como

$$
e_{i j} \stackrel{d e f}{=} e_{i} \cdot M_{i j} \cdot e_{j}
$$

Dessa forma, $e_{i j}$ possui as propriedades almejadas. A seguinte sequência de algoritmos objetiva a busca por matrizes Pivot de uma família de idempotentes primitivos de $\mathbb{F} G \mathbf{e}$ para o caso em que temos $\operatorname{dim} e_{i} \mathbb{F} G \mathbf{e} e_{j}=1$ sobre $\mathbb{F}$ para cada par de idempotentes primitivos $e_{i}$ e $e_{j}$. Para o caso em que $\mathbb{F}$ é corpo de decomposição, apresentamos uma sequência de algoritmos que viabilizam alguns desses morfismos.

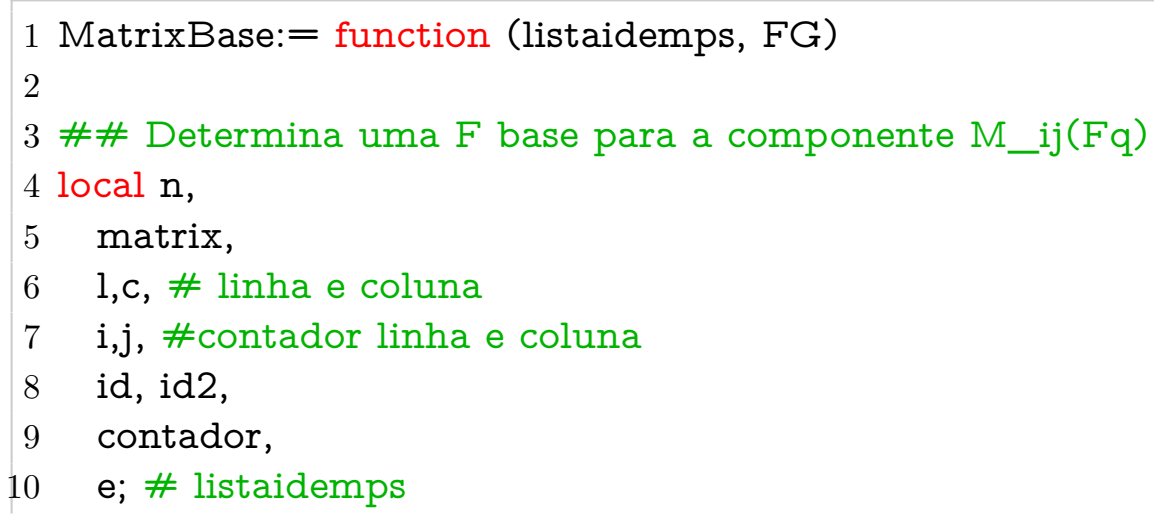


$11 \mathrm{n}:=$ Size(listaidemps);

12 matrix $:=[]$;

$13 \mathrm{l}:=[]$;

14 e:=listaidemps;

$15 \mathrm{G}:=$ UnderlyingMagma(FG);

$16 \mathrm{~F}:=$ LeftActingDomain(FG);

17 for $i$ in [1..n] do

18 for $\mathrm{j}$ in [1..n] do

19 id:= Reduction2FBase (FG, e[i]*List $(G) * e[j])[1]$;

20 if $i=j$ then

21 id2:=id;

22 while id2 $2<$ id2 or id2 $=\operatorname{Zero}(F G)$ do

23 id2 $:=$ id $*$ Random $(F)$;

24 od;

25 id: $=i d 2$;

26 fi;

$27 \quad$ Add (l, id ) ;

28 od;

29 Add(matrix, 1);

$30 \quad \mathrm{l}:=[\mathrm{]}$

31 od;

32 \#\#\# Correção Triangular Inferior:

$33 \mathrm{i}:=1$;

$34 \mathrm{j}:=1$;

35 Print ("\n Executando correção Triangular: \n");

36 contador: $=[i, j]$;

37 Print ( "Contador: ", contador);

38 for $\mathrm{j}$ in [1..n] do \#coluna

39 contador: $=[i, j]$;

40 Print ( "Contador: ", contador);

41 Print (" \n iniciando Coluna ", j);

42 for $i$ in [j..n] do \#linha

43 contador: $=[i, j]$;

$44 \quad \mathrm{id}:=$ matrix $[\mathrm{i}, \mathrm{j}]$;

45 id2:=id;

46 while id2*matrix $[\mathrm{j}, \mathrm{i}]$ < $\operatorname{matrix}[\mathrm{i}, \mathrm{i}]$ do

$47 \quad$ id2 $:=\mathrm{id} *$ Random $(F)$;

48 contador $:=[i, j]$;

49 od;

$50 \quad \operatorname{matrix}[i, j]:=i d 2$; 
51 od;

52 od;

53 return matrix;

54 end;\#\#\#\#\#\#\#\#\#\#\#\#\#\#\#\#\#\#\#\#

55

56 OneMatCorrection :=function (Mat,FG,NmexerLista) 57

58 local n, \#número de prods c erro

59 q, \# contador

$60 \mathrm{M}$, \# copia da matrix

61 Eik,

62 Ekj,

63 Eij,

64 fpos,

65 a,b,c, \#congelados

66 erros;

67

68

$69 \mathrm{~F}:=\operatorname{Separa}(F G)[1]$

$70 \mathrm{G}:=\operatorname{Separa}(F G)[2]$;

71

72 erros:= MatrixTester (Mat,FG);

$73 \mathrm{n}:=$ Size(erros);

$74 \mathrm{M}:=$ IdentityMat(Size(Mat),FG)*Mat;

75 \#\#Corrigindo

76

77 for $\mathrm{q}$ in [1..n] do

78 Eik := Mat [ erros[q][1][1], $\operatorname{erros[q][1][2]];~}$

$79 \operatorname{Ekj}:=$ Mat [ erros[q][2][1], erros[q][2][2]];

80 Eij := Mat [ erros[q][1][1], erros[q][2][2]];

$81 \mathrm{a}:=[\operatorname{erros}[q][1][1], \operatorname{erros}[q][1][2]] ;$

$82 \mathrm{~b}:=[\operatorname{erros}[q][2][1], \operatorname{erros}[q][2][2]] ;$

$83 \mathrm{c}:=[\operatorname{erros}[q][1][1], \operatorname{erros}[q][2][2]] ;$

84

85 if not (erros[q][1] in NmexerLista) then \#arrumar Eik

86 fpos:=Position ( List $(F, x->(x * E i k) *(E k j)=E i j)$, true );

87

$88 \mathrm{M}[\operatorname{erros}[q][1][1], \operatorname{erros}[q][1][2]]:=\operatorname{List}(F)[f p o s] *$ Eik;

89 Print ("\n \n Return tipo 1");

$90 \operatorname{return}[\mathrm{M},[\operatorname{erros}[\mathrm{q}][1][1], \operatorname{erros}[\mathrm{q}][1][2]$ ] , a,b,c]; 


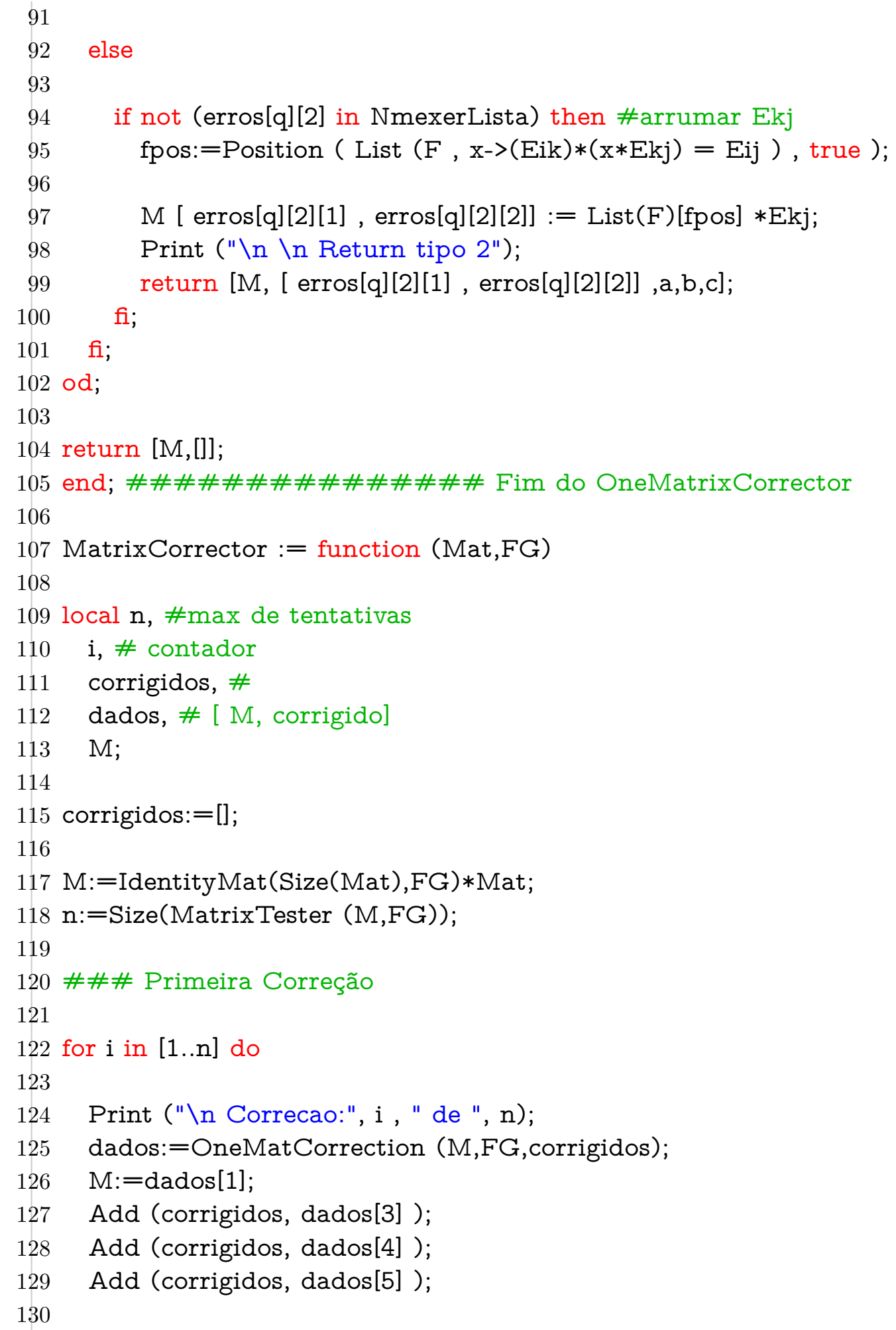




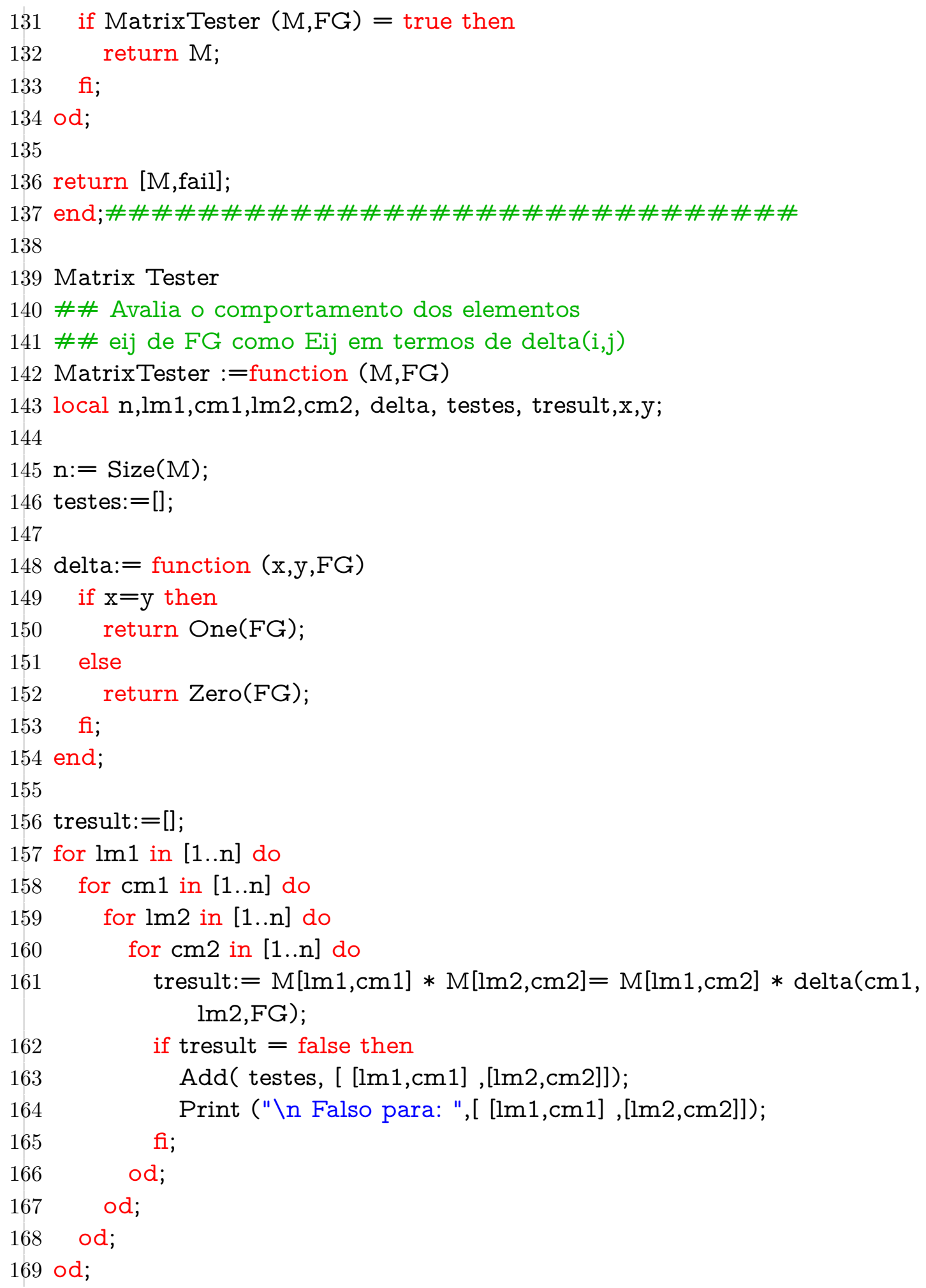


170 Print (" \n \n");

171

172 if Set(testes) $=[]$ then

173 return true;

174 else

175 return Set(testes);

176 fi;

177 end;

178

179 \#\#\#\#\#\#\#\#\#\#\#\#\#\#\#\#\#\#\#\#

180 \#\# dado UM ÚNICO ELEMENTO elem retorna [alpha,g ] tal que elem $=$ eii $\cdot$ alpha $\cdot g \cdot$ e jj

181 \# EiiEjj $=[$ eii,ejj]

182

183 IdentifyMe := function (elem, EiiEjj, FG)

184

185 local F,G, eii,ejj, i,j;

186

187 eii:=EiiEjj[1];ejj:=EiiEjj[2];

188

$189 \mathrm{~F}:=\operatorname{Separa}(F G)[1] ; G:=\operatorname{Separa}(F G)[2] ; i:=1 ;$

190

191 while eii*List(G)[i]*ejj $=\operatorname{Zero}(F G)$ do

192 i : = i+1;

193 od;

$194 \mathrm{j}:=1$;

195 while List(F)[j]*eii*List(G)[i]*ejj $<>$ elem do

$196 \mathrm{j}:=\mathrm{j}+1$;

197 od;

198

199 return One(FG)*List(G)[i] $* \operatorname{List}(F)[j]$;

200 end;

201

202 Por fim, o algoritmo busca pela existência de matrizes \$Pivot\$, 203

$204 \backslash$ begin $\{$ lstlisting $\}$ [language $=$ GAP]

205 \#\# Dada uma Matriz nxn de FG do tipo Mij,

206 \#\# Rotorna a Matriz de Reconhecimento

207

208 IdentifyMat := function (M, Idemps, FG) 


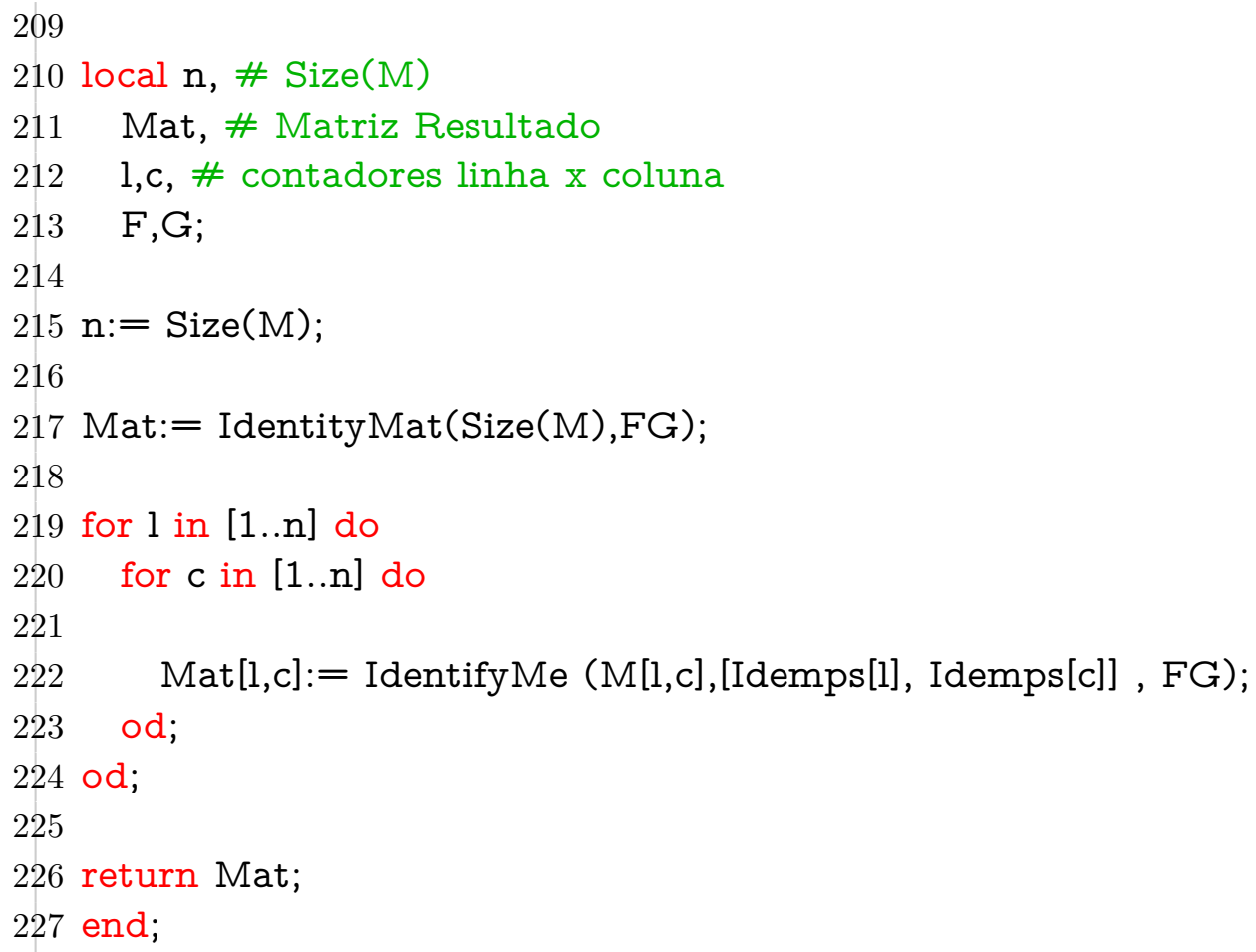

Exemplo 3.3 Considere $F_{5} S_{5}$. Sejam $\mathbf{e}_{1}, \mathbf{e}_{2}$ e $\mathbf{e}_{3}$ os idempotentes centrais primitivos obtidos por PCIBySOCI(FG) . Temos que $\mathbf{e}_{1}$ e $\mathbf{e}_{2}$ são idempotentes que possuem o seguinte endereço para seus respectivos idempotentes primitivos não centrais.

$$
\begin{aligned}
& \mathscr{A}_{\mathbf{e}_{1}}=[27,58,6,10,1] \text { com } \operatorname{dim} \mathbb{F} G \mathbf{e}_{\mathbf{1}}=25 . \text { Onde } \\
& \qquad \begin{aligned}
H_{27} & =<(2,5,4)>, H_{58}=<(1,3),(2,4)>, \\
H_{6} & =<(2,4)>, H_{10}=<(1,3)>, H_{1}=<()>
\end{aligned}
\end{aligned}
$$

Já $\mathscr{A}_{e_{2}}=[27,45,2,7,1]$ com, também com $\operatorname{dim} \mathbb{F} G \mathbf{e}_{2}=25$ onde,

$$
\begin{gathered}
H_{45}=<(2,4,3,5)>, \\
H_{2}=<(4,5)>, \text { e } H_{7}=<(2,3)>
\end{gathered}
$$


por fim, $e_{3}=1-e_{1}-e_{2}$, com $\operatorname{dim} \mathbb{F} G e_{3}=70$ não será estudado.

Para $\mathbb{F}_{5} S_{5} \mathbf{e}_{\mathbf{1}}$ :

Considere $M$, a seguinte matriz de dimensão $5 \times 5$

$$
M=\left[\begin{array}{lllll}
(1) & 4(3,4) & 4(3,4) & 4(3,4) & 2(3,4) \\
3(3,4) & (1) & 3(3,4,5) & 4(4,5) & 2(3,4) \\
4(3,4) & 4(3,4,5) & (1) & 2(3,4) & 2(4,5) \\
4(3,4) & 2(4,5) & 2(3,4) & (1) & 2(3,4) \\
4(3,4) & 2(3,4) & 4(4,5) & 4(3,4) & (1)
\end{array}\right]
$$

então, definindo os elementos $e_{i j}$ por

$$
e_{i j} \stackrel{\text { def }}{=} e_{i} \cdot M_{i j} \cdot e_{j}
$$

são tais que $e_{i j} \cdot e_{r s}=e_{i s} . \delta(j, r)$

Onde

$$
\delta(x, y)= \begin{cases}1_{F G} & \text { se } x=y \\ 0_{F G} & \text { se } x \neq y\end{cases}
$$

Para $\mathbb{F}_{5} S_{5} \mathbf{e}_{2}$ :

Considere $N_{5 \times 5}$ a matriz:

$$
N=\left[\begin{array}{lllll}
(1) & 3(1,2) & 3(3,4) & 4(3,4) & 3(1,2)(3,4) \\
3(1,2) & (1) & 3(1,2) & 4(1,2)(3,4) & (3,4) \\
4(3,4) & 4(1,2) & (1) & 2(3,4) & 4(1,2)(3,4) \\
(3,4) & (1,2)(3,4) & (3,4) & (1) & 2(1,2) \\
4(1,2)(3,4) & 3(3,4) & 4(1,2)(3,4) & 4(1,2) & (1)
\end{array}\right]
$$

então, definindo os elementos $e_{i j}$ por

$$
e_{i j} \stackrel{\text { def }}{=} e_{i} \cdot N_{i j} \cdot e_{j}
$$


são tais que $e_{i j} \cdot e_{r s}=e_{i s} . \delta(j, r)$.

Como $M$ e $N$ são matrizes Pivot,ambas de dimensão $5 \times 5$, então há em $\mathbb{F}_{5} S_{5}$ duas componentes isomorfas a $M_{5}\left(\mathbb{F}_{5}\right)$, ou seja $M_{5}\left(\mathbb{F}_{5}\right) \hookrightarrow \mathbb{F}_{5} S_{5}$. Ambas as matrizes $M$ e $N$ foram obtidas através da execução de IdentifyMat. 


\section{Capítulo 4}

\section{Uma Aplicação para Idempotentes de QG}

\subsection{Reconhecimento de Idempotentes Centrais Primitivos em QG}

Uma vez percebida a relevância das decomposições do tipo hat para os idempotentes primitivos de $\mathbb{F} G$, a aplicabilidade desses algoritmos em $\mathbb{Q} G$ torna-se uma pergunta natural que emerge neste contexto. Com pequenas alterações nas funções de crivo $C_{r}$, podemos utilizar nossos algoritmos para reconhecer idempotentes centrais primitivos pré-calculados em termos de endereços do tipo hy. Isto é, suponha $G$ tal que seja conhecido o conjunto $I_{c p}$ dos idempotentes centrais primitivos de $\mathbb{Q} G$, definindo a função $C_{r}^{I c p \mathbb{Q}}$

$$
\begin{aligned}
C_{r}^{I c p \mathbb{Q}}: L \subset \mathbb{Q} G & \longrightarrow \\
\alpha & \longrightarrow \begin{cases}\alpha & \text { se } \alpha \in I_{c p} \\
0 & \text { se } \alpha \notin I_{c p}\end{cases}
\end{aligned}
$$

e, executando-se HatMapXi, obtemos possíveis endereços Hat para seus idempotentes. Por exemplo para $G_{[27,4]} \cong C_{9} \rtimes C_{3}$, temos que um endereço para os idempotentes centrais primitivos de $\mathbb{Q} G$ é

$$
\mathscr{A}=[10,9,8,7,6,-10]
$$

ou seja, $e_{1}=\widehat{H}_{10}$ 


$$
\begin{aligned}
& e_{1}=\xi\left(\left.\mathscr{A}\right|_{1}\right)=\widehat{H}_{10}=\widehat{G} \\
& e_{2}=\xi\left(\left.\mathscr{A}\right|_{2}\right)=\widehat{H}_{9}\left(1-e_{1}\right) \\
& e_{3}=\xi\left(\left.\mathscr{A}\right|_{3}\right)=\widehat{H}_{8}\left(1-e_{1}-e_{2}\right) \\
& e_{4}=\xi\left(\left.\mathscr{A}\right|_{4}\right)=\widehat{H}_{7}\left(1-e_{1}-e_{2}-e_{3}\right) \\
& e_{5}=\xi\left(\left.\mathscr{A}\right|_{5}\right)=\widehat{H}_{6}\left(1-e_{1}-e_{2}-e_{3}-e_{4}\right) \\
& e_{6}=\xi\left(\left.\mathscr{A}\right|_{6}\right)=\left(1-\widehat{H}_{10}\right)\left(1-e_{1}-e_{2}-e_{3}-e_{4}\right)=\left(1-e_{1}-e_{2}-e_{3}-e_{5}\right)
\end{aligned}
$$

Já, para $G_{[27,3]} \cong\left(C_{3} \times C_{3}\right) \rtimes C_{3}$, temos o endereço $\mathscr{A}=[19,18,17,16,15,-19]$. Ou seja,

$$
\begin{aligned}
e_{1}=\xi\left(\left.\mathscr{A}\right|_{1}\right) & =\widehat{H}_{19}=\widehat{G} \\
e_{2}=\xi\left(\left.\mathscr{A}\right|_{2}\right) & =\widehat{H}_{18}\left(1-e_{1}\right) \\
e_{3}=\xi\left(\left.\mathscr{A}\right|_{3}\right) & =\widehat{H}_{17}\left(1-e_{1}-e_{2}\right) \\
e_{4}=\xi\left(\left.\mathscr{A}\right|_{4}\right) & =\widehat{H}_{16}\left(1-e_{1}-e_{2}-e_{3}\right) \\
e_{5}=\xi\left(\left.\mathscr{A}\right|_{5}\right) & =\widehat{H}_{15}\left(1-e_{1}-e_{2}-e_{3}-e_{4}\right) \\
e_{6} & =\xi\left(\left.\mathscr{A}\right|_{6}\right)=\left(1-\widehat{H}_{19}\right)\left(1-e_{1}-e_{2}-e_{3}-e_{4}\right)=\left(1-e_{1}-e_{2}-e_{3}-e_{5}\right)
\end{aligned}
$$

Uma primeira análise nos fornece a seguinte tabela:

$\begin{array}{cclccl}\text { IdGrupo } & \text { Estrutura } & \text { Endereço } & \text { IdGrupo } & \text { Estrutura } & \text { Endereço } \\ {[1,1]} & 1 & {[1]} & {[8,3]} & D_{8} & {[10,9,8,7,-10]} \\ {[2,1]} & C_{2} & {[2,-2]} & {[8,4]} & Q_{8} & {[6,5,4,3,-6]} \\ {[3,1]} & C_{3} & {[2,-2]} & {[8,5]} & C_{2} \times C_{2} \times C_{2 C} & {[16,15,14, \ldots, 10,-16]} \\ {[4,1]} & C_{4} & {[3,2,-3]} & {[9,1]} & C_{9} & {[3,2,-3]} \\ {[4,2]} & C_{2} \times C_{2} & {[5,4,3,-5]} & {[9,2]} & C_{3} \times C_{3} & {[6,5,4,3,-6]} \\ {[5,1]} & C_{5} & {[2,-2]} & {[10,1]} & D_{10} & {[8,7,-8]} \\ {[6,1]} & S_{3} & {[6,5,-6]} & {[10,2]} & C_{10} & {[4,3,2,-4]} \\ {[6,2]} & C_{6} & {[4,3,2,-4]} & {[11,1]} & C_{11} & {[2,-2]} \\ {[7,1]} & C_{7} & {[2,-2]} & {[12,1]} & C_{3} \rtimes C_{4} & {[8,7,3,2,-8]} \\ {[8,1]} & C_{7} & {[2,-2]} & {[12,2]} & C_{12} & {[6,5,4,3,2,-6]} \\ {[8,2]} & C_{4} \times C_{4} & {[8,7,6,5,4,-8]} & {[12,3]} & A_{4} & {[10,9,-10]}\end{array}$

Analisando todos os grupos com ordem igual ou inferior a 120, percebe-se que dos 1237 grupos, 1143 possuem um endereço completo para seus idempotentes centrais primitivos. Desta análise, um dos maiores endereços ocorre em $G \cong\left(C_{2} \times C_{2} \times\right.$ $\left.C_{2} \times C_{2} \times C_{2} \times C_{2}\right)=G_{[64,267]}$, onde $\mathscr{A}=[2825,2824,2823, \ldots, 2762,2763,-2825]$ de tamanho 64, neste caso, $\mathbb{Q} G$ é uma álgebra de grupo comutativa. 


\subsection{HatMaps em QG}

Uma vez obtido um endereço $\mathfrak{h}$ para os idempotentes centrais primitivos de $\mathbb{Q} G$, podemos estudar seus idempotentes centrais primitivos por meio de HatMapXi e comparar seus os endereços dos idempotentes centrais primitivos com os idempotentes primitivos não centrais da respectiva componente simples.

Consideremos novamente $G=G_{[27,4]} \cong C_{9} \rtimes C_{3}$. Em $\mathbb{Q} G$, novamente sua única componente não comutativa é dada por $\mathbb{Q} G \mathbf{e}$ com $\mathbf{e}=\left(1-\widehat{G}^{\prime}\right)$, novamente com dimensão 18 sobre $\mathbb{Q}$. Temos o mesmo HatMap

$$
\begin{array}{r}
\mathscr{M}(\mathbf{e}, 3)=[[3,4,1],[3,4,5],[3,5,1],[3,5,4],[4,3,1],[4,3,5], \\
\\
[4,5,1],[4,5,3],[5,3,1],[5,3,4],[5,4,1],[5,4,3]]
\end{array}
$$

que a componente não comutativa de $\mathbb{F}_{2} G$ possui.

Ou seja, sendo $\mathscr{A}_{C e n}$ um endereço $H$ at dos idempotentes centrais primitivos e

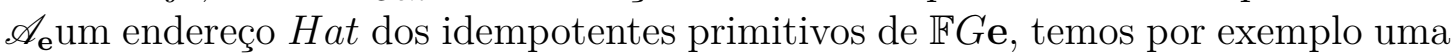
possível combinação de endereços.

$$
\mathscr{A}_{C e n}=[10,9,8,7,6,1] \text { e } \mathscr{A}_{\mathbf{e}}[5,4,3]
$$

A saber, sua disposição no reticulado de $\mathrm{G}$, configura-se como:

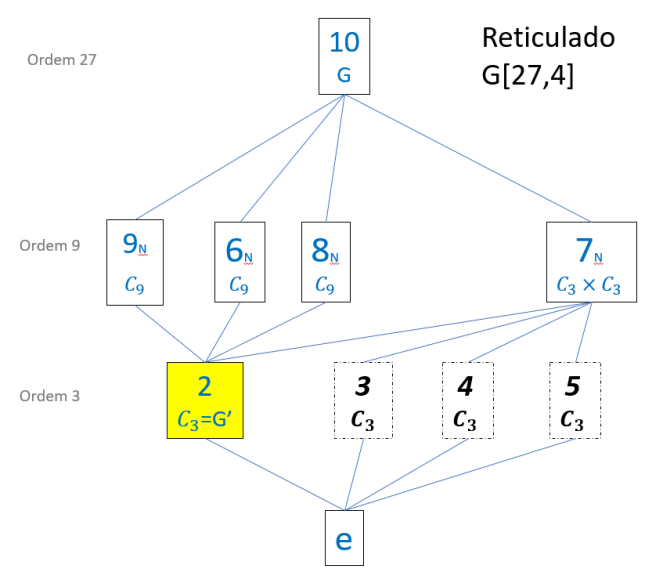

Onde os grupos marcados com $N$ são subgrupos normais de $G$. 


\section{Capítulo 5}

\section{Exemplos}

Exemplo 5.1 Para $\mathbb{F}_{3} D_{10}$, temos

$$
\mathbb{F}_{3} D_{10} \cong \mathbb{F}_{3} \oplus \mathbb{F}_{3} \oplus M_{2}\left(\mathbb{F}_{9}\right)
$$

Sendo $\mathbf{e}=\left(1-\widehat{G^{\prime}}\right)$ seu único idempotente referente à componente não comutativa, temos que

HatMapXi retorna o endereço $\mathscr{A}=[2,1]$ para esta componente. Ou seja,

$$
e_{1}=\xi\left(\left.\mathscr{A}\right|_{1}\right)=\widehat{H}_{2} \mathbf{e}
$$

enquanto

$$
e_{2}=\xi\left(\left.\mathscr{A}\right|_{2}\right)=\left(1-e_{1}\right) \mathbf{e}
$$

onde $H_{2}=<b>$, com $D_{10}=<a, b \mid a^{5}=b^{2}=b a b a=1>$

Exemplo 5.2 (Construção de idempotentes primitivos em $\mathbb{F}_{5} S_{3}$ )

Para $\mathbb{F}_{5} S_{3}$, temos:

$$
\mathbb{F}_{5} S_{3} \cong \mathbb{F}_{5} \oplus \mathbb{F}_{5} \oplus M_{2}\left(\mathbb{F}_{5}\right)
$$

Novamente, sendo $\mathbf{e}=\left(1-\widehat{G}^{\prime}\right)$, temos um endereço $\mathscr{A}=[2,1]$. Ou seja, 


$$
\begin{aligned}
& e_{1}=\xi\left(\left.\mathscr{A}\right|_{1}\right)=\widehat{H}_{2} \mathbf{e} \\
& e_{2}=\xi\left(\left.\mathscr{A}\right|_{2}\right)=\left(1-e_{1}\right) \mathbf{e}
\end{aligned}
$$

Tais idempotentes fornecem códigos do tipo $[6,2,4]$ sobre $\mathbb{F}_{5}$.

\section{Exemplo 5.3 (Construção de Código Ótimo $[12,3,8]$ em $\mathbb{F}_{5} A_{4}$ por meio de endereço misto)}

Considere $\mathbb{F}_{5} A_{4}$, para o endereço endereço $\mathscr{A}=[5$, [[2], [[2, 1]]], no caso, o segundo idempotente constroi um código ótimo $[12,3,8]$, segundo [7].

Com $A_{4}=<a, b \mid a^{3}=b^{3}=b a b a=1>$ :

Sendo $H_{5}=<b>$ e $H_{2}=<b a>$. e o idempotente dado por

$$
\begin{aligned}
e_{2} & =\left(1-\widehat{H}_{5}\right) \widehat{H}_{2}\left(1-\widehat{H}_{5}\right) 1\left(1-\widehat{H}_{5}\right) \widehat{H}_{2}\left(1-\widehat{H}_{5}\right) \mathbf{e} \\
& =\left(1-\widehat{H}_{5}\right) \widehat{H}_{2}\left(1-\widehat{H}_{5}\right)^{2} \widehat{H}_{2}\left(1-\widehat{H}_{5}\right) \mathbf{e} \\
& =\left(\widehat{H}_{2}-\widehat{H}_{5} \widehat{H}_{2}\right)\left(1-\widehat{H}_{5}\right)^{2}\left(\widehat{H}_{2}-\widehat{H}_{2} \widehat{H}_{5}\right) \mathbf{e} \\
& =\left(\widehat{H}_{2}-\widehat{H}_{5} \widehat{H}_{2}\right)\left(1-\widehat{H}_{5}\right)\left(\widehat{H}_{2}-\widehat{H}_{2} \widehat{H}_{5}\right) \mathbf{e}
\end{aligned}
$$

Ou então, utilizando a notação $\widehat{H}_{-5}=\left(1-\widehat{H}_{5}\right)$, temos que $e_{2}=\left(\widehat{H}_{-5} \widehat{H}_{2}\right)^{2} \widehat{H}_{-5} \mathbf{e}$

Pode-se obter códigos ótimos de acordo com [7] por meio das técnicas utilizadas.

\section{Exemplo 5.4 (Construção de Código Ótimo $[21,3,12]$ em $\mathbb{F}_{2}$ )}

Em $\mathbb{F}_{2}$, considere $G=G_{[21,1]} \cong C_{7} \rtimes C_{3}$. Assim, temos $G$ descrito por

$$
G=<f_{1}, f_{2} \mid f_{1}^{3}=1, f_{1} f_{2}^{2}=f_{2} f_{1}>
$$

então temos em $\mathbb{F}_{2} G_{[21,1]}$ a seguinte estrututa

$$
\mathbb{F}_{2} G \cong \mathbb{F}_{2} \oplus \mathbb{F}_{4} \oplus M_{3}\left(\mathbb{F}_{2}\right) \oplus M_{3}\left(\mathbb{F}_{2}\right)
$$

Onde $\mathbf{e}_{1}=1+f_{2}^{3}+f_{2}^{5}+f_{2}^{6}$ e $\mathbf{e}_{2}=1+f_{2}+f_{2}^{2}+f_{2}^{4}$ são os idempotentes centrais primitivos correspondentes às duas respectivas componentes não comutativas $M_{3}\left(\mathbb{F}_{2}\right)$. 
Executando-se HatMapXi $(1, \mathbf{e}, \mathbb{F} G)$, tanto para $\mathbf{e}=\mathbf{e}_{\mathbf{1}}$ quanto para $\mathbf{e}=\mathbf{e}_{2}$, obtém-se o endereço $[2,3,1]_{\mathfrak{h}}$

Em ambas as componentes, o mesmo endereço $\mathscr{A}=[2,3,1]_{\mathfrak{h}}$ retorna idempotentes primitivos de $\mathbb{F} G \mathbf{e}_{1}$ e de $\mathbb{F} G \mathbf{e}_{2}$ todos correspondentes a códigos ótimos do tipo $[21,3,12]$ sobre $\mathbb{F}_{2}$.

Os idempotentes de $\mathbb{F}_{2} G \mathbf{e}_{1}$ são:

$$
\begin{aligned}
& e_{1}=1+f_{1}+f_{1}^{2}+f_{2}^{3}+f_{1} f_{2}^{3}+f_{1}^{2} f_{2}^{3}+f_{2}^{5}+f_{1} f_{2}^{5}+f_{2}^{6}+f_{1}^{2} f_{2}^{5}+f_{1} f_{2}^{6}+f_{1}^{2} f_{2}^{6} \\
& e_{2}=1+f_{1}^{2}+f_{1} f_{2}+f_{2}^{2}+f_{1}^{2} f_{2}+f_{1} f_{2}^{2}+f_{2}^{3}+f_{1} f_{2}^{3}+f_{2}^{4}+f_{1}^{2} f_{2}^{4}+f_{1} f_{2}^{6}+f_{1}^{2} f_{2}^{6} \\
& e_{3}=1+f_{1}+f_{1} f_{2}+f_{2}^{2}+f_{1}^{2} f_{2}+f_{1} f_{2}^{2}+f_{2}^{3}+f_{2}^{4}+f_{1}^{2} f_{2}^{3}+f_{1}^{2} f_{2}^{4}+f_{1} f_{2}^{5}+f_{1}^{2} f_{2}^{5}
\end{aligned}
$$

, ou então, utilizando o entereço $\mathscr{A}$ visto como endereço do mapa $\mathscr{M}\left(\mathbf{e}_{1}\right)$, simplesmente,

$$
\begin{aligned}
e_{1} & =\xi\left(\left.\mathscr{A}\right|_{1}\right)=\xi([2])=\widehat{H}_{2} \mathbf{e}_{\mathbf{1}} \\
e_{2} & =\xi\left(\left.\mathscr{A}\right|_{2}\right)=\xi([2,3]) \\
& =\left(1-e_{1}\right) \widehat{H}_{3}\left(1-e_{1}\right) \mathbf{e}_{\mathbf{1}} \\
& =\widehat{H}_{3}\left(1-e_{1}\right) \mathbf{e}_{\mathbf{1}} \\
e_{3} & =\xi(\mathscr{A})=\xi([2,3,1]) \\
& =\left(1-e_{1}-e_{2}\right) \widehat{H}_{1}\left(1-e_{1}-e_{2}\right) \mathbf{e}_{\mathbf{1}} \\
& =\widehat{H}_{1}\left(1-e_{1}-e_{2}\right) \mathbf{e}_{\mathbf{1}}
\end{aligned}
$$

Já, se utilizarmos $\mathscr{A}$ como mapa de $\mathscr{M}\left(\mathbf{e}_{2}\right)$, então os idempotentes serão

$$
\begin{aligned}
e_{1} & =\xi\left(\left.\mathscr{A}\right|_{1}\right)=\xi([2])=\mathbf{e}_{2} \widehat{H}_{2} \\
e_{2} & =\xi\left(\left.\mathscr{A}\right|_{2}\right)=\xi([2,3]) \\
& =\left(1-e_{1}\right) \widehat{H}_{3}\left(1-e_{1}\right) \mathbf{e}_{2} \\
e_{3} & =\xi(\mathscr{A})=\xi([2,3,1]) \\
& =\left(1-e_{1}-e_{2}\right) \widehat{H}_{1}\left(1-e_{1}-e_{2}\right) \mathbf{e}_{2} \\
& =\left(1-e_{1}-e_{2}\right) \mathbf{e}_{2}
\end{aligned}
$$


Sendo $H_{1}=(1), H_{2}=<f_{1}>$ e $H_{3}=<f_{1} f_{2}^{6}>$

Notemos que neste caso, diferentemente de quando calculados os idempotentes primitivos de $\mathbb{F}_{2} G \mathbf{e}_{1}, \widehat{H}_{3}$ não comuta com $\widehat{H}_{2}$ em $\mathbb{F}_{2} G \mathbf{e}_{2}$. Todos os seis idempotentes calculados dão origem a códigos ótimos $[21,3,12]$ sobre $\mathbb{F}_{2}$.

\section{Exemplo 5.5(Construção de Código Ótimo $[32,2,24]$ em $\mathbb{F}_{3}$ )}

Em $\mathbb{F}_{2}$, considere $G=G_{[32,13]} \cong C_{8} \rtimes C_{4}$. Assim, temos $G$ descrito por

$$
G=<a, b \mid, a^{8}=b^{4}=1, b a b^{-1}=a^{3}>
$$

então temos em $\mathbb{F}_{3} G_{[32,13]}$ a seguinte estrututa

$$
\mathbb{F}_{2} G \cong 4 \mathbb{F}_{3} \oplus 2 \mathbb{F}_{9} \oplus 6 M_{2}\left(\mathbb{F}_{3}\right)
$$

Se tomarmos $\mathbf{e}=2+a^{-2}+b^{2}+2 a^{4}+2 a b a^{-1} b+a^{2}+(a b)^{2}+2 a^{2} b^{2}$ como idempotente primitivo central, e é tal que $\mathbb{F}_{3} G \cong M_{2}\left(\mathbb{F}_{3}\right)$, e executando-se $\operatorname{HatMapXi}(1, \mathbf{e}, \mathbb{F} G)$, obtemos $\mathscr{A}=[[[1],[[3,1],[2,2],[2,4]]], 1]_{\mathfrak{m i s t o}}$.

Dessa forma, em $\mathbb{F}_{3} G \mathbf{e}$, um par de idempotentes primitivos é dado por

$$
\begin{aligned}
e_{1} & =\xi\left(\left.\mathscr{A}\right|_{1}\right) \\
& =\xi([[1],[[3,1],[2,2],[2,4]]]) \\
& =(1-0) \widehat{H}_{1}(1-0)\left(2 g_{1}+g_{2}+g_{4}\right)(1-0) \widehat{H}_{1}(1-0) \mathbf{e} \\
& =\left(2 g_{1}+g_{2}+g_{4}\right) \mathbf{e} \\
e_{2} & =\left(1-e_{1}\right) \widehat{H}_{1}\left(1-e_{1}\right) \mathbf{e} \\
& =\left(1-e_{1}\right) \mathbf{e}
\end{aligned}
$$

Sendo $g_{1}=(1), g_{2}=a$ e $g_{4}=b$, o idempotente $e_{1}=(2+a+b)$ e fornece um Código Ótimo $[32,2,24]$ em $\mathbb{F}_{3}$.

\section{Exemplo 5.6(Construção de Código Ótimo $[20,4,12]$ em $\mathbb{F}_{3}$ )}

Seja $G=G_{[20,1]}$,com

$$
G_{[20,1]}=<a, b \mid, a^{5}=b^{4}=1 b a b^{-1}=a^{-1}>
$$


Ainda em $\mathbb{F}_{3}$, temos

$$
\mathbb{F}_{3} G \cong \mathbb{F}_{3} \oplus \mathbb{F}_{3} \oplus \mathbb{F}_{9} \oplus M_{2}\left(\mathbb{F}_{9}\right) \oplus M_{2}\left(\mathbb{F}_{9}\right)
$$

Sendo $\mathbf{e}=1+2 b^{2}+2 a+a b^{2}+2 a^{2}+a^{2} b^{2}+2 a^{3}+a^{3} b^{2}+2 a^{4}+a^{4} b^{2}$

Então, HatMapXi(1, e, $\mathbb{F} G)$ retorna $\mathscr{A}=[[[1],[[3,1],[3,2],[2,3],[3,4],[2,7]]], 1]_{\text {misto }}$

Ambos os idempotentes obtidos por $\xi\left(\left.\mathscr{A}\right|_{1}\right)$ e $\xi\left(\left.\mathscr{A}\right|_{2}\right)$ geram códigos ótimos $[20,4,12]$ em $\mathbb{F}_{3}$.

\section{Exemplo 5.7(Construção de Código Ótimo $[16,2,12]$ em $\mathbb{F}_{3}$ )}

Tememos $G=G_{[16,8]}$ com

$$
\begin{gathered}
G_{[16,8]}=<a, b \mid, a^{8}=b^{2}=1, b a b^{-1}=a^{3}> \\
\mathbb{F}_{3} G \cong 4 \mathbb{F}_{3} \oplus 3 M_{2}\left(\mathbb{F}_{3}\right)
\end{gathered}
$$

Tomando o idempotente central $e=1+2 a+2 a^{4}+a^{7}+a^{5}+2 a^{3}$, executandose HatMapXi $(1, \mathbf{e}, \mathbb{F} G)$, obtemos $\mathscr{A}=[3,1]$, ou seja, um conjunto completo de idempotentes primitivos de $\mathbb{F}_{3} G \mathbf{e}$ dados por $\mathscr{A}$ é

$$
\begin{aligned}
& e_{1}=\xi\left(\left.\mathscr{A}\right|_{1}\right)=\widehat{H}_{3} \mathbf{e} \\
& e_{2}=\xi(\mathscr{A})=\left(1-e_{1}\right) \mathbf{e}
\end{aligned}
$$

Onde $H_{3}=<b>$.

Ambos geram um Código Ótimo $[\mathbf{1 6 , 2 , 1 2}]$ em $\mathbb{F}_{3}$.

Diversos outros Códigos Ótimos podem ser construídos da mesma maneira, vale ressaltar que para os grupos fortemente monomiais é possível descrever seus idempotentes por meio de Pares de Shoda fortes. 


$\begin{array}{llllc}\text { Corpo } & \text { Grupo } & \text { Estrutura } & \text { Código } & \text { Tipo de Endereço } \\ \mathbb{F}_{2} & G_{[21,1]} & C_{7} \rtimes C_{3} & {[21,3,12]} & H \\ \mathbb{F}_{3} & G_{[32,13]} & C_{8} \rtimes C_{4} & {[32,2,24]} & M \\ \mathbb{F}_{3} & G_{[20,1]} & C_{5} \rtimes C_{4} & {[20,4,12]} & M \\ \mathbb{F}_{3} & G_{[16,8]} & Q D_{16} & {[16,2,12]} & H \\ \mathbb{F}_{3} & G_{[32,9]} & \left(C_{8} \rtimes C_{2}\right) \rtimes C_{2} & {[32,2,24]} & H \\ \mathbb{F}_{3} & G_{[16,12]} & C_{2} \rtimes Q_{8} & {[16,2,12]} & M \\ \mathbb{F}_{5} & G_{[24,1]} & C_{3} \rtimes C_{8} & {[24,2,20]} & M\end{array}$

\section{Exemplo 5.8 (Idempotentes Primitivos em $\mathbb{F}_{7} S_{5}$ e comparação com Pares de Shoda)}

Considere $\mathbb{F}_{7} S_{5}$. $S_{5}$ não é fortemente monomial.

De fato, seus únicos Pares Shoda Fortes são $\left(S_{5}, S_{5}\right)$ e $\left(S_{5}, A_{5}\right)$.

Temos que então que

$$
\mathbb{F}_{7} S_{5} \cong \mathbb{F}_{7} \oplus \mathbb{F}_{7} \oplus M_{4}\left(\mathbb{F}_{7}\right) \oplus M_{4}\left(\mathbb{F}_{7}\right) \oplus M_{5}\left(\mathbb{F}_{7}\right) \oplus M_{5}\left(\mathbb{F}_{7}\right) \oplus M_{6}\left(\mathbb{F}_{7}\right)
$$

Seus idempotentes centrais primitivos referentes à parte Abeliana, são dados por

$\mathbf{e}_{1}=\hat{S}_{5}$ e $\mathbf{e}_{2}=\left(1-\hat{S}_{5}\right) \hat{S}_{5}^{\prime}=\left(1-\hat{S}_{5}\right) \widehat{A}_{5}$, porém, nenhum destes nos interessa.

Considere os demais idempotentes na respectiva ordem de suas componentes de Wedderburn

$$
\begin{array}{ll}
\mathbf{e}_{3}, \operatorname{com} \operatorname{dim}_{\mathbb{F}} \mathbb{F}_{7} S_{5} \mathbf{e}_{3}=4^{2} & \mathbf{e}_{4}, \operatorname{com} \operatorname{dim}_{\mathbb{F}} \mathbb{F}_{7} S_{5} \mathbf{e}_{3}=4^{2} \\
\mathbf{e}_{5}, \operatorname{com} \operatorname{dim}_{\mathbb{F}} \mathbb{F}_{7} S_{5} \mathbf{e}_{3}=5^{2} & \mathbf{e}_{6}, \operatorname{com} \operatorname{dim}_{\mathbb{F}} \mathbb{F}_{7} S_{5} \mathbf{e}_{3}=5^{2} \\
\mathbf{e}_{7}, \operatorname{com} \operatorname{dim}_{\mathbb{F}} \mathbb{F}_{7} S_{5} \mathbf{e}_{3}=6^{2} &
\end{array}
$$

Obtidos através de PrimitiveCentralIdempotentsByCharacterTable

Por meio de HatMapXi aplicada em cada um dos idempotentes centrais primitivos $\mathbf{e}_{3}, \ldots, \mathbf{e}_{6}$, obtemos os seguintes endereços:

Temos que: 


$$
\begin{aligned}
& \mathscr{A}_{3}=[2,7,16,1] \\
& \mathscr{A}_{4}=[37,12,7,1] \\
& \mathscr{A}_{5}=[27,58,6,10,1] \\
& \mathscr{A}_{6}=[27,45,2,7,1] \\
& \mathscr{A}_{7}=[42,12,43,13,4,1]
\end{aligned}
$$

Então, $\mathscr{A}_{7}=[42,12,43,13,4,1]$ nos diz que com os grupos dados por:

$$
\begin{array}{ll}
\widehat{H}_{42}=<(1,2,4,3)> & \widehat{H}_{12}=<(1,4)(2,3)> \\
\widehat{H}_{43}=<(1,2,3,4)> & \widehat{H}_{13}=<(1,3)(2,4)> \\
\widehat{H}_{4}=<(3,4)>, & \widehat{H}_{1}=<I d_{S_{5}}>
\end{array}
$$

podemos determinar um conjunto completo de idempotentes primitivos de $\mathbb{F}_{7} S_{5} \mathbf{e}_{7}$ . Como todos estes grupos comutam 2 a 2, seus idempotentes são dados dado por

$$
\begin{aligned}
e_{1} & =\widehat{H}_{42} \mathbf{e}_{7} \\
e_{2} & =\widehat{H}_{12}\left(1-\hat{s}_{42}\right) \mathbf{e}_{7} \\
e_{3} & =\widehat{H}_{43}\left(1-e_{1}-e_{2}\right) \mathbf{e}_{7} \\
e_{4} & =\widehat{H}_{13}\left(1-e_{1}-e_{2}-e_{3}\right) \mathbf{e}_{7} \\
e_{5} & =\widehat{H}_{4}\left(1-e_{1}-e_{2}-e_{3}-e_{4}\right) \mathbf{e}_{7} \\
e_{6} & =\widehat{H}_{1}\left(1-e_{1}-e_{2}-e_{3}-e_{4}-e_{5}\right) \mathbf{e}_{7}
\end{aligned}
$$

são idempotentes primitivos de $\mathbb{F}_{7} S_{5} \mathbf{e}_{7}$.

Utilizando-se qualquer um destes idempotentes, podemos construir um código linear sobre $\mathbb{F}_{7}$ com os parâmetros $[120,6,40]$.

\section{Exemplo 5.9(Idempotentes Primitivos em $\mathbb{F}_{7} S_{6}$ )}

Mesmo grupos com ordens maiores podem possuir endereços puramente $\mathrm{Hat}$, ou seja do tipo $\mathfrak{h}$. Pode-se observar isso tanto no exemplo anterior $\left(\mathbb{F}_{7} S_{5}\right)$, bem como o será em $\mathbb{F}_{7} S_{6}$.

Para $\mathbb{F}_{7} S_{6}$ temos a seguinte decomposição de Wedderburn: 


$$
\mathbb{F}_{7} S_{6} \cong 2 \mathbb{F}_{7} \oplus 4 M_{5}\left(\mathbb{F}_{7}\right) \oplus 2 M_{9}\left(\mathbb{F}_{5}\right) \oplus 2 M_{10}\left(\mathbb{F}_{5}\right) \oplus M_{16}(\mathbb{F})
$$

Ambas as componentes $M_{10}\left(\mathbb{F}_{5}\right)$ possuem idempotentes primitivos descritos por

$$
\mathscr{A}=[233,235,237,291,383,117,393,3,13,1]
$$

Onde:

$$
\begin{array}{ll}
H_{233}=<(2,6)(3,4),(1,5)>, & H_{235}=<(3,5)(4,6),(1,2)>, \\
H_{237}=<(1,2,3,4)>, & H_{291}=<(1,3)(4,5),(4,5)>, \\
H_{383}=<(2,5,4,3,6)>, & H_{117}=<(1,2)(5,6),(3,4)(5,6)>, \\
H_{393}=<(1,6,2,4,5)>, & H_{3}=<(4,6)>, \\
H_{13}=<(1,5)>, & H_{1}=<I d_{S_{5}}>
\end{array}
$$

Ou seja, por meio de $\xi\left(\left.\mathscr{A}\right|_{i}\right), 1 \leq i \leq 10$, obtemos um conjunto completo de idempotentes primitivos em qualquer uma das componentes $\mathbb{F}_{7} S_{6} \mathbf{e} \cong M_{10}\left(\mathbb{F}_{5}\right)$. 


\section{Capítulo 6}

\section{Demais Algoritmos em Gap}

Nesta parte final, deixaremos listadas as principais funções utilizadas, mesmo que não citadas explicitamente. Algumas delas possuem função complementar a outras funções e não possuem significado sozinhas. Em seguida, deixamos o código dos principais algoritmos não apresentados durante os capítulos principais.

\subsection{Lista De Funções}

Esta lista corresponde à lista de funções e algoritmos produzidos, isto é, não presentes atualmente no GAP ou em outro pacote.

BaseofeFGe (FG, e):

Usa escalonamento em $\mathbb{F}$ para determinar uma base de e $\mathbb{F} G$ (bilateral).

BaseofFGe (FG, e):

Usa escalonamento em $\mathbb{F}$ para determinar uma base de $\mathbb{F} G e$.

$\mathbf{C}\left(e_{1}, e_{2}, \mathbf{F G}\right)$ :

Função de Competição.

Challenge (E, FG):

Aplicação da Função de competição .

CoeficientesByG (FG, G, x):

Retorna os coeficientes de $x$ na mesma ordem de List $(G)$.

DimHate (FG, e , s):

Lista dimensões de todos os produtos s $\mathbb{F} G$ se. 
DimHate2 (FG, e, $\operatorname{dim} . .$.$) :$

DimHate em um único elemento.

DuploCrivo (dima , dim, prod):

Retorna 1 se as dimensões são iguais e prod é idempotente, retorna 2 se prod é idempotente mas dimensões são distintas e retorna 3 se prod não é idempotente.

EV5 (ParametroDeSaida, ListaDeParametros):

Engine de $\Xi$, ou seja, HatMapXI.

$\operatorname{Dim}($ FG, e):

Verificador de dimensão para e $\mathbb{F} G e$.

FiltroCentralizeXemL (x, L, FG):

Recebe $X$ e filtra quem comuta com os elementos de uma lista $L$.

FiltroIdempotent ( L, FG):

Zera os elementos não idempotentes.

ForceMidp (FG, listaG, eforce, dim, m, dimmin):

Retorna um endereço misto.

Hat (H, FG):

Recebe $H$ devolve $\widehat{H}$.

HatMap (e,s,FG,nivel,versao):

Faz uma busca pelo HatMap.

HatMapXi (n,e,s,FG,Sk):

Retorna um endereço sem fazer o mapa completo função $\Xi$.

Hats (Subgrupos, FG):

Retorna um chapéu para cada subgrupo.

Hunter (c,tc):

Gerencia tarefas por diversos núcleos.

\section{HunterEngine:}

Mecanismo de busca por raízes de polinômios com coeficientes em $\mathbb{F} G$.

IdByHatAdressSum ( lista, e, hats, FG):

Recebe endereço, Retorna idempotentes (função $\xi$ ). 
IdByHatMix (A,neg,e,FG):

Função $\xi$ para endereços mistos.

IdempMHF (ListaDeParametros):

Função Interna do Algoritmo $\Xi$ completo, retorna idempotentes por endereços mistos.

Lista2Base (FG, G, Lista):

Reduz uma lista de geradores a uma $\mathbb{F}$-base.

MatrixBase (listaidemps, FG):

MatrixBase Retorna uma matriz cujas entradas são uma $\mathbb{F}$-base para $F p^{1}$ (corpo de decomposição).

\section{MatrixByIdemps (listaidemps,FG):}

Recebe uma Lista de Idempotentes e retorna $E_{i j}$ tal que $E_{i i}=e_{i}$.

MatrixCorrector (Mat,FG):

Realiza operações, retona $E_{i j}$, ou false caso contrário.

MatrixTester (M,FG):

Testes de verificação matricial.

minpotidemp (a, FG)

Retorna a minima potencia que torna a idempotente.

MultiHatsSS (FG, e,dimmin, iterac,m):

Multi Hat

npot (a, FG)

Recebe um representante a da soma de classes y e retorna $n$ tal que $y^{n} e^{\prime}$ idempotente.

\section{OneMatCorrection (Mat,FG,NmexerLista):}

Executa uma única correção com intúito de criar uma matriz Pivot.

\section{PCIbySOCI (FG):}

Retorna Idempotentes Centrais Primitivos. Determina um conjunto de centrais contendo os primitivos e aplica Challenge.

prods (lista, FG):

Recebe uma lista e retorna os termos do produto de cisão (a+(1-a) ) $(b+(1-b)) \ldots$ 
ProgGbase (FG, listaG, e, dim):

Retorna uma base e uma base multiplicando-se por um idempotente.

ProgTest ( FG, e, dim... ):

Função de Dimensão.

ProgTestT ( FG, e, H, dim... ):

Função de Dimensão, realiza teste em um transversal de $H$ em $G$.

Separa (FG):

Recebe $F G$ retorna o par $[F, G]$.

ShellMaker (s, C, e, FG, parametro):

Elaboração de Camadas na função de redução de dimensão.

SOCI (FG):

Some Ortogonal Central Idempotents (FG).

$\operatorname{StSk}(\mathbf{n})$ :

Função de Salto Padrão, Standart Skip $-s_{k}(n)=(n, 1,1, . ., 1)$.

SubSum (H, FG):

Soma de elementos de um subconjunto de FG.

TestIdempFGe (lista, e, FG, num...):

Testa se uma lista de elementos corresponde a idempotentes ortogonais de FGe.

Vetorize2GR (n, FG, base):

Recebe um número retorna um elemento de $F G$.

Vetorize2multibase (n, Mbase):

Rece um natural, retorna sua escrita em uma base não regular.

Vetorize2mupla (n, base,m):

Recebe $n$ e retorna escrita de $n$ em uma base.

Y (FG):

Função soma de classes.

Os presentes algoritmos são em sua grande maioria implementação direta das construções apresentadas. Entretanto, algumas funções apresentam certa variação quanto ao nome ou método de entrada. Vale ressaltar que pequenas variações no 
código fonte levam a uma eficiência maior para determinados tipos de grupos.

Por tal motivo, as funções $\zeta, \xi$ e $\Omega$ serão dependentes de uma escolha de um mecanismo padronizado, os engines.

\subsection{Algumas Funções Importantes}

Deixamos por fim, explicitamente os algoritmos que julgamos mais importantes para o funcionamento das funções principais.

BaseofeFGe: Usa escalonamento em $\mathbb{F}$ para determinar uma base de $\mathbb{F} G e$. Necessita da função CoeficientesByG.

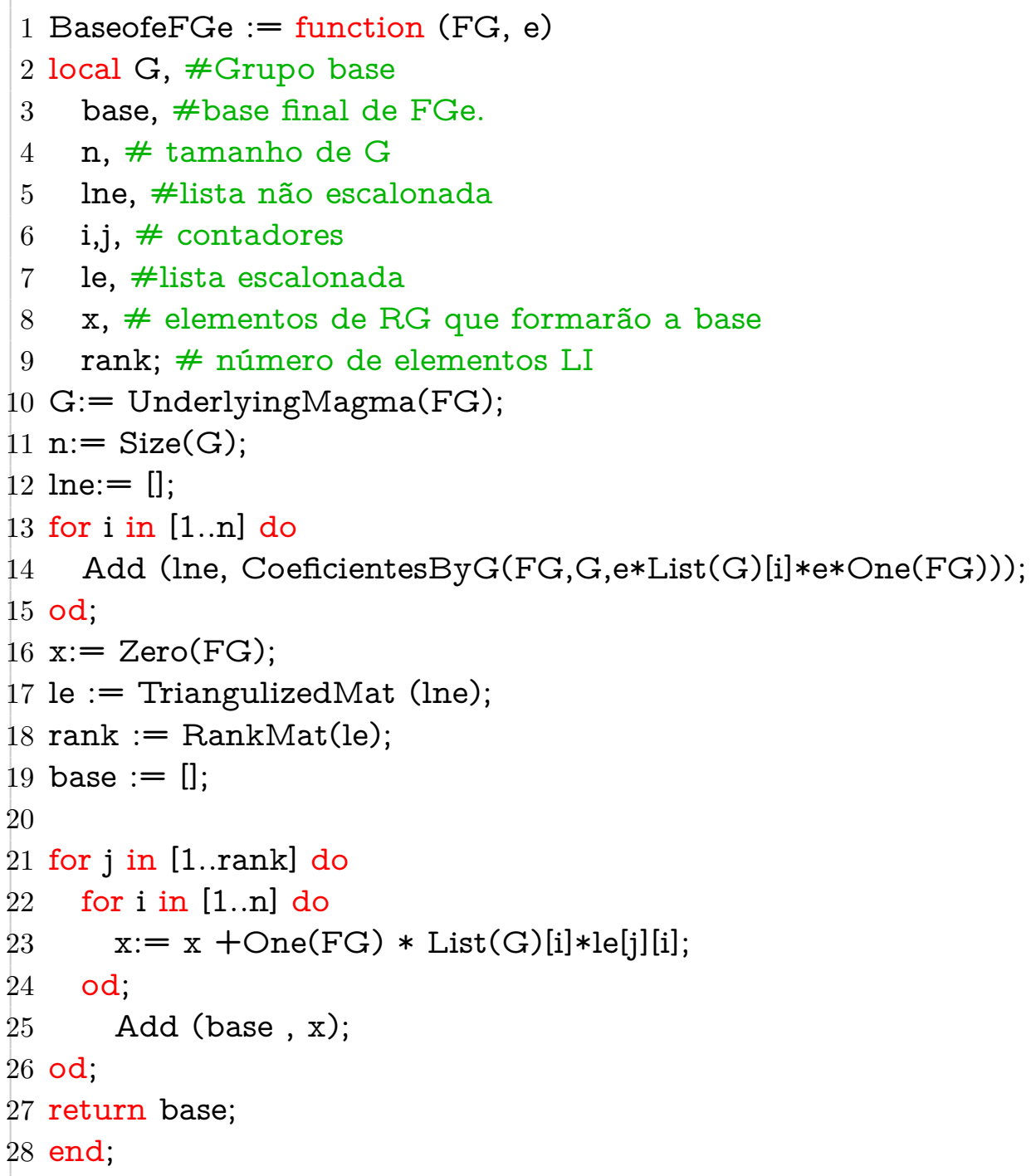


DuploCrivo: Função de crivo que exerce papel no algoritmo $\Xi$. Recebe duas dimensões e um elemento prod. Retorna 1 se as dimensões são iguais e prod é idempotente, retorna 2 se prod é idempotente mas dimensões são distintas e retorna 3 se prod não é idempotente

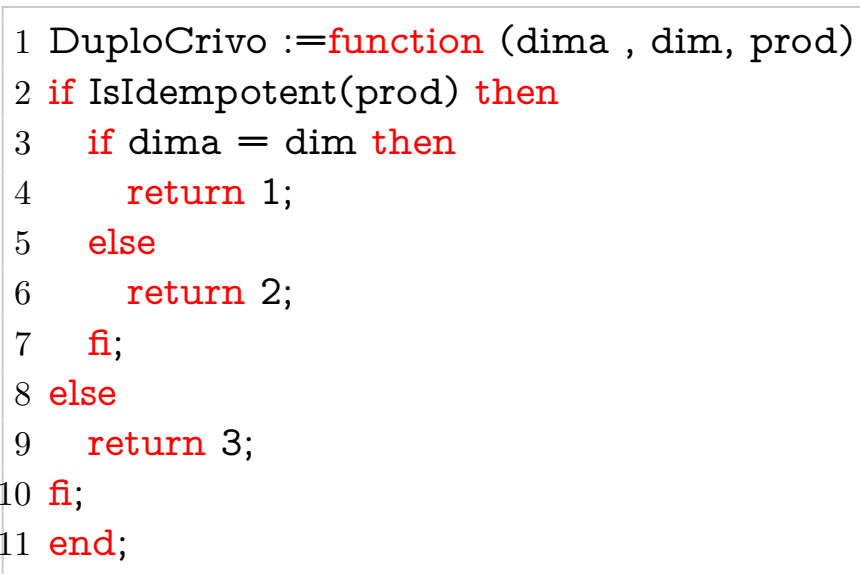

ForceMIdp:Retorna o idempotente e sua escrita em base limpa respectivamente, retornando uma 5-úpla [idemp ,idemp limpo, coord,baselimpa, Map] com o idempotente escrito de diferentes formas. Mantém informações e instruções sobre como reconstruir o idempotente.

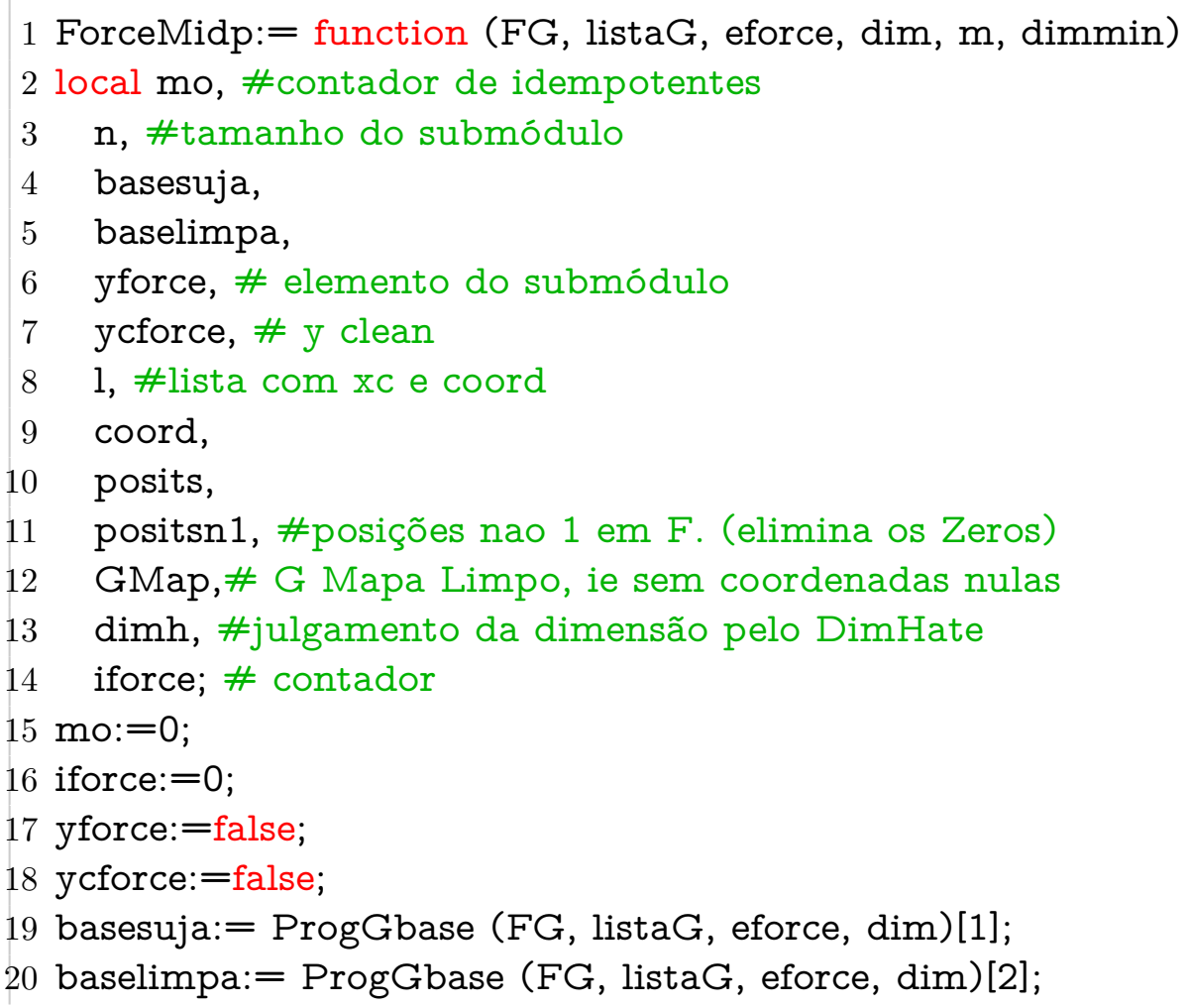


$21 \mathrm{n}:=\operatorname{Size}($ LeftActingDomain(FG))^Size(basesuja);

22 while $\mathrm{mo}<\mathrm{m}$ and iforce $<=\mathrm{n}$ do \#n pois a unidade foi descartada e será reposta no final.

23 iforce:=iforce+1;

24 yforce:= Vetorize2Fbase (iforce, FG,basesuja)[1];

25 if (yforce ${ }^{-} 2=y$ force) and (yforce $<>\operatorname{Zero}(F G)$ ) then

26 \#\#\# Julgar a dimensao.

27 dimh:= DimHate (FG, yforce, $[$ One $(F G)])[1] ;$

28 if dimh $=$ dimmin then

$29 \mathrm{mo}:=\mathrm{mo}+1$

$30 \quad$ fi;

31 fi;

32 od;

33

34 if $\mathrm{mo}=\mathrm{m}$ then

$35 \mathrm{l}$ :=Vetorize2Fbase (iforce, FG, baselimpa);

36 ycforce: $=1[1]$;

37 coord: $=1[2]$

38 fi;

39 posits:= List(baselimpa, $\mathrm{x}$->Position (listaG, $\mathrm{x}$ ));

40

41 positsn $1:=$ Positions (List (coord, $x->x<>1$ ), true);

42 Map:= List (positsn1, $\mathrm{x}->$ [ $\operatorname{coord}[\mathrm{x}], \operatorname{posits}[\mathrm{x}]$ ] );

43

44 return [yforce,ycforce,coord, baselimpa, Map];

45 end;

Hat e Hats:

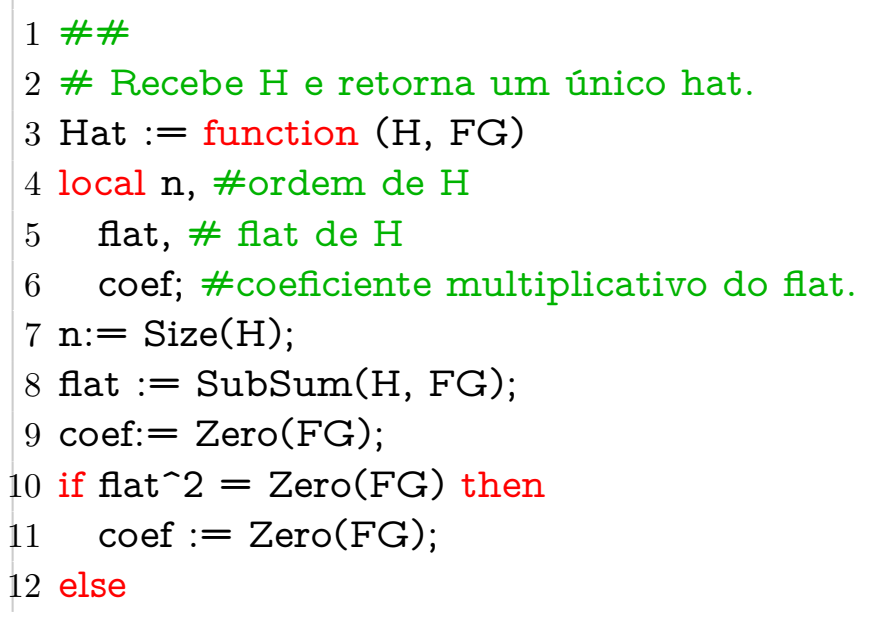




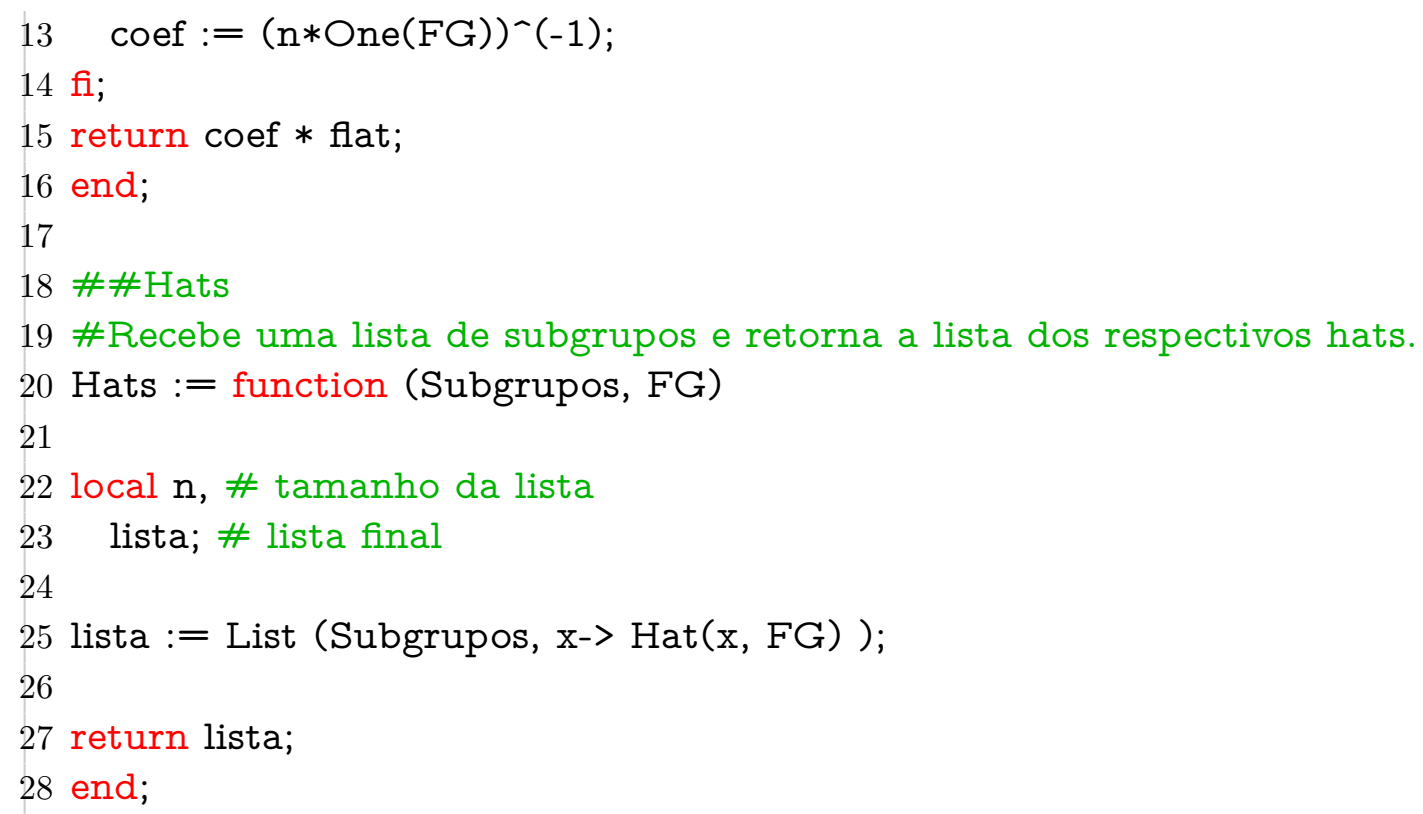

Função $\xi$ : Dada um Hat Adress por Soma, retorna os idempotentes correspondentes a esse endereço

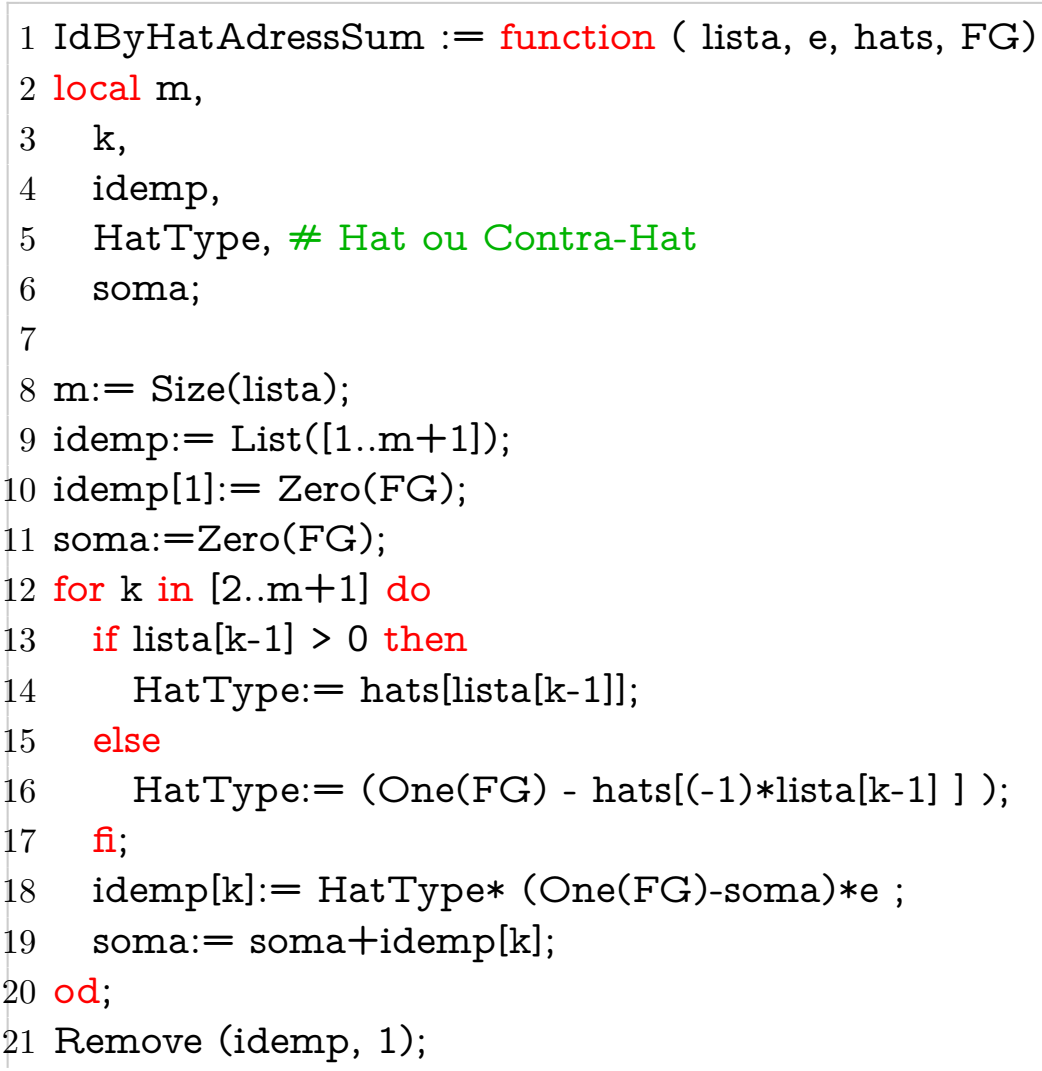


22 return idemp;

23 end;

Função $\xi$ para endereço misto: Recebe um endereço A, e o idempotente primitivo central e $n e g=\left(1-e_{1}-e_{2}-\ldots-e_{n}\right)$. Retorna o correspondente idempotente.

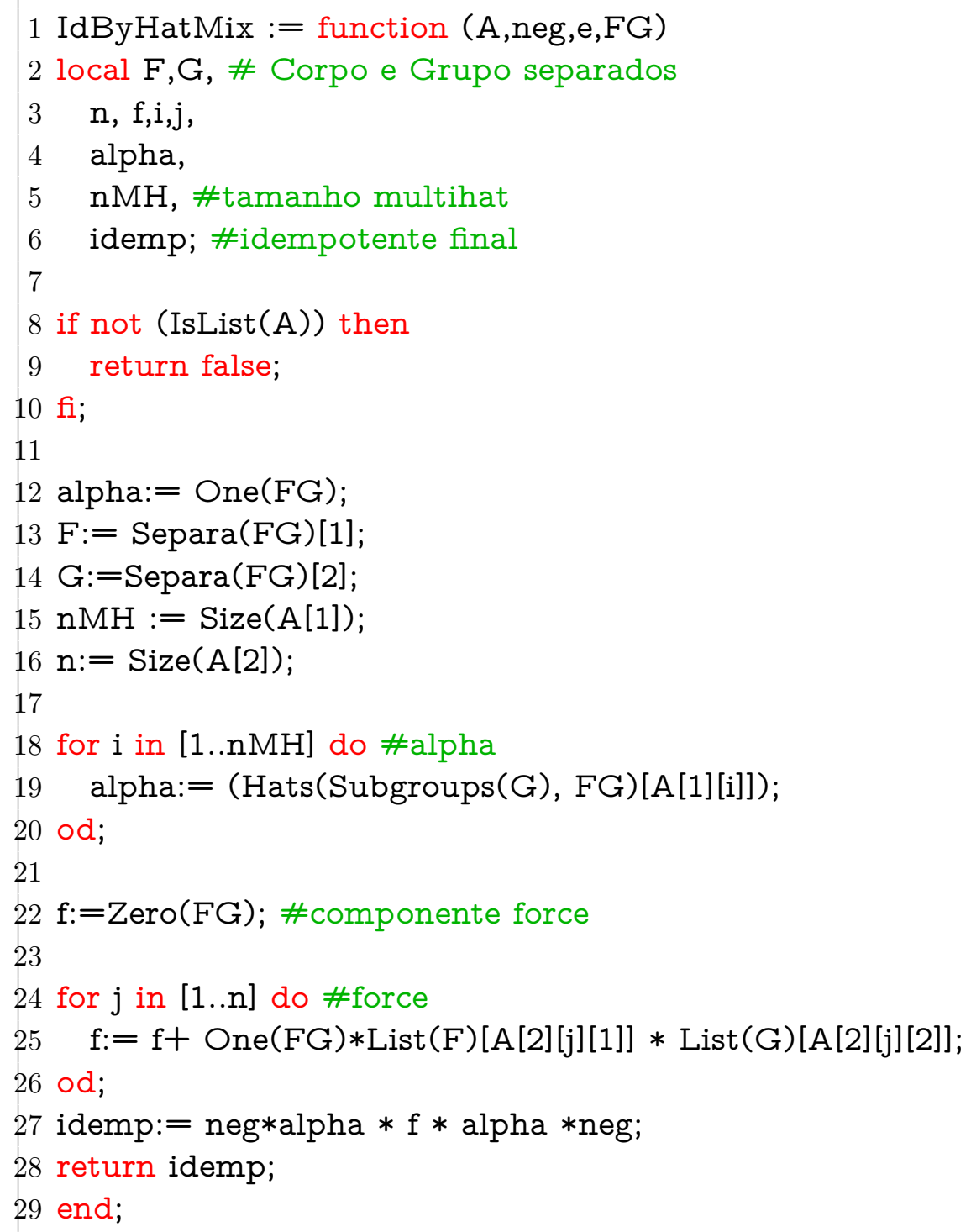

IdempMHf: Encontra idempotentes de endereço misto.

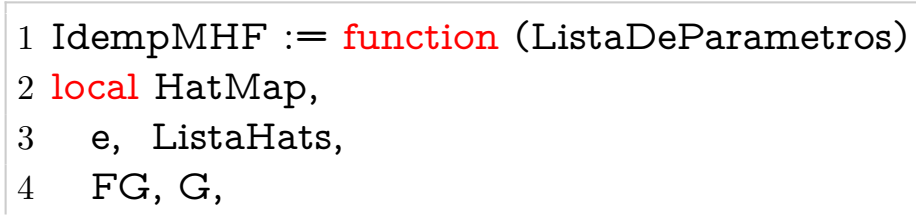




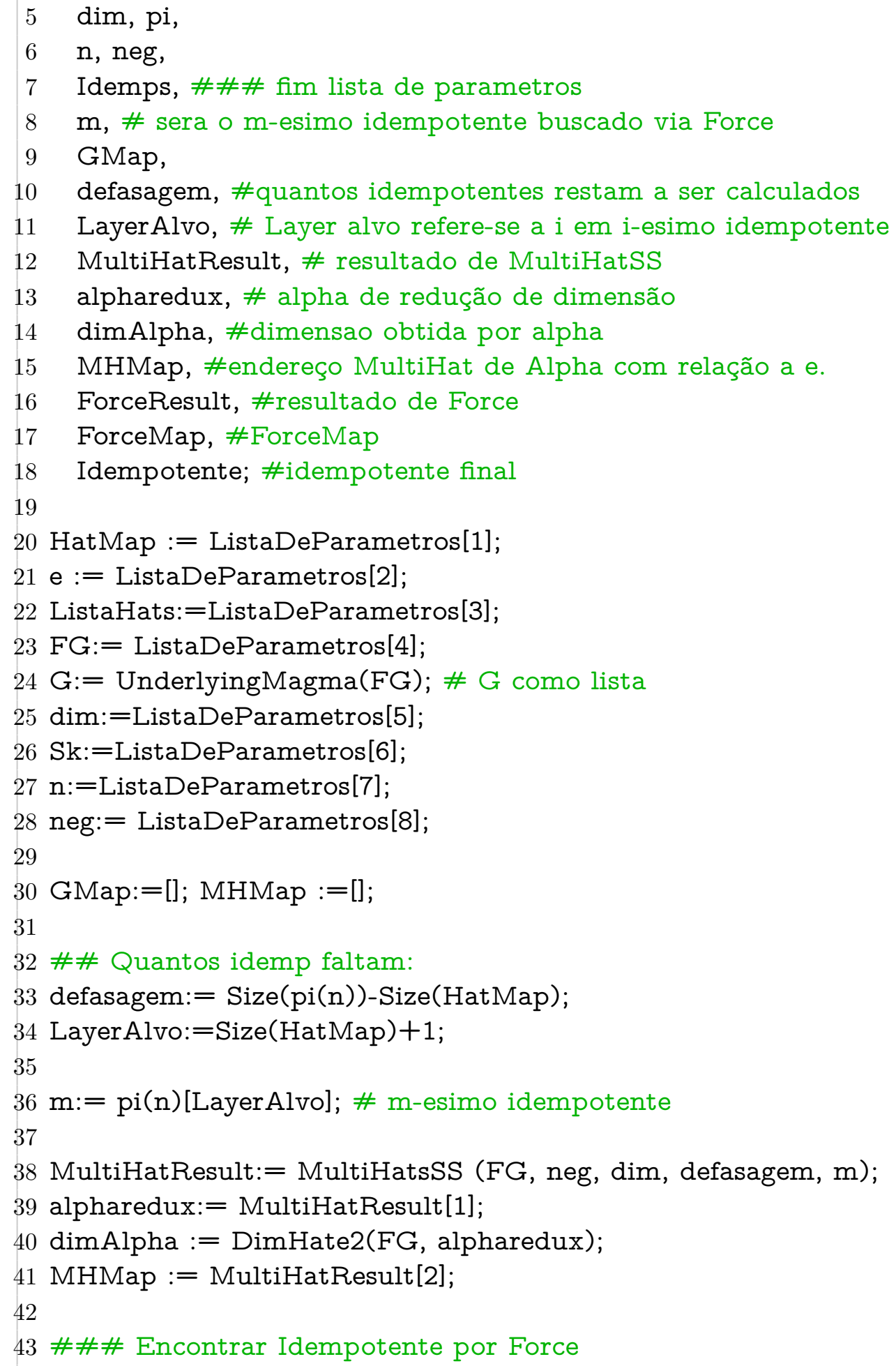


45 ForceResult := ForceMidp (FG, List(G), alpharedux, dimAlpha, m, dim );

46 ForceMap := ForceResult[5];

47 Idempotente:= ForceResult[1];

48

49 return [Idempotente, ,MHMap, ForceMap];

50 end;

Base Progressiva: Dada uma LISTA de elementos de $G$ (em geral o $G$ inteiro) e dado um idempotente e retorna um par [base suja, base limpa] onde base suja é a base para eFGe utilizando elementos do tipo e.LIST A.e e base limpa são os elementos da LIST A que formam essa base. Entra-se com a dimensão para agilizar cálculos.

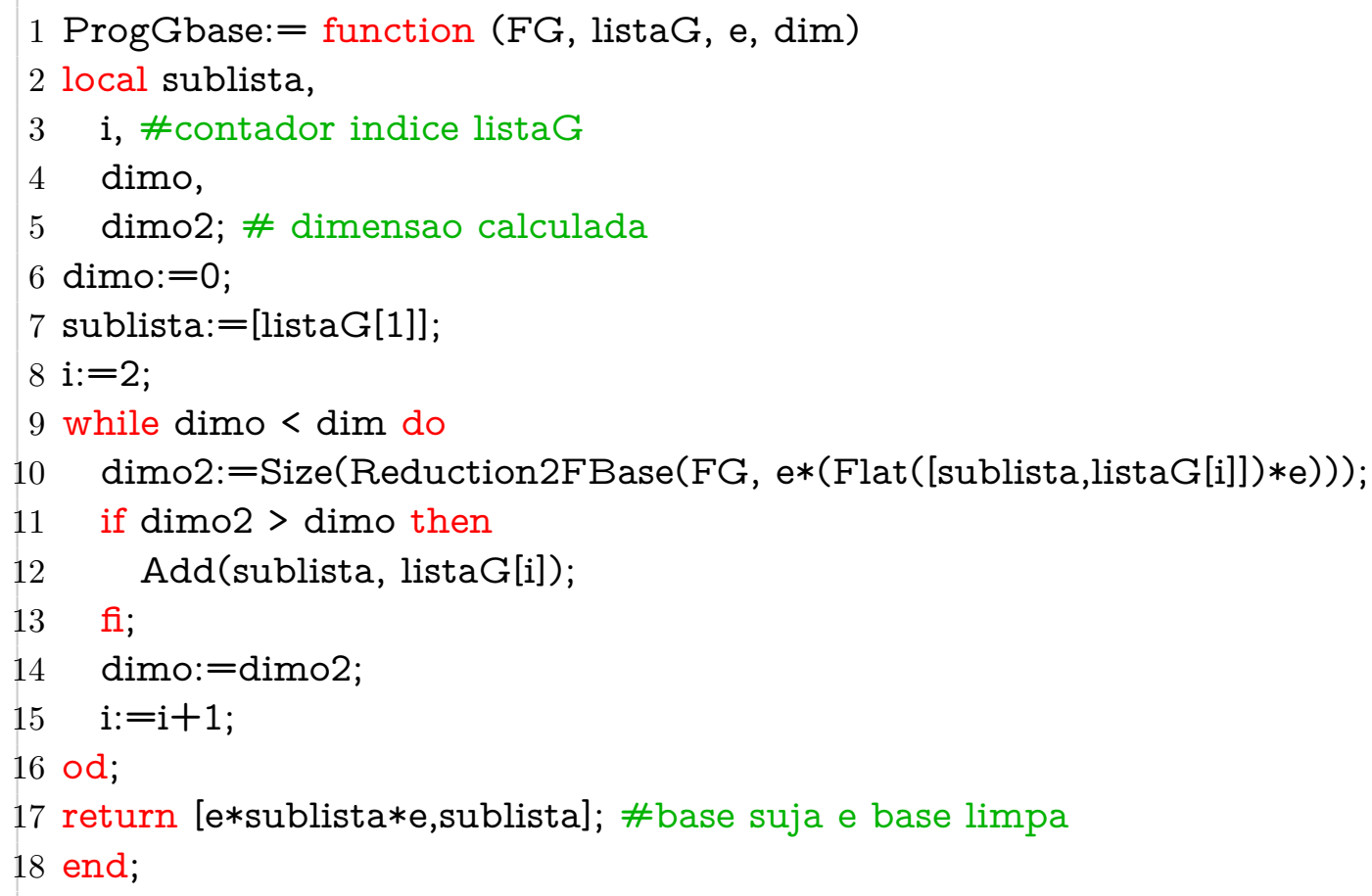

ShellMaker - Utilizada no algoritmo MultiHat

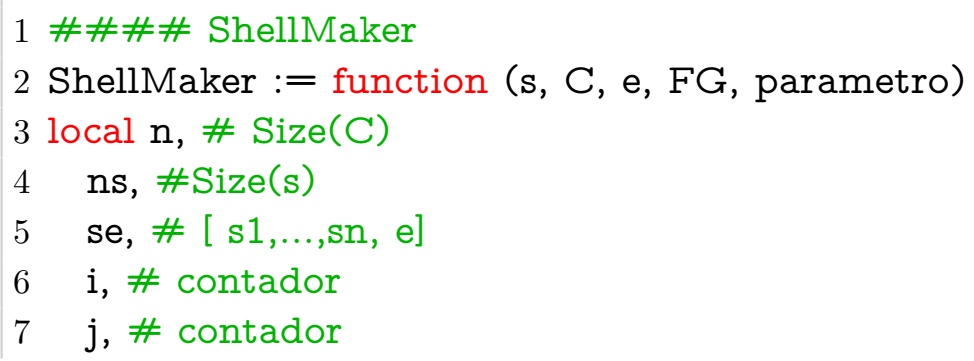


$8 \mathrm{k}$, \#contador

9 lista, \#lista anterior

10 list1,

11 list2,

$12 \quad \mathrm{IE}$, \#lista esquerda

$13 \operatorname{lrE}$, \# lista a remover esquerda

$14 \operatorname{lrD}$,\#lista a remover direita

15 ID, \#lista direita

16 lr;\#lista de remoção lr $=$ [1E, lD] Lista à direita e à esquerda

17

$18 \mathrm{n}:=\operatorname{Size}(\mathrm{C})$;

$19 \mathrm{~ns}:=\operatorname{Size}(\mathrm{s})$;

20 se := Flat ([s,e]);; \#adiciona o idempotente na ultima coordenada.

21 \# captura os que são não nulos

22 \# Criação da camada trivial (idemp) e primeira camada

23 if $\mathbf{C}=[]$ then

24 list $1:=[1 . . \mathrm{ns}]$;

25 if parametro $=-1$ then

$26 \mathrm{lD}:=$ Positions (List (list1, $\mathrm{x}->(\mathrm{s}[\mathrm{x}] * \mathrm{e}<>\operatorname{Zero}(\mathrm{FG})$ and $(\mathrm{s}[\mathrm{x}] * \mathrm{e}<>\mathrm{e})$

)) , true);

27 return [Flat([1,lD]), [ns+1]];

28 else

$29 \mathrm{lE}:=$ Positions (List (list1, $\mathrm{x}->(\mathrm{e} * \mathrm{~s}[\mathrm{x}]<>\operatorname{Zero}(\mathrm{FG})$ and $(\mathrm{s}[\mathrm{x}] * \mathrm{e}<>\mathrm{e}))$ ), true );

30 return $[[n s+1], F l a t([1,1 E])]$;

31 fi;

$32 \mathrm{fi} ;$

33

34 \# Abre listas para receberem elementos

$35 \mathrm{lD}:=[] ; \mathrm{lE}:=[] ; \operatorname{lrD}:=[] ; \operatorname{lrE}:=[] ;$ lista:=List $([1 . . \mathrm{ns}])$;

36

37 \#\# Demais camadas

38 if parametro $=1$ then

39 for $\mathrm{j}$ in [2..n] do

40 for $i$ in $[j . . n]$ do

41 list $1:=\operatorname{ComutSL}(\mathrm{s}$, List $(\mathrm{C}[\mathrm{i}], \mathrm{x}->\mathrm{s}[\mathrm{x}]))$;

42 list2:= Intersection (list1, lista);

43 lista:=list2;

44 od; \#\#\# terminaram as intersecções

45 for $\mathrm{k}$ in [1..Size(lista)] do 
6 if $\operatorname{Set}(\operatorname{List}(\mathrm{C}[\mathrm{j}-1], \mathrm{x}->\mathrm{se}[\mathrm{x}]) * \operatorname{se}[$ lista[k]] $)=[$ Zero(FG)] then

Add (lrE, lista[k]); \# Retira camadas que anulam a anterior

$48 \quad$ fi;

49 if $\operatorname{Set}\left(\operatorname{List}\left(C[j-1], x_{-}>\operatorname{se}[x]\right) * \operatorname{se}[l i s t a[k]]\right)=\operatorname{Set}\left(\operatorname{List}\left(C[j-1], x^{->} \operatorname{se}[\right.\right.$ $\mathrm{x}])$ ) then

$50 \quad$ Add (lrE, lista[k]); \# Retira camadas que estabilizam a anterior $51 \quad$ fi;

52 od;

53 od;

54 else \# caso -1

55 for $\mathrm{j}$ in $[1 . . \mathrm{n}-1] \mathrm{do}$

56 for $i$ in $[1 . . j]$ do

57 list $1:=$ ComutSL ( $\mathrm{s}$, List (C $[\mathrm{i}], \mathrm{x}->\mathrm{s}[\mathrm{x}]$ )); \#atenção aqui $\mathrm{n}+1-\mathrm{i}$

58 list2:= Intersection (list1, lista);

59 lista:=list2;

60 od; \#\#\# terminaram as intersecções

61 for $\mathrm{k}$ in [1...Size(lista)] do

62 if $\operatorname{Set}(\operatorname{se}[\operatorname{lista}[\mathrm{k}]] * \operatorname{List}(\mathrm{C}[\mathrm{j}+1], \mathrm{x}->\operatorname{se}[\mathrm{x}]))=[\operatorname{Zero}(F G)]$ then

Add (lrD, lista[k]); \# Retira camadas que anulam a anterior fi;

if $\operatorname{Set}(\operatorname{se}[\operatorname{lista}[k]] * \operatorname{List}(C[j+1], x->\operatorname{se}[x]))=\operatorname{Set}(\operatorname{List}(C[j+1], x->$ $\mathrm{se}[\mathrm{x}])$ ) then

Add (lrD, lista[k]); \# Retira camadas que estabilizam a anterior

$67 \quad$ fi;

68 od;

69 od;

70 fi;

71

$72 \mathrm{lE}:=$ List $([1 . . \mathrm{ns}]) ;$

$73 \mathrm{lD}:=$ List $([1 . . \mathrm{ns}]) ;$;

74 SubtractSet (lE, Flat(lrE));

75 SubtractSet (ID, Flat(lrD));

76

77 if parametro $=1$ then \#controle de opção de saída

78 return $1 E$;

79 else

80 return $1 D$;

81 fi;

82 end; 
SOCI - Some Ortogonal Central Idempotents.

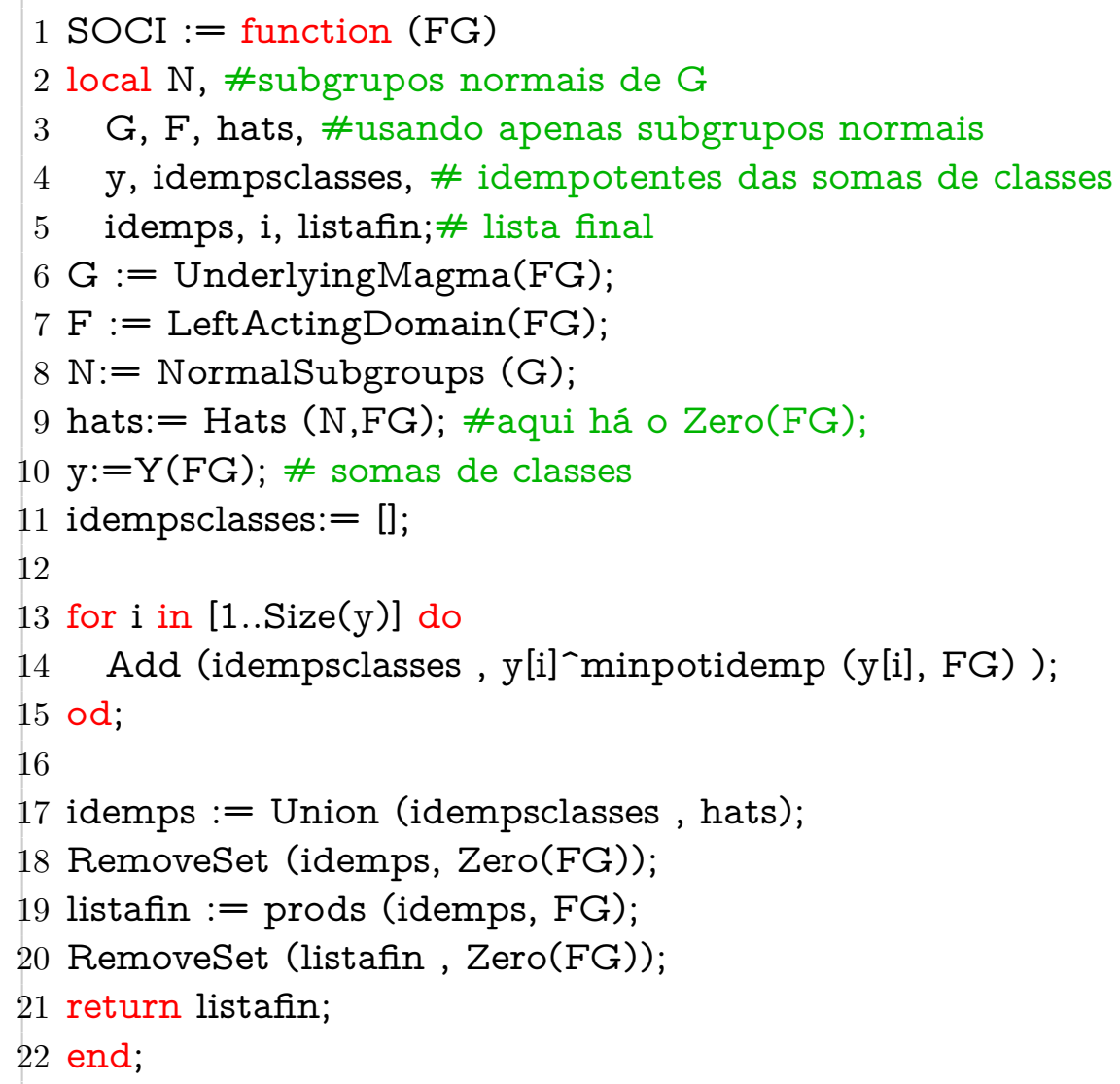

Função de salto Padrão: $S_{k}$. StSk - Standart Skip. Construtura de Skip Functions. Recebe n, número de idempotentes esperados. Retorna uma função $f(x)=$ $[x, 1, . ., 1]$ com $n$ entradas

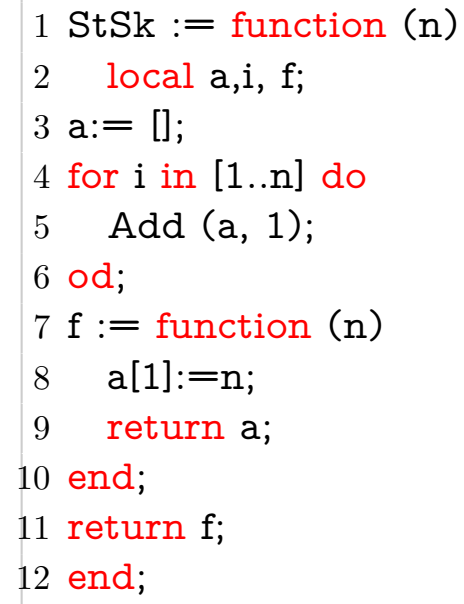


Vetorizador para Multibase: Dado um número e uma base, retorna esse número na base com $m$ coordenadas. Mbase deve ser uma lista contendo os tamanhos variados. O resultado deve sair na ordem base orientada à esquerda.

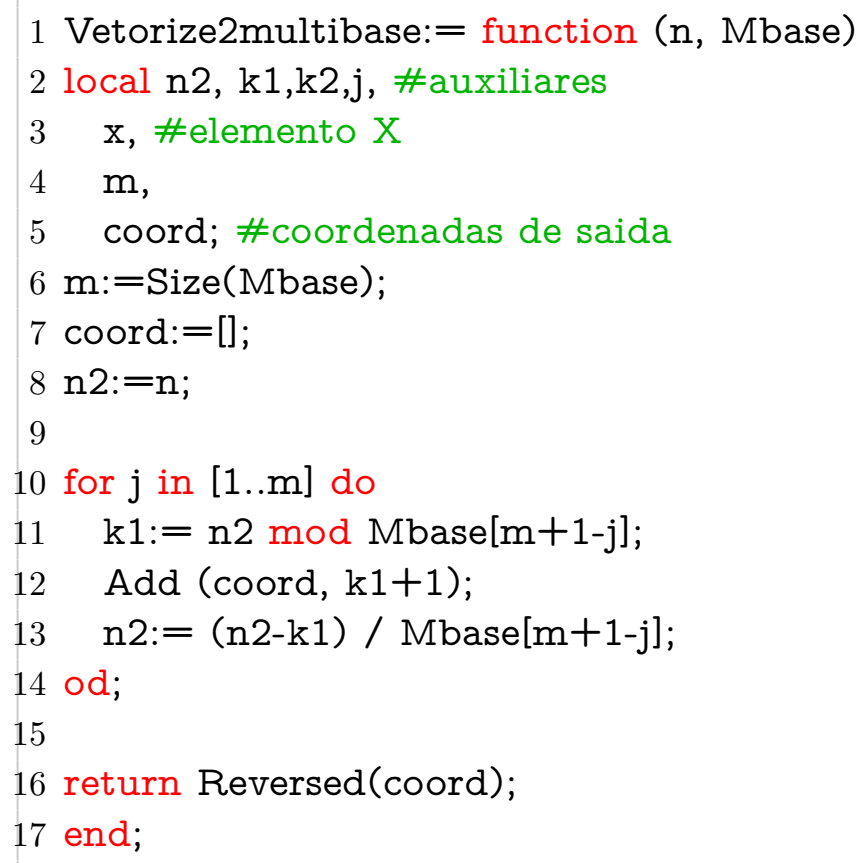

HunterEngine: Algoritmo poderoso de busca por raízes de um dado polinômio com coeficientes em $\mathbb{F} G$. Otimizado para trabalhar em paralelo. Variáveis são respectivamente:

númerodocore atual / total de cores / Opção de exibição /bound /FG / \# Polinômio / Detalhamento (0,1). / ExporType Tipo de resultado.

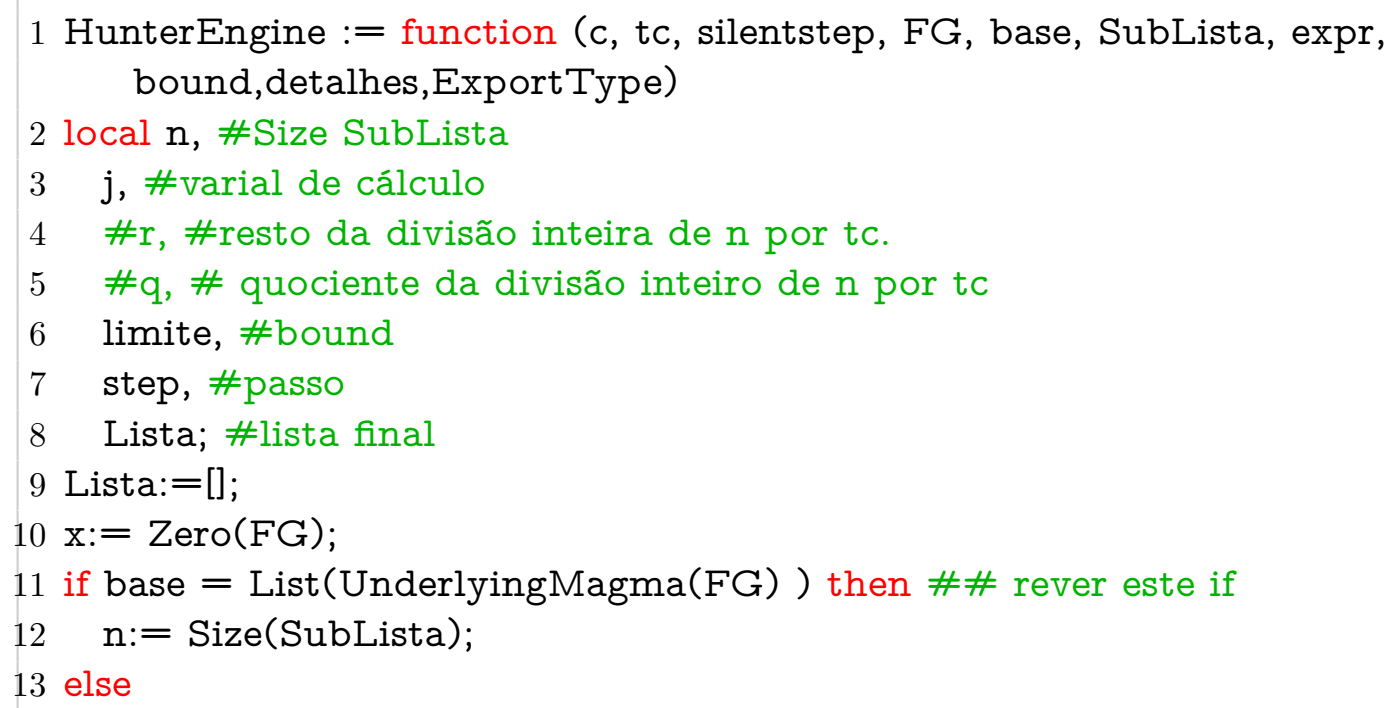




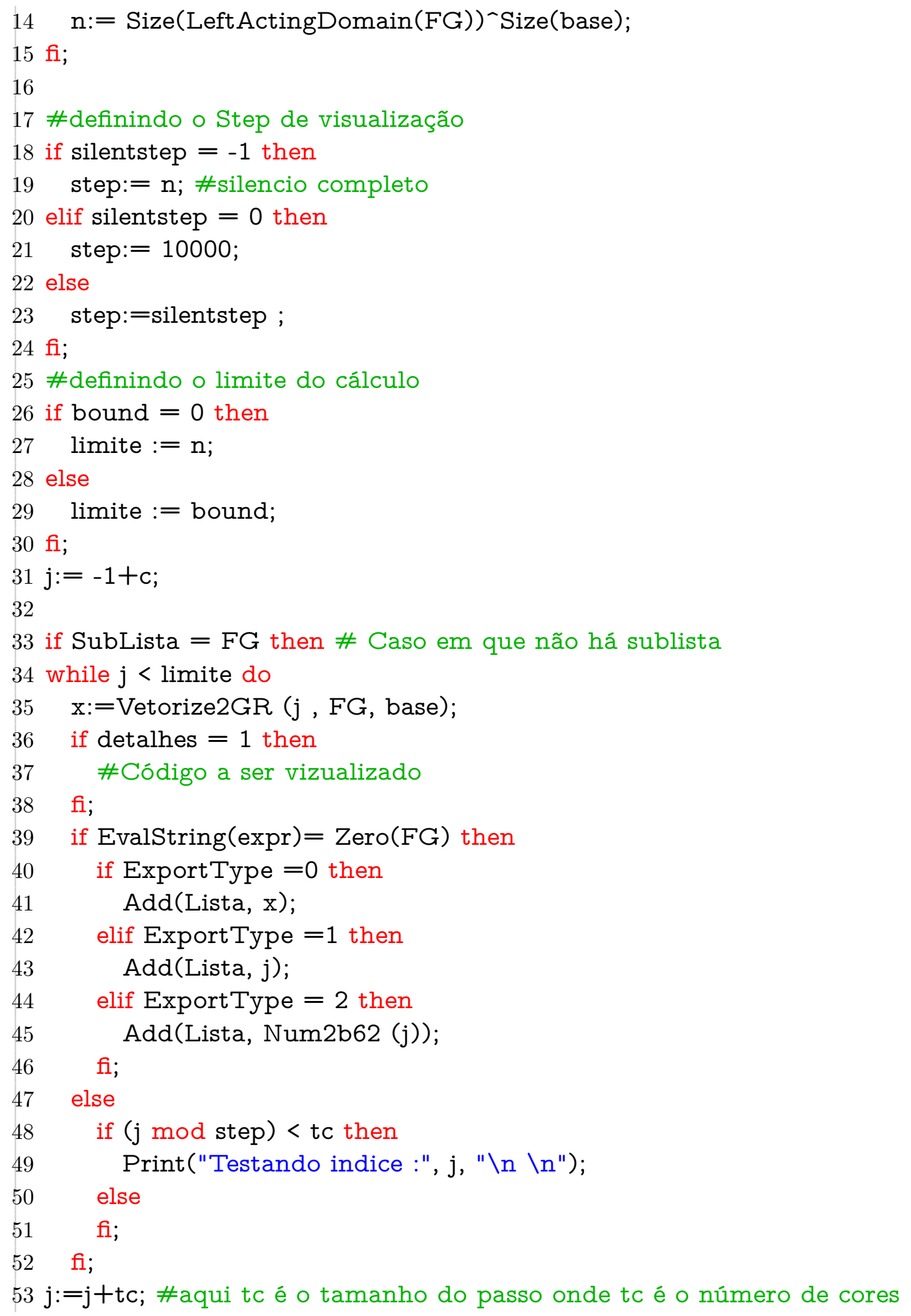




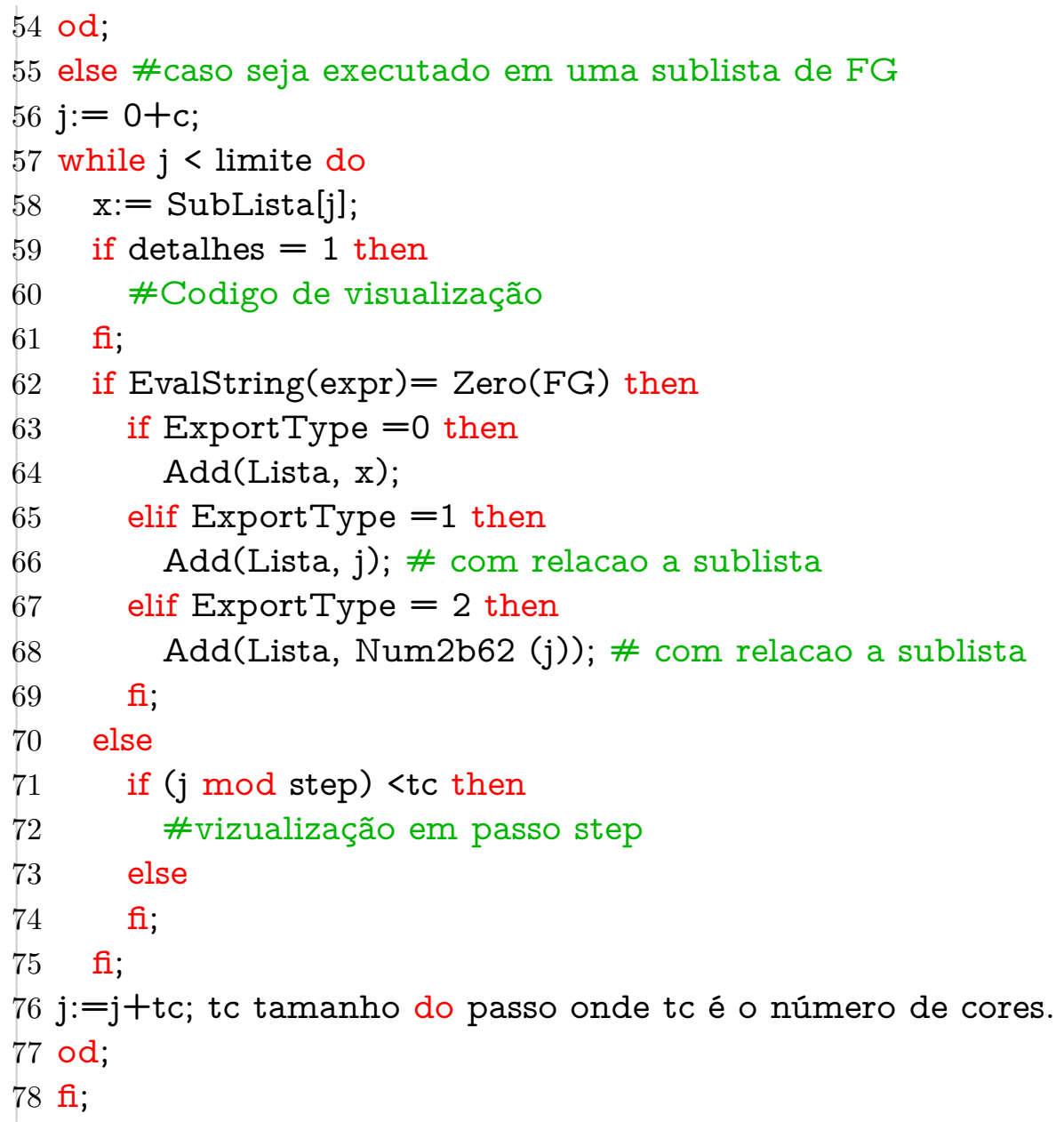




\section{Referências Bibliográficas}

[1] Baart R., Boothby T., Cramwinckel J., Fields J., Joyner D., Miller R., Minkes E., Roijackers E., Ruscio L., Tjhai C.: GUAVA-a GAP package.Version 3.12 (21/05/2012).http://www.gap-system.org/Packages/ guava.html.

[2] Bakshi, G. K. and Maheshwary, S. Extremely strong Shoda pairs with GAP - arXiv e-prints, 2015, arXiv:1503.05674

[3] Broche O., del Río Á.: Wedderburn decomposition of finite group algebras. Finite Fields Appl. 13(1), 71-79 (2007).

[4] Broche O., Herman A., Konovalov A., Olivieri A., Olteanu G., del Río Á., Van Gelder I.: Wedderga- Wedderburn Decomposition of Group Algebras. Version 4.6.0 (2013). http://www.cs.st-andrews.ac.uk/alexk/wedderga, http://www.gap-system.org/Packages/wedderga.html.

[5] The GAP Group: GAPGroups, Algorithms, and Programming, Version 4.6.5 (2013). http://www. gap-system.org.

[6] Golay, M.J.E.,Notes on digital coding, Proc. IRE, 37, 657. 1949.

[7] Grassl, Markus.Bounds on the minimum distance of linear codes and quantum codes. Online available at http://www.codetables.de. Acessado em 2011-2019.

[8] Jespers, E., del Río, A. Orders and Generic Constructions of Units, Walter de Gruyter GmbH Co KG, Nov 13, 2015 - Mathematics

[9] Jespers E., Leal G., Paques A.: Central idempotents in the rational group algebra of a finite nilpotent group. J. Algebra Appl. 2(1), 57-62 (2003).

[10] Jespers E., E.G. Goodaire and C. Polcino Milies, Alternative loop rings, Elsevier, Amsterdam, 1996 
[11] Jespers E., del Río Á., Olteanu G., Van Gelder I.: Group rings of finite strongly monomial groups: central units and primitive idempotents. J. Algebra 387, 99-116 (2013).

[12] Olteanu, G. ; Van Gelder, I. Construction of minimal non-Abelian left group codes. Des. Codes Cryptogr. (2015) 75:359-373

[13] C. Polcino Milies; Sehgal, S. K. An Introduction to Group Rings. Dordrecht: Kluwer Academic Publishers, (2002)

[14] Olivieri A., del Río Á., Simón J.: On monomial characters and central idempotents of rational group algebras. Commun. Algebra 32(4), 1531-1550 (2004).

[15] Olivieri, Aurora; del Río, Ángel An algorithm to compute the primitive central idempotents and the Wedderburn decomposition of a rational group algebra. J. Symbolic Comput. 35 (2003), no. 6, 673-687.

[16] Olteanu, G., Computing the Wedderburn decomposition of group algebras by the Brauer-Witt theorem, Math. Comp., 76 (258) (2007), 1073-1087 (electronic).

[17] Olteanu G.,Van Gelder I.: Finite group algebras of nilpotent groups: a complete set of orthogonal primitive idempotents. Finite Fields Appl. 17(2), 157-165 (2011).

[18] Sabin R., Lomonaco S.: Metacyclic error-correcting codes. Appl. Algebra Eng. Commun. Comput. 6(3), 191-210 (1995).

[19] SageMath, the Sage Mathematics Software System, The Sage Developers, 2019, https://www.sagemath.org.

[20] Shannon C. E. , A Mathematical Theory of Communication, The Bell System Technical Journal, 1948 